Supporting Information

\title{
Synthesis and Reactivity of the Rhenium Fulvene Sandwich Complex $\left[\operatorname{Re}\left(\eta^{6}-\mathrm{C}_{5} \mathrm{H}_{4} \mathrm{CH}_{2}\right)\left(\eta^{6}-\mathrm{C}_{6} \mathrm{H}_{6}\right)\right]^{+}$
}

\author{
Nina F. Suremann, Giuseppe Meola, Olivier Blacque, Henrik Braband and Roger Alberto* \\ Department of Chemistry, University of Zurich, Winterthurerstrasse 190, CH-8057 Zurich, Switzerland.
}

\section{Table of Content}

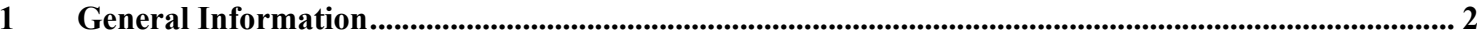

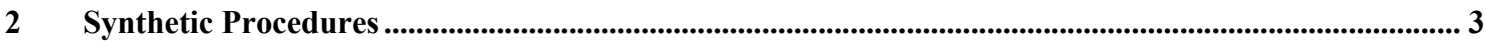

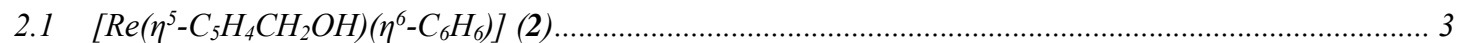

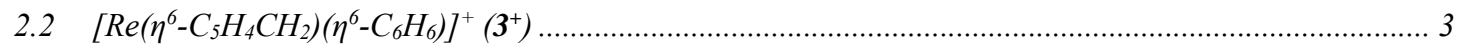

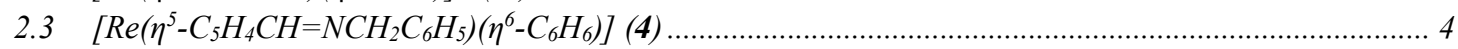

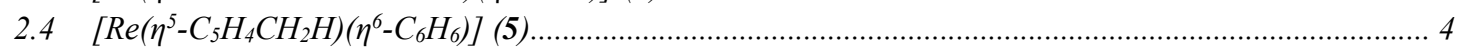

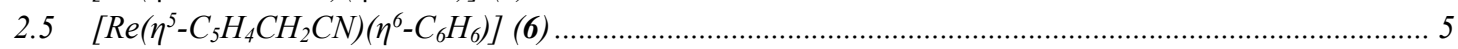

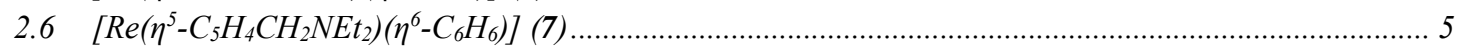

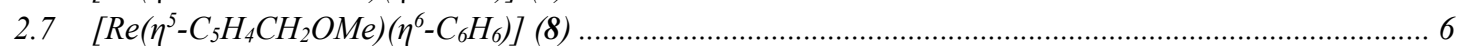

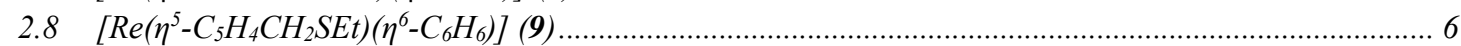

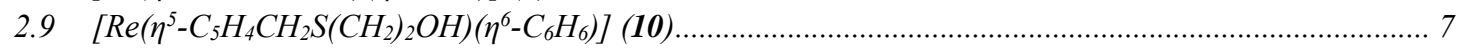

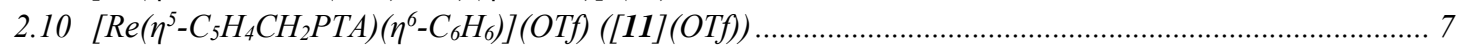

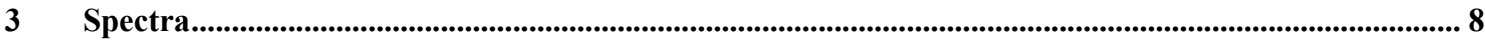

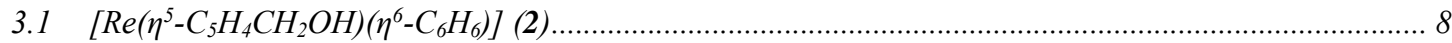

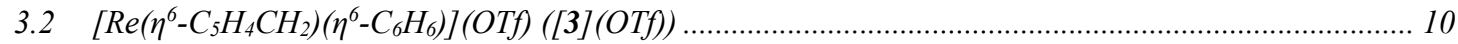

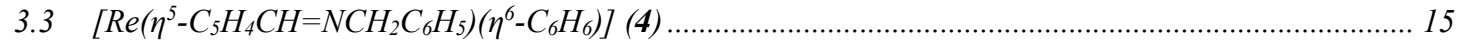

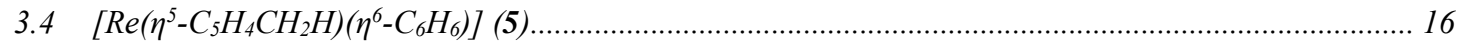

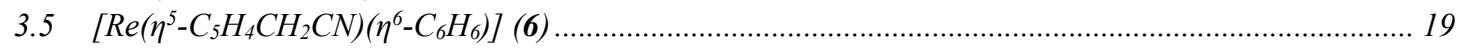

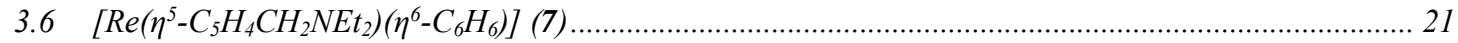

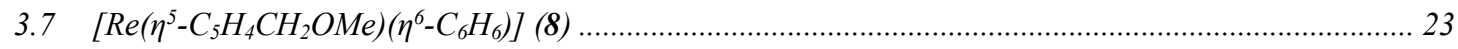

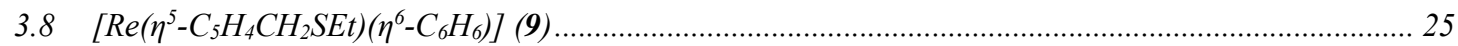

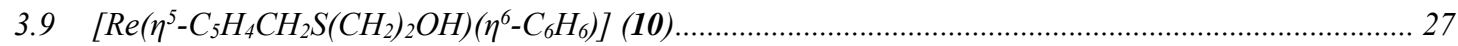

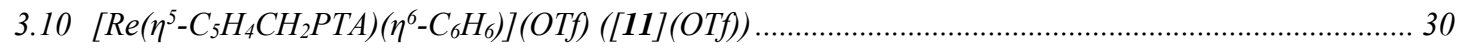

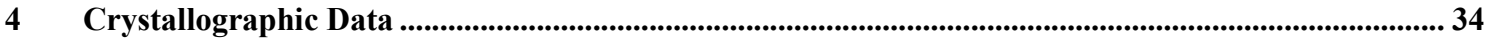

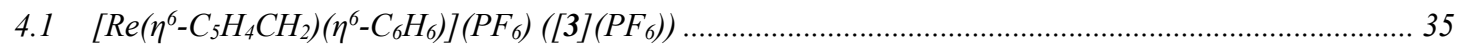

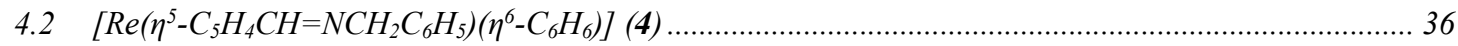

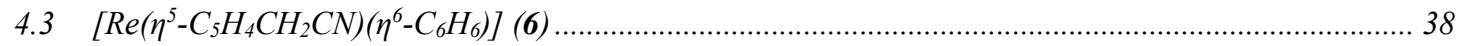

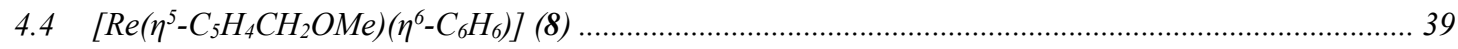

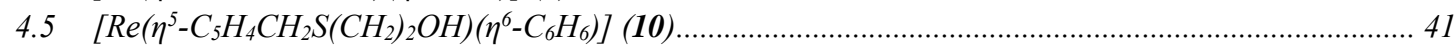

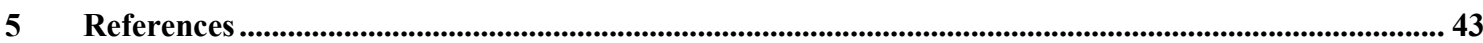




\section{General Information}

Materials: Unless otherwise stated, all chemicals were of reagent grade or higher, purchased from commercial sources and employed without further purification. Solvents for reactions were of p.a. grade and if indicated, THF and $\mathrm{Et}_{2} \mathrm{O}$ were dried over $\mathrm{Na}$ /benzophenone and $\mathrm{MeCN}$ was dried over $\mathrm{Al}_{2} \mathrm{O}_{3} ; \mathrm{H}_{2} \mathrm{O}$ was bidistilled. Reactions were carried out under $\mathrm{N}_{2}$ atmosphere handling standard Schlenk techniques in oven-dried $\left(115^{\circ} \mathrm{C}\right)$ glass equipment. Deuterated NMR solvents were purchased from Armar Chemicals or Cambridge Isotope Laboratories, Inc. (UK). Complex 1 was prepared according to literature known procedures starting from $\mathrm{Na}\left[\mathrm{ReO}_{4}\right] .{ }^{1,2} \mathbf{p H}$ : Merck indicator paper $\mathrm{pH} 1-14$ (universal indicator). NMR: NMR spectra were recorded in deuterated solvents at $298 \mathrm{~K}$ on Bruker AV-400 or Bruker AV500 spectrometers. Oxygen sensitive samples were measured in Young valve-closed NMR tubes. ${ }^{1} \mathrm{H}$ and ${ }^{13} \mathrm{C}$ chemical shifts $(\delta)$ are given in ppm relative to the first mentioned residual solvent resonances $\left(\mathrm{CD}_{3} \mathrm{CN}{ }^{1} \mathrm{H}: \delta 1.94,{ }^{13} \mathrm{C}: \delta 1.32,118.26 ; \mathrm{C}_{4} \mathrm{D}_{8} \mathrm{O}{ }^{1} \mathrm{H}: \delta 1.72,3.58,{ }^{13} \mathrm{C}: \delta 67.21,25.31 ; \mathrm{CDCl}_{3}{ }^{1} \mathrm{H}: \delta 7.26\right)$; coupling constants $(J)$ are given in hertz. Signal assignments are given according to arbitrary numbering depicted in the respective spectra and are based on coupling constants, increment calculations and/or supportive NMR experiments (HSQC, HMBC and ${ }^{13}$ C-DEPT). UPLC-ESI-MS: Waters Acquity UPLC System coupled to a Bruker Daltonics HCTTM ESI-MS, using an Acquity UPLC BEH C18 $1.7 \mu \mathrm{m}(2.1$ $\times 50 \mathrm{~mm}$ ) column. UPLC solvents were formic acid $(0.1 \%$ in millipore water) (solvent A) and MeCN UPLC grade (solvent B). Applied UPLC gradient: 0-0.5 min: 95\% A, 5\% B; 0.5-4.0 min: linear gradient from $95 \% \mathrm{~A}, 5 \% \mathrm{~B}$ to $0 \% \mathrm{~A}, 100 \% \mathrm{~B} ; 4.0-5.0 \mathrm{~min}: 0 \% \mathrm{~A}, 100 \% \mathrm{~B}$. The flow rate was $0.6 \mathrm{~mL}$ min-1. Detection was performed at 250 and $480 \mathrm{~nm}$ (DAD). IR: IR spectra were recorded on a SpectrumTwo FT-IR Spectrometer (Perkin-Elmer) equipped with a Specac Golden Gate ${ }^{T M}$ ATR (attenuated total reflection) accessory; applied as neat samples. High-resolution electrospray ionization mass spectrometry (HR-ESI-MS): HR-ESI-MS was performed on a QExactive (Thermo Fisher Scientific, Bremen, Germany) equipped with a heated ESI source connected to a Dionex Ultimate 3000 UPLC system. Samples were dissolved in $\mathrm{MeOH}$ or $\mathrm{MeCN}$ at ca. $50 \mu \mathrm{g} \mathrm{mL}^{-1}$; injection of $1 \mu \mathrm{L}$ on-flow with an XRS autosampler (CTC, Zwingen, Switzerland) (mobile phase: $\mathrm{MeOH}+0.1 \% \mathrm{HCOOH}$ or $\mathrm{CH}_{3} \mathrm{CN} / \mathrm{H}_{2} \mathrm{O}$ $(2: 8)+0.1 \% \mathrm{HCOOH}$; flow rate $\left.120 \mu \mathrm{L} \mathrm{mL}^{-1}\right)$; ion source parameters: spray voltage $3.0 \mathrm{kV}$, capillary temperature $280{ }^{\circ} \mathrm{C}$, sheath gas $30 \mathrm{~L} \mathrm{~min}^{-1}$, s-lens RF level 55.0; aux gas temperature $250{ }^{\circ} \mathrm{C}$; full scan MS in alternating $(+) /(-)$-ESI mode; mass ranges $80-1200$, 133-2000, or 200-3000 amu; resolution (full width half-maximum) 70000; automatic gain control (AGC) target $3.0010^{6}$; maximum allowed ion transfer time (IT) $30 \mathrm{~ms}$; mass calibration $<2 \mathrm{ppm}$ accuracy for $\mathrm{m} / z$ 130.06619-1621.96509 in (+)-ESI with Pierce ${ }^{\circledR}$ ESI calibration solutions (Thermo Fisher Scientific, Rockford, USA); lock masses: ubiquitous erucamide $(\mathrm{m} / \mathrm{z} 338.34174,(+)$-ESI). X-ray diffraction: Single crystal X-ray diffraction data were collected on a Rigaku OD Synergy (Pilatus 200K detector) diffractometer for [3]( $\left.\mathrm{PF}_{6}\right), \mathbf{8}$ and 10, and on a Rigaku OD Xcalibur (Ruby CCD detector) for $\mathbf{4}$ and 6, both equipped with an Oxford liquidnitrogen Cryostream cooler using a single wavelength $\mathrm{X}$-ray source from a micro-focus sealed $\mathrm{X}$-ray tube $\left(\mathrm{Cu} \mathrm{K} \mathrm{K}_{\alpha}\right.$ radiation $(\lambda=1.54184 \AA)$ for $[3]\left(\mathrm{PF}_{6}\right)$ and 8 or $\mathrm{Mo} \mathrm{K}_{\alpha}$ radiation $(\lambda=0.71073 \AA)$ for $\mathbf{4 ,} \mathbf{6}$ and 10). Suitable single crystals were manipulated into polybutene oil, selected and mounted on a flexible loop fixed on a goniometer head and transferred to the diffractometer. Pre-experiments, data collections, data reductions and analytical absorption corrections ${ }^{3}$ were performed with the program suite CrysAlis ${ }^{\text {Pro }}{ }^{4}$ Using Olex $2,{ }^{5}$ the structures were solved with the SHELXT small molecule structure solution program ${ }^{6}$ and refined with the SHELXL 2018/3 program package ${ }^{7}$ by full-matrix least-squares min-

imization on $\mathrm{F}^{2}$. PLATON was used to check the result of the X-ray analysis. For more details about the data collection and refinement parameters, see the CIF file and section 4 at the end of this supplementary information. 


\section{Synthetic Procedures}

\section{$2.1\left[\operatorname{Re}\left(\eta^{5}-\mathrm{C}_{5} \mathrm{H}_{4} \mathrm{CH}_{2} \mathrm{OH}\right)\left(\eta^{6}-\mathrm{C}_{6} \mathrm{H}_{6}\right)\right](2)$}

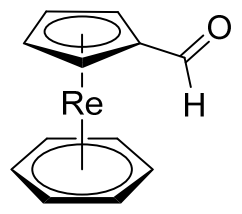

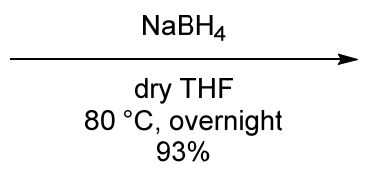

$93 \%$

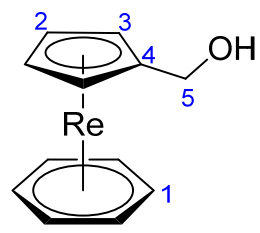

2

$\left[\boldsymbol{R e}\left(\boldsymbol{\eta}^{5}-\mathbf{C}_{5} \mathbf{H}_{4} \mathbf{C H}_{2} \mathbf{O H}\right)\left(\boldsymbol{\eta}^{6}-\mathbf{C}_{6} \mathbf{H}_{6}\right)\right]$ (2). Complex 1 (63 mg, $0.18 \mathrm{mmol}, 1.0$ eq.) and $\mathrm{NaBH}_{4}(69 \mathrm{mg}, 1.82 \mathrm{mmol}$, 10.3 eq.) were suspended in dry THF $(7 \mathrm{~mL})$. The orange reaction mixture was stirred overnight at $80{ }^{\circ} \mathrm{C}$. The slightly yellowish turbid mixture was concentrated and subsequently quenched with degassed $\mathrm{H}_{2} \mathrm{O}(5 \mathrm{~mL})$. The $\mathrm{THF}$ was evaporated and the product was extracted from the aqueous solution with $\mathrm{Et}_{2} \mathrm{O}(3 \times 3 \mathrm{~mL})$. After combining the organic phases, the solvent was evaporated to give $\mathbf{2}$ as analytically pure, colorless to yellowish solid. Yield: $59 \mathrm{mg}(93 \%)$.

Analysis. ${ }^{1} \mathrm{H}$ NMR (500 MHz, $\left.\mathrm{C}_{4} \mathrm{D}_{8} \mathrm{O}\right) \delta[\mathrm{ppm}]: 5.16\left(\mathrm{~s}, 2 \mathrm{H}, \mathrm{H}_{3}\right) ; 4.98\left(\mathrm{~s}, 2 \mathrm{H}, \mathrm{H}_{2}\right) ; 4.66\left(\mathrm{~s}, 6 \mathrm{H}, \mathrm{H}_{1}\right) ; 3.99(\mathrm{~d}$, $\left.J=5.95 \mathrm{~Hz}, 2 \mathrm{H}, \mathrm{H}_{5}\right) ; 2.89(\mathrm{t}, J=5.95 \mathrm{~Hz}, 1 \mathrm{H}, \mathrm{OH}) .{ }^{13} \mathrm{C} \mathrm{NMR}\left(125 \mathrm{MHz}, \mathrm{C}_{4} \mathrm{D}_{8} \mathrm{O}\right) \delta[\mathrm{ppm}]: 96.02\left(1 \mathrm{C}, \mathrm{C}_{4}\right) ; 73.49$ $\left(2 \mathrm{C}, \mathrm{C}_{3}\right) ; 71.92\left(2 \mathrm{C}, \mathrm{C}_{2}\right) ; 61.48\left(1 \mathrm{C}, \mathrm{C}_{5}\right) ; 60.98\left(6 \mathrm{C}, \mathrm{C}_{1}\right)$. IR (neat) $v\left[\mathrm{~cm}^{-1}\right]: 3357(\mathrm{w}), 3196(\mathrm{br} ., \mathrm{w}), 3086(\mathrm{w})$, 2958 (w), 2921 (s), 2851 (m), 1658 (w), 1632 (w), 1467 (w), 1419 (m), 1376 (w), 1260 (s), 1235 (w), 1093 (m), 1037 (s), 1019 (s), 969 (w), 904 (s), 814 (s), 800 (s). HR-ESI-MS m/z: [M] calcd for $\mathrm{C}_{12} \mathrm{H}_{13} \mathrm{ORe}, 360.05184$; found, 360.05136 .

\section{$2.2\left[\operatorname{Re}\left(\eta^{6}-\mathrm{C}_{5} \mathrm{H}_{4} \mathrm{CH}_{2}\right)\left(\eta^{6}-\mathrm{C}_{6} \mathrm{H}_{6}\right)\right]^{+}\left(3^{+}\right)$}

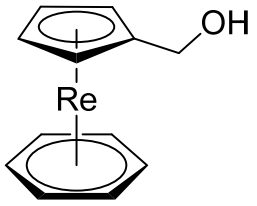

2

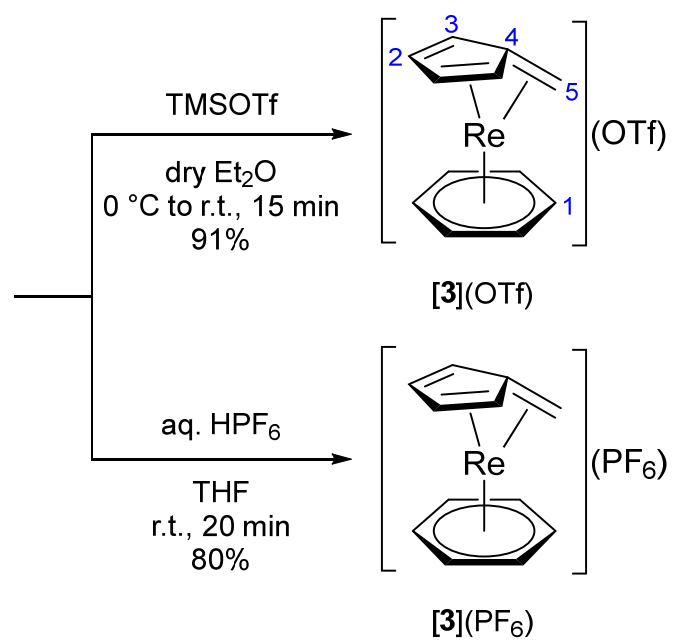

$\left[\boldsymbol{R e}\left(\eta^{6}-\mathbf{C}_{5} \mathbf{H}_{4} \mathbf{C H}_{2}\right)\left(\eta^{6}-\mathbf{C}_{6} \mathbf{H}_{6}\right)\right](\mathbf{O T f})([3](\mathbf{O T f}))$. The carbinol complex $\mathbf{2}$ (32 $\mathrm{mg}, 0.09 \mathrm{mmol}, 1.0$ eq.) was dissolved in dry $\mathrm{Et}_{2} \mathrm{O}(5 \mathrm{~mL})$. The slightly yellowish clear solution was cooled to $0{ }^{\circ} \mathrm{C}$ and a solution of trimethylsilyl trifluoromethanesulfonate (TMSOTf; $1 \%$ in $\mathrm{Et}_{2} \mathrm{O} ; 1.8 \mathrm{~mL}, 0.10 \mathrm{mmol}, 1.1$ eq.) was added dropwise. A precipitate formed instantaneously and the suspension was further stirred for $15 \mathrm{~min}$ at r.t. After decanting the supernatant, the solid residue was washed with $\mathrm{Et}_{2} \mathrm{O}(3 \times 3 \mathrm{~mL})$ and dried in vacuo to afford [3](OTf) as analytically pure, yellow solid. Yield: $40 \mathrm{mg}(91 \%)$. 


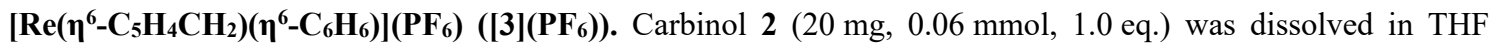
(3 mL). An aqueous solution of $\mathrm{HPF}_{6}(65 \% ; 1 \mathrm{~mL})$ was added dropwise to the yellow solution, which was then stirred for $20 \mathrm{~min}$ at r.t. Product $[3]\left(\mathrm{PF}_{6}\right)$ precipitated after $\mathrm{H}_{2} \mathrm{O}(5 \mathrm{~mL})$ was added and the remaining THF was evaporated. The aqueous supernatant was removed and the residue was washed with $\mathrm{H}_{2} \mathrm{O}(3 \times 3 \mathrm{~mL})$ and dried in vacuo. The product was recrystallized from $\mathrm{MeCN}$, yielding single crystals of [3] $\left(\mathrm{PF}_{6}\right)$, suitable for $\mathrm{X}$-ray diffraction analysis. Yield: $22 \mathrm{mg}(80 \%)$.

Analysis of [3](OTf). ${ }^{1} \mathrm{H}$ NMR (400 MHz, $\left.\mathrm{CD}_{3} \mathrm{CN}\right) \delta$ [ppm]: 6.03-6.01 (m, 2H, $\left.\mathrm{H}_{2}\right) ; 5.47$ (s, 6H, $\left.\mathrm{H}_{1}\right) ; 5.42-5.41$ $\left(\mathrm{m}, 2 \mathrm{H}, \mathrm{H}_{3}\right) ; 3.95\left(\mathrm{~s}, 2 \mathrm{H}, \mathrm{H}_{5}\right) .{ }^{1} \mathrm{H} \mathrm{NMR}\left(500 \mathrm{MHz}, \mathrm{C}_{4} \mathrm{D}_{8} \mathrm{O}\right) \delta[\mathrm{ppm}]: 6.32-6.30\left(\mathrm{~m}, 2 \mathrm{H}, \mathrm{H}_{2}\right) ; 5.66\left(\mathrm{~s}, 6 \mathrm{H}, \mathrm{H}_{1}\right) ; 5.58-$ $5.57\left(\mathrm{~m}, 2 \mathrm{H}, \mathrm{H}_{3}\right) ; 4.00\left(\mathrm{~s}, 2 \mathrm{H}, \mathrm{H}_{5}\right) .{ }^{13} \mathrm{C} \mathrm{NMR}\left(100 \mathrm{MHz}, \mathrm{CD}_{3} \mathrm{CN}\right) \delta[\mathrm{ppm}]: 97.20\left(1 \mathrm{C}, \mathrm{C}_{4}\right) ; 89.83\left(2 \mathrm{C}, \mathrm{C}_{2}\right) ; 83.42$ $\left(2 \mathrm{C}, \mathrm{C}_{3}\right) ; 80.63\left(6 \mathrm{C}, \mathrm{C}_{1}\right) ; 52.29\left(1 \mathrm{C}, \mathrm{C}_{5}\right) .{ }^{13} \mathrm{C} \mathrm{NMR}\left(125 \mathrm{MHz}, \mathrm{C}_{4} \mathrm{D}_{8} \mathrm{O}\right) \delta[\mathrm{ppm}]: 97.08\left(1 \mathrm{C}, \mathrm{C}_{4}\right) ; 90.41\left(2 \mathrm{C}, \mathrm{C}_{2}\right)$; $83.27\left(2 \mathrm{C}, \mathrm{C}_{3}\right) ; 80.75\left(6 \mathrm{C}, \mathrm{C}_{1}\right) ; 51.50\left(1 \mathrm{C}, \mathrm{C}_{5}\right)$. UPLC-ESI-MS: $m / z=343.1[\mathrm{M}]^{+}$. IR (neat) $v\left[\mathrm{~cm}^{-1}\right]: 3094(\mathrm{w})$, 1426 (w), 1408 (w), 1259 (s), 1222 (m), 1152 (s), 1029 (s), 970 (w), 895 (w), 839 (m), 754 (w). HR-ESI-MS m/z: $[\mathrm{M}]^{+}$calcd for $\mathrm{C}_{12} \mathrm{H}_{12} \mathrm{Re}, 343.04911$; found, 343.04940.

\section{$2.3 \quad\left[\operatorname{Re}\left(\eta^{5}-\mathrm{C}_{5} \mathrm{H}_{4} \mathrm{CH}=\mathrm{NCH}_{2} \mathrm{C}_{6} \mathrm{H}_{5}\right)\left(\eta^{6}-\mathrm{C}_{6} \mathrm{H}_{6}\right)\right](4)$}

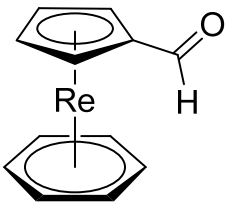

1

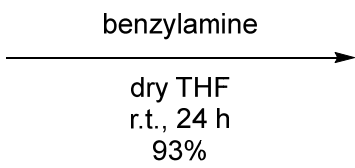

$93 \%$

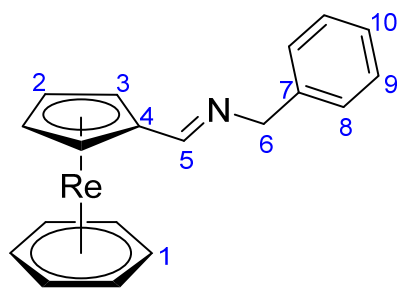

4

$\left[\boldsymbol{R e}\left(\boldsymbol{\eta}^{5}-\mathbf{C}_{5} \mathbf{H}_{4} \mathbf{C H N C H} \mathbf{C}_{6} \mathbf{H}_{5}\right)\left(\boldsymbol{\eta}^{6}-\mathbf{C}_{6} \mathbf{H}_{6}\right)\right](\mathbf{4})$. Benzylamine $(61 \mu \mathrm{L}, 0.56 \mathrm{mmol}, 10$ eq. $)$ was added to a yellow solution of complex 1 (20 mg, $0.06 \mathrm{mmol}, 1.0 \mathrm{eq}$.) in dry THF (3 mL). The reaction mixture was stirred for $24 \mathrm{~h}$ at r.t. The remaining solvent was evaporated in vacuo and the residue was suspended and washed with degassed $\mathrm{H}_{2} \mathrm{O}$ $(3 \times 2 \mathrm{~mL})$ and cold dry pentane $(2 \times 1 \mathrm{~mL})$. The product was recrystallized from THF, yielding single crystals of 4, suitable for X-ray diffraction analysis. Yield: $23 \mathrm{mg}(93 \%)$.

Analysis. ${ }^{1} \mathrm{H}$ NMR (500 MHz, $\left.\mathrm{C}_{4} \mathrm{D}_{8} \mathrm{O}\right) \delta[\mathrm{ppm}]: 8.04\left(\mathrm{~s}, 1 \mathrm{H}, \mathrm{H}_{5}\right) ; 7.26-7.23\left(\mathrm{~m}, 2 \mathrm{H}, \mathrm{CH}_{\text {arom }}\right) ; 7.19-7.16(\mathrm{~m}, 3 \mathrm{H}$, $\mathrm{CH}_{\text {arom }}$ ); 5.50-5.49 (m, 2H, $\left.\mathrm{H}_{\mathrm{Cp}}\right) ; 5.15-5.14\left(\mathrm{~m}, 2 \mathrm{H}, \mathrm{H}_{\mathrm{Cp}}\right) ; 4.69$ (s, 6H, $\left.\mathrm{H}_{1}\right) ; 4.49$ (s, 2H, $\left.\mathrm{H}_{6}\right)$. UPLC-ESI-MS: $m / z=448.1[\mathrm{M}+\mathrm{H}]^{+}$. IR (neat) $v\left[\mathrm{~cm}^{-1}\right]: 3059(\mathrm{w}), 2828(\mathrm{w}), 1637(\mathrm{~s}), 1495(\mathrm{~m}), 1453(\mathrm{~m}), 1419(\mathrm{~m}), 1367(\mathrm{~m})$, $1333(\mathrm{w}), 1246(\mathrm{~m}), 1160(\mathrm{w}), 1042(\mathrm{w}), 1022$ (w), $981(\mathrm{~m}), 969(\mathrm{~m}), 898(\mathrm{w}), 816$ (s), 764 (m), 733 (s). HR-ESIMS $m / z:[\mathrm{M}+\mathrm{H}]^{+}$calcd for $\mathrm{C}_{19} \mathrm{H}_{18} \mathrm{NRe}, 448.1069$; found, 448.1060 .

\section{$2.4\left[\operatorname{Re}\left(\eta^{5}-\mathrm{C}_{5} \mathrm{H}_{4} \mathrm{CH}_{2} \mathrm{H}\right)\left(\eta^{6}-\mathrm{C}_{6} \mathrm{H}_{6}\right)\right](5)$}

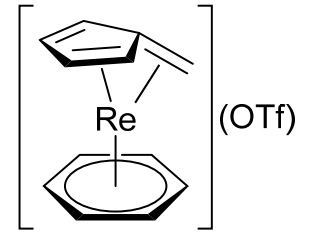

$[3](\mathrm{OTf})$

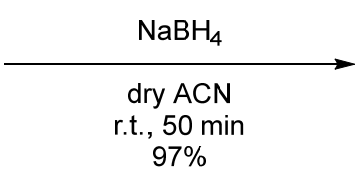

$97 \%$

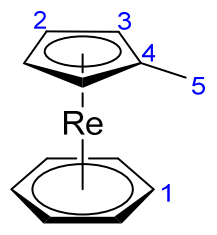

5

$\left[\boldsymbol{R e}\left(\boldsymbol{\eta}^{5}-\mathbf{C}_{5} \mathbf{H}_{4} \mathbf{C H}_{2} \mathbf{H}\right)\left(\boldsymbol{\eta}^{6}-\mathbf{C}_{6} \mathbf{H}_{6}\right)\right]$ (5). The compound [3](OTf) (16 mg, $0.03 \mathrm{mmol}, 1.0$ eq.) and $\mathrm{NaBH}_{4}(13 \mathrm{mg}$, $0.34 \mathrm{mmol}, 10.4$ eq.) were suspended in dry $\mathrm{MeCN}(1.5 \mathrm{~mL})$. The reaction mixture was stirred for $50 \mathrm{~min}$ at r.t. 
After quenching the unreacted $\mathrm{NaBH}_{4}$ with degassed $\mathrm{H}_{2} \mathrm{O}(3.5 \mathrm{~mL})$, the $\mathrm{MeCN}$ was evaporated and the product was extracted with $\mathrm{Et}_{2} \mathrm{O}(3 \times 2 \mathrm{~mL})$ from the aqueous solution. After combining the organic phases, the solvent was evaporated to give 5 as analytically pure, light yellow solid. Yield: $11 \mathrm{mg}$ (97\%).

Analysis. ${ }^{1} \mathrm{H}$ NMR (500 MHz, $\left.\mathrm{C}_{4} \mathrm{D}_{8} \mathrm{O}\right) \delta[\mathrm{ppm}]:$ 5.08-5.07 (m, 2H, $\left.\mathrm{H}_{3}\right) ; 4.92-4.91\left(\mathrm{~m}, 2 \mathrm{H}, \mathrm{H}_{2}\right) ; 4.59\left(\mathrm{~s}, 6 \mathrm{H}, \mathrm{H}_{1}\right)$; $2.02\left(\mathrm{~s}, 2 \mathrm{H}, \mathrm{H}_{5}\right) .{ }^{13} \mathrm{C}$ NMR $\left(125 \mathrm{MHz}, \mathrm{C}_{4} \mathrm{D}_{8} \mathrm{O}\right) \delta[\mathrm{ppm}]: 89.31\left(1 \mathrm{C}, \mathrm{C}_{4}\right) ; 75.21\left(2 \mathrm{C}, \mathrm{C}_{3}\right) ; 71.60\left(2 \mathrm{C}, \mathrm{C}_{2}\right) ; 60.87(6 \mathrm{C}$, $\left.\mathrm{C}_{1}\right) ; 16.17\left(1 \mathrm{C}, \mathrm{C}_{5}\right)$. UPLC-ESI-MS: $m / z=345.1[\mathrm{M}+\mathrm{H}]^{+}$. IR (neat) $v\left[\mathrm{~cm}^{-1}\right]: 3359(\mathrm{w}), 3188(\mathrm{w}), 3097(\mathrm{w}), 2960$ (m), 2922 (s), 2852 (m), 1658 (w), 1632 (m), 1467 (w), 1421 (w), 1261 (s), 1093 (s), 1020 (s), 907 (w), 799 (s). HR-ESI-MS $m / z$ : [M] $]^{+}$calcd for $\mathrm{C}_{12} \mathrm{H}_{13} \mathrm{Re}, 344.05693$; found, 344.05692 .

\section{$2.5 \quad\left[\operatorname{Re}\left(\eta^{5}-\mathrm{C}_{5} \mathrm{H}_{4} \mathrm{CH}_{2} \mathrm{CN}\right)\left(\eta^{6}-\mathrm{C}_{6} \mathrm{H}_{6}\right)\right](6)$}

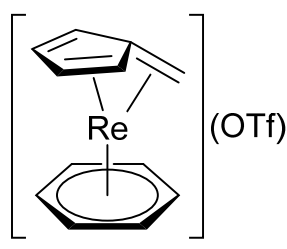

[3](OTf)

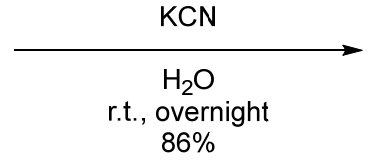

$86 \%$

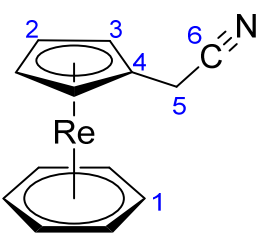

6

$\left[\boldsymbol{R e}\left(\boldsymbol{\eta}^{5}-\mathbf{C}_{5} \mathbf{H}_{4} \mathbf{C H}_{2} \mathbf{C N}\right)\left(\boldsymbol{\eta}^{6}-\mathbf{C}_{6} \mathbf{H}_{6}\right)\right]$ (6). The compound [3](OTf) (11 mg, $0.02 \mathrm{mmol}, 1.0$ eq.) and $\mathrm{KCN}(12 \mathrm{mg}$, $0.18 \mathrm{mmol}, 8.4$ eq. ) were dissolved in degassed $\mathrm{H}_{2} \mathrm{O}(0.8 \mathrm{~mL})$. The yellow clear reaction mixture was stirred overnight at r.t. to form a colorless precipitate in a still slightly yellowish solution. The product was extracted with $\mathrm{Et}_{2} \mathrm{O}(3 \times 2 \mathrm{~mL})$ from the aqueous solution, $\mathrm{Et}_{2} \mathrm{O}$ was evaporated and the yellowish solid was dried in vacuo to result in analytically pure $\mathbf{6}$ as colorless solid. The product was recrystallized from $\mathrm{Et}_{2} \mathrm{O}$, yielding single crystals of 6, suitable for X-ray diffraction analysis. Yield: $7.0 \mathrm{mg}(86 \%)$.

Analysis. ${ }^{1} \mathrm{H}$ NMR $\left(500 \mathrm{MHz}, \mathrm{C}_{4} \mathrm{D}_{8} \mathrm{O}\right) \delta[\mathrm{ppm}]: 5.23\left(\mathrm{~s}, 2 \mathrm{H}, \mathrm{H}_{3}\right) ; 5.01\left(\mathrm{~s}, 2 \mathrm{H}, \mathrm{H}_{2}\right) ; 4.73\left(\mathrm{~s}, 6 \mathrm{H}, \mathrm{H}_{1}\right) ; 3.51(\mathrm{~s}, 2 \mathrm{H}$, $\left.\mathrm{H}_{5}\right) .{ }^{13} \mathrm{C}$ NMR $\left(125 \mathrm{MHz}, \mathrm{C}_{4} \mathrm{D}_{8} \mathrm{O}\right) \delta[\mathrm{ppm}]: 118.40\left(1 \mathrm{C}, \mathrm{C}_{6}\right) ; 83.48\left(1 \mathrm{C}, \mathrm{C}_{4}\right) ; 73.38\left(2 \mathrm{C}, \mathrm{C}_{3}\right) ; 72.05\left(2 \mathrm{C}, \mathrm{C}_{2}\right) ; 62.11$ $\left(6 \mathrm{C}, \mathrm{C}_{1}\right) ; 20.06\left(1 \mathrm{C}, \mathrm{C}_{5}\right)$. UPLC-ESI-MS: $\mathrm{m} / z=370.0[\mathrm{M}+\mathrm{H}]^{+}$. IR (neat) $v\left[\mathrm{~cm}^{-1}\right]: 3358(\mathrm{w}), 3185(\mathrm{w}), 3059(\mathrm{w})$, 2961 (m), 2922 (m), 2852 (m), 2255 (w, CN), 1659 (w), 1632 (w), 1467 (m), 1414 (w), 1261 (s), 1094 (m), 1021 (s), 798 (s), 706 (m). HR-ESI-MS m/z: [M] calcd for $\mathrm{C}_{13} \mathrm{H}_{12} \mathrm{NRe}, 369.05218$; found, 369.05276.

\section{$2.6\left[\operatorname{Re}\left(\eta^{5}-\mathrm{C}_{5} \mathrm{H}_{4} \mathrm{CH}_{2} \mathrm{NEt}_{2}\right)\left(\eta^{6}-\mathrm{C}_{6} \mathrm{H}_{6}\right)\right](7)$}

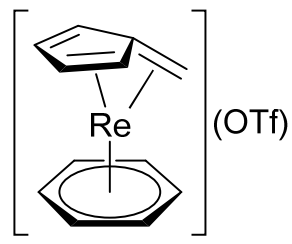

[3](OTf)

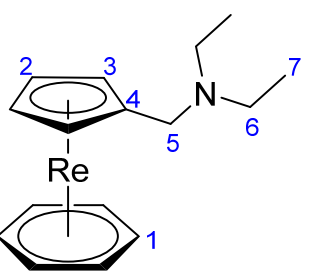

7

$\left[\boldsymbol{R e}\left(\boldsymbol{\eta}^{5}-\mathbf{C}_{5} \mathbf{H}_{4} \mathbf{C H}_{2} \mathbf{N E t}_{2}\right)\left(\boldsymbol{\eta}^{6}-\mathbf{C}_{6} \mathbf{H}_{6}\right)\right]$ (7). The complex [3](OTf) (10 mg, $0.02 \mathrm{mmol}, 1.0$ eq.) was dissolved in degassed diethylamine $(1 \mathrm{~mL}, 9.71 \mathrm{mmol}, 441 \mathrm{eq}$.). The reaction mixture was stirred for $25 \mathrm{~min}$ at r.t. before the remaining diethylamine was evaporated and the product was extracted with pentane $(3 \times 1 \mathrm{~mL})$ from a brownyellow sticky residue. After combining the organic phases, the solvent was evaporated to give 7 as analytically pure, yellow-brownish oil. Yield: $9 \mathrm{mg}(95 \%)$. 
Analysis. ${ }^{1} \mathrm{H}$ NMR (400 MHz, $\left.\mathrm{C}_{4} \mathrm{D}_{8} \mathrm{O}\right) \delta[\mathrm{ppm}]$ : 5.13-5.11 (m, 2H, $\left.\mathrm{H}_{3}\right) ; 4.98-4.96\left(\mathrm{~m}, 2 \mathrm{H}, \mathrm{H}_{2}\right) ; 4.62\left(\mathrm{~s}, 6 \mathrm{H}, \mathrm{H}_{1}\right)$; $3.21\left(\mathrm{~s}, 2 \mathrm{H}, \mathrm{H}_{5}\right) ; 2.42\left(\mathrm{q}, J=7.12 \mathrm{~Hz}, 4 \mathrm{H}, \mathrm{H}_{6}\right) ; 0.96\left(\mathrm{t}, J=7.12 \mathrm{~Hz}, 6 \mathrm{H}, \mathrm{H}_{7}\right) .{ }^{13} \mathrm{C} \mathrm{NMR}\left(100 \mathrm{MHz}, \mathrm{C}_{4} \mathrm{D}_{8} \mathrm{O}\right) \delta$ [ppm]: $90.24\left(1 \mathrm{C}, \mathrm{C}_{4}\right) ; 75.53\left(2 \mathrm{C}, \mathrm{C}_{3}\right) ; 72.00\left(2 \mathrm{C}, \mathrm{C}_{2}\right) ; 60.94\left(6 \mathrm{C}, \mathrm{C}_{1}\right) ; 54.34\left(1 \mathrm{C}, \mathrm{C}_{5}\right) ; 46.93\left(2 \mathrm{C}, \mathrm{C}_{6}\right) ; 12.65(2 \mathrm{C}$, $\mathrm{C}_{7}$ ). ${ }^{15} \mathrm{~N}$ NMR $\left(40 \mathrm{MHz}, \mathrm{C}_{4} \mathrm{D}_{8} \mathrm{O}\right) \delta[\mathrm{ppm}]:$ 7.71. UPLC-ESI-MS: $m / z=415.1[\mathrm{M}]^{+}$.

\section{$2.7 \quad\left[\operatorname{Re}\left(\eta^{5}-\mathrm{C}_{5} \mathrm{H}_{4} \mathrm{CH}_{2} \mathrm{OMe}\right)\left(\eta^{6}-\mathrm{C}_{6} \mathrm{H}_{6}\right)\right](8)$}

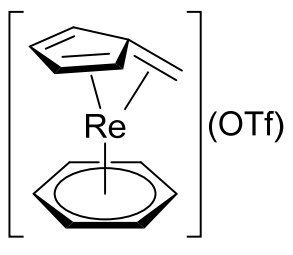

[3](OTf)

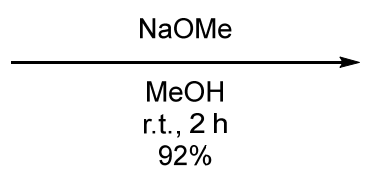

$92 \%$

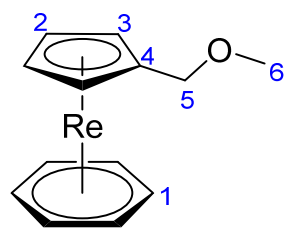

8

$\left[\boldsymbol{R e}\left(\boldsymbol{\eta}^{5}-\mathbf{C}_{5} \mathbf{H}_{4} \mathbf{C H}_{2} \mathrm{OMe}\right)\left(\boldsymbol{\eta}^{6}-\mathbf{C}_{6} \mathbf{H}_{6}\right)\right] \mathbf{( 8 )}$. The complex [3](OTf) (6 mg, $0.01 \mathrm{mmol}, 1.0$ eq.) and $\mathrm{NaOMe}(6 \mathrm{mg}$, $0.11 \mathrm{mmol}, 9.3 \mathrm{eq}$.) were dissolved in degassed $\mathrm{MeOH}(0.5 \mathrm{~mL})$. The yellow turbid reaction mixture was stirred for $2 \mathrm{~h}$ at r.t. and turned colorless. The remaining $\mathrm{MeOH}$ was evaporated and the product was extracted from the colorless-yellowish solid residue with degassed pentane $(3 \times 1 \mathrm{~mL})$ to give $\mathbf{8}$ as analytically pure, yellowish solid. The product was recrystallized from pentane, yielding single crystals of $\mathbf{8}$, suitable for X-ray diffraction analysis. Yield: $4 \mathrm{mg}(92 \%)$.

Analysis. ${ }^{1} \mathrm{H}$ NMR $\left(500 \mathrm{MHz}, \mathrm{C}_{4} \mathrm{D}_{8} \mathrm{O}\right) \delta[\mathrm{ppm}]: 5.16-5.14\left(\mathrm{~m}, 2 \mathrm{H}, \mathrm{H}_{3}\right) ; 5.01-4.99\left(\mathrm{~m}, 2 \mathrm{H}, \mathrm{H}_{2}\right) ; 4.64\left(\mathrm{~s}, 6 \mathrm{H}, \mathrm{H}_{1}\right)$; $3.90\left(\mathrm{~s}, 2 \mathrm{H}, \mathrm{H}_{5}\right) ; 3.20\left(\mathrm{~s}, 3 \mathrm{H}, \mathrm{H}_{6}\right) .{ }^{13} \mathrm{C} \mathrm{NMR}\left(125 \mathrm{MHz}, \mathrm{C}_{4} \mathrm{D}_{8} \mathrm{O}\right) \delta[\mathrm{ppm}]: 89.62\left(1 \mathrm{C}, \mathrm{C}_{4}\right) ; 74.73\left(2 \mathrm{C}, \mathrm{C}_{3}\right) ; 72.65$ $\left(1 \mathrm{C}, \mathrm{C}_{5}\right) ; 72.44\left(2 \mathrm{C}, \mathrm{C}_{2}\right) ; 61.11\left(6 \mathrm{C}, \mathrm{C}_{1}\right) ; 57.14\left(1 \mathrm{C}, \mathrm{C}_{6}\right)$. UPLC-ESI-MS: $m / z=375.5[\mathrm{M}+\mathrm{H}]^{+} . \mathrm{IR}(\mathrm{neat}) v\left[\mathrm{~cm}^{-1}\right]$ : 2954 (m), 2922 (s), 2852 (m), 1460 (w), 1376 (w), 1261 (m), $1093(\mathrm{~m}), 1020$ (m), 801 (m). HR-ESI-MS m/z: [M] calcd for $\mathrm{C}_{13} \mathrm{H}_{15} \mathrm{ORe}, 374.06749$; found, 374.06754 .

\section{$2.8 \quad\left[\operatorname{Re}\left(\eta^{5}-\mathrm{C}_{5} \mathrm{H}_{4} \mathrm{CH}_{2} \mathrm{SEt}\right)\left(\eta^{6}-\mathrm{C}_{6} \mathrm{H}_{6}\right)\right](9)$}

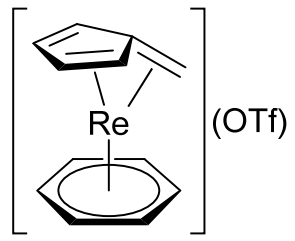

[3](OTf)

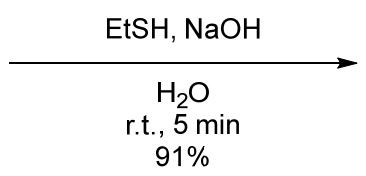

$91 \%$

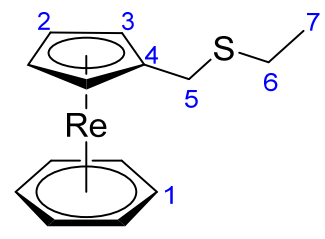

9

$\left[\boldsymbol{R e}\left(\boldsymbol{\eta}^{5}-\mathbf{C}_{5} \mathbf{H}_{4} \mathbf{C H}_{2} \mathbf{S E t}\right)\left(\boldsymbol{\eta}^{6}-\mathbf{C}_{6} \mathbf{H}_{6}\right)\right]$ (9). EtSH $(0.3 \mathrm{~mL}, 4.06 \mathrm{mmol}, 184.5$ eq. $)$ was added to a solution of $\mathrm{NaOH}$ (188 mg, $4.70 \mathrm{mmol}, 213.6$ eq.) in $\mathrm{H}_{2} \mathrm{O}(1.5 \mathrm{~mL})$ and mixed thoroughly. A yellow clear solution of [3](OTf) (11 mg, $0.02 \mathrm{mmol}, 1.0$ eq.) in $\mathrm{H}_{2} \mathrm{O}(1 \mathrm{~mL})$ was added dropwise to the alkaline EtSH solution at r.t., resulting in a turbid colorless mixture. After $5 \mathrm{~min}$, the product was extracted from the aqueous mixture with $\mathrm{Et}_{2} \mathrm{O}(3 \times 3 \mathrm{~mL})$, the solvent was evaporated and the colorless solid was dried in vacuo to give $\mathbf{9}$ in analytical pure quality. Yield: $8 \mathrm{mg}(91 \%)$.

Analysis. ${ }^{1} \mathrm{H}$ NMR $\left(400 \mathrm{MHz}, \mathrm{C}_{4} \mathrm{D}_{8} \mathrm{O}\right) \delta[\mathrm{ppm}]: 5.17-5.15\left(\mathrm{~m}, 2 \mathrm{H}, \mathrm{H}_{3}\right) ; 4.97-4.96\left(\mathrm{~m}, 2 \mathrm{H}, \mathrm{H}_{2}\right) ; 4.65\left(\mathrm{~s}, 6 \mathrm{H}, \mathrm{H}_{1}\right)$; $3.36\left(\mathrm{~s}, 2 \mathrm{H}, \mathrm{H}_{5}\right) ; 2.47$ (q, $\left.J=7.36 \mathrm{~Hz}, 2 \mathrm{H}, \mathrm{H}_{6}\right) ; 1.18\left(\mathrm{t}, J=7.38 \mathrm{~Hz}, 3 \mathrm{H}_{,} \mathrm{H}_{7}\right) .{ }^{13} \mathrm{C} \mathrm{NMR}\left(100 \mathrm{MHz}, \mathrm{C}_{4} \mathrm{D}_{8} \mathrm{O}\right) \delta$ [ppm]: $91.10\left(1 \mathrm{C}, \mathrm{C}_{4}\right) ; 74.28\left(2 \mathrm{C}, \mathrm{C}_{3}\right) ; 71.91\left(2 \mathrm{C}, \mathrm{C}_{2}\right) ; 61.39\left(6 \mathrm{C}, \mathrm{C}_{1}\right) ; 33.37\left(1 \mathrm{C}, \mathrm{C}_{5}\right) ; 26.33\left(1 \mathrm{C}, \mathrm{C}_{6}\right) ; 14.85(1 \mathrm{C}$, $\mathrm{C}_{7}$ ). UPLC-ESI-MS: $m / z=403.1[\mathrm{M}-\mathrm{H}]^{+}$. IR (neat) $v\left[\mathrm{~cm}^{-1}\right]$ : $3355(\mathrm{w}), 3183(\mathrm{w}), 3054(\mathrm{w}), 2962(\mathrm{w}), 2922(\mathrm{~m})$, 2852 (w), 1658 (w), 1632 (w), 1451 (w), 1418 (m), 1259 (m), 1233 (m), 1092 (m), 1021 (m), 987 (m), 969 (m), 908 (w), 812 (s), 718 (w). HR-ESI-MS m/z: [M] calcd for $\mathrm{C}_{14} \mathrm{H}_{17} \mathrm{ReS}, 404.06030$; found, 404.06035. 


\section{$2.9\left[\operatorname{Re}\left(\eta^{5}-\mathrm{C}_{5} \mathrm{H}_{4} \mathrm{CH}_{2} \mathrm{~S}\left(\mathrm{CH}_{2}\right)_{2} \mathrm{OH}\right)\left(\eta^{6}-\mathrm{C}_{6} \mathrm{H}_{6}\right)\right](10)$}

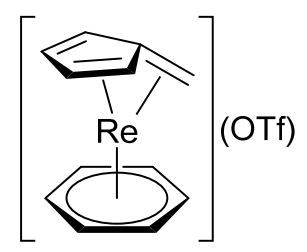

[3](OTf)

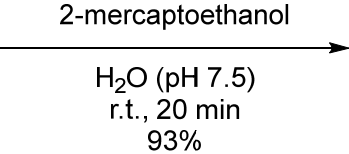

$93 \%$

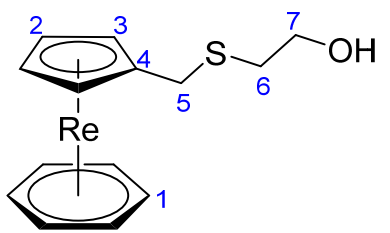

10

$\left[\operatorname{Re}\left(\eta^{5}-\mathbf{C}_{5} \mathbf{H}_{4} \mathbf{C H}_{2} \mathbf{S C H}_{2} \mathbf{C H}_{2} \mathbf{O H}\right)\left(\eta^{6}-\mathbf{C}_{6} \mathbf{H}_{6}\right)\right]$ (10). The complex [3](OTf) (20 mg, $0.04 \mathrm{mmol}, 1.0$ eq.) was dissolved in degassed $\mathrm{H}_{2} \mathrm{O}(3 \mathrm{~mL})$. The $\mathrm{pH}$ of the solution was adjusted to $\mathrm{pH} 7.5$ with a $2 \mathrm{M} \mathrm{Na}_{2} \mathrm{CO}_{3}$ solution. 2Mercaptoethanol ( $14 \mu \mathrm{L}, 0.21 \mathrm{mmol}, 5.0 \mathrm{eq}$.) was added to the yellow solution and the reaction mixture was stirred for $20 \mathrm{~min}$ at r.t. to form a colorless precipitate. The supernatant was removed and the residue was washed with degassed $\mathrm{H}_{2} \mathrm{O}(3 \times 3 \mathrm{~mL})$ and dried in vacuo. The product was recrystallized from $\mathrm{Et}_{2} \mathrm{O}$, yielding single crystals of 10, suitable for X-ray diffraction analysis. Yield: $16 \mathrm{mg}(93 \%)$.

Analysis. ${ }^{1} \mathrm{H}$ NMR $\left(500 \mathrm{MHz}, \mathrm{C}_{4} \mathrm{D}_{8} \mathrm{O}\right) \delta[\mathrm{ppm}]: 5.19-5.17\left(\mathrm{~m}, 2 \mathrm{H}, \mathrm{H}_{3}\right) ; 4.98-4.96\left(\mathrm{~m}, 2 \mathrm{H}, \mathrm{H}_{2}\right) ; 4.65\left(\mathrm{~s}, 6 \mathrm{H}, \mathrm{H}_{1}\right)$; $3.68(\mathrm{t}, J=5.73 \mathrm{~Hz}, 1 \mathrm{H}, \mathrm{OH}) ; 3.57$ (q, hidden behind solvent signal, $\left.J=6.29 \mathrm{~Hz}, 2 \mathrm{H}, \mathrm{H}_{7}\right) ; 3.40\left(\mathrm{~s}, 2 \mathrm{H}, \mathrm{H}_{5}\right) ; 2.57$ $\left(\mathrm{t}, J=7.03 \mathrm{~Hz}, 2 \mathrm{H}, \mathrm{H}_{6}\right) .{ }^{13} \mathrm{C}$ NMR $\left(125 \mathrm{MHz}, \mathrm{C}_{4} \mathrm{D}_{8} \mathrm{O}\right) \delta[\mathrm{ppm}]: 91.11\left(1 \mathrm{C}, \mathrm{C}_{4}\right) ; 74.32\left(2 \mathrm{C}, \mathrm{C}_{3}\right) ; 71.94\left(2 \mathrm{C}, \mathrm{C}_{2}\right)$; $62.42\left(1 \mathrm{C}, \mathrm{C}_{7}\right) ; 61.40\left(6 \mathrm{C}, \mathrm{C}_{1}\right) ; 35.35\left(1 \mathrm{C}, \mathrm{C}_{6}\right) ; 33.93\left(1 \mathrm{C}, \mathrm{C}_{5}\right)$. UPLC-ESI-MS: $m / z=421.1[\mathrm{M}+\mathrm{H}]^{+}$. IR (neat) $v$

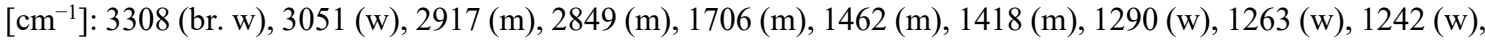
$1123(\mathrm{w}), 1045$ (m), 1034 (m), $1021(\mathrm{~m}), 988(\mathrm{~m}), 970(\mathrm{~m}), 814(\mathrm{~s})$.

\section{$2.10\left[\operatorname{Re}\left(\eta^{5}-\mathrm{C}_{5} \mathrm{H}_{4} \mathrm{CH}_{2} \mathrm{PTA}\right)\left(\eta^{6}-\mathrm{C}_{6} \mathrm{H}_{6}\right)\right](\mathrm{OTf})([11](\mathrm{OTf}))$}

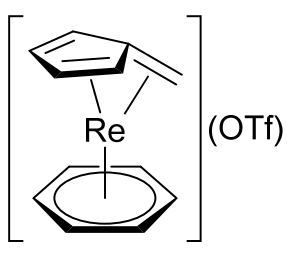

[3](OTf)

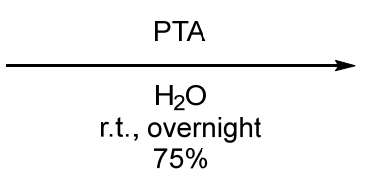

$75 \%$

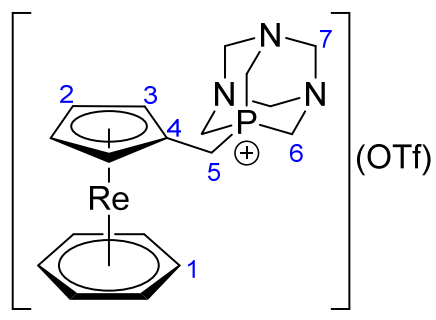

$[11](\mathrm{OTf})$

$\left[\mathbf{R e}\left(\boldsymbol{\eta}^{5}-\mathbf{C}_{5} \mathbf{H}_{4} \mathbf{C H}_{2} \mathbf{P T A}\right)\left(\eta^{6}-\mathbf{C}_{6} \mathbf{H}_{6}\right)\right](\mathbf{O T f})([11](\mathbf{O T f}))$. The complex [3](OTf) $(10 \mathrm{mg}, 0.02 \mathrm{mmol}, 1.0$ eq. $)$ and 1,3,5-triaza-7-phosphaadamantane (PTA) $\left(5 \mathrm{mg}, 0.03 \mathrm{mmol}, 1.6\right.$ eq.) were dissolved in degassed $\mathrm{H}_{2} \mathrm{O}(1.5 \mathrm{~mL})$. The clear yellow reaction mixture was stirred overnight at r.t. to form a brownish-colorless precipitate in a slightly yellowish solution. The supernatant was removed and the almost colorless solid residue was washed with $\mathrm{H}_{2} \mathrm{O}$ $(3 \times 1 \mathrm{~mL})$ and dried in vacuo to afford analytically pure [11](OTf) as colorless solid. Yield: $10 \mathrm{mg}(75 \%)$.

Analysis. ${ }^{1} \mathrm{H}$ NMR (400 MHz, $\left.\mathrm{CD}_{3} \mathrm{CN}\right) \delta[\mathrm{ppm}]:$ 5.17-5.16 (m, 2H, $\left.\mathrm{H}_{3}\right) ; 5.12-5.11\left(\mathrm{~m}, 2 \mathrm{H}, \mathrm{H}_{2}\right) ; 4.75\left(\mathrm{~s}, 6 \mathrm{H}, \mathrm{H}_{1}\right)$; 4.48-4.34 (m, $\left.12 \mathrm{H}, \mathrm{H}_{6}, \mathrm{H}_{7}\right) ; 3.12\left(\mathrm{~d}, J=12.53 \mathrm{~Hz}, 2 \mathrm{H}, \mathrm{H}_{5}\right) .{ }^{13} \mathrm{C} \mathrm{NMR}\left(100 \mathrm{MHz}, \mathrm{CD}_{3} \mathrm{CN}\right) \delta[\mathrm{ppm}]: 76.59(\mathrm{~d}$, $\left.J=2.54 \mathrm{~Hz}, 1 \mathrm{C}, \mathrm{C}_{4}\right) ; 74.19\left(\mathrm{~d}, J=1.77 \mathrm{~Hz}, 2 \mathrm{C}, \mathrm{C}_{3}\right) ; 73.46\left(2 \mathrm{C}, \mathrm{C}_{2}\right) ; 72.35\left(\mathrm{~d}, J=9.44 \mathrm{~Hz}, 3 \mathrm{C}, \mathrm{C}_{7}\right) ; 62.63(6 \mathrm{C}$, $\left.\mathrm{C}_{1}\right) ; 47.71$ (d, $\left.J=29.75 \mathrm{~Hz}, 3 \mathrm{C}, \mathrm{C}_{6}\right) ; 24.25$ (d, $\left.J=16.38 \mathrm{~Hz}, 1 \mathrm{C}, \mathrm{C}_{5}\right) .{ }^{31} \mathrm{P} \mathrm{NMR}\left(162 \mathrm{MHz}, \mathrm{CD}_{3} \mathrm{CN}\right) \delta[\mathrm{ppm}]:$

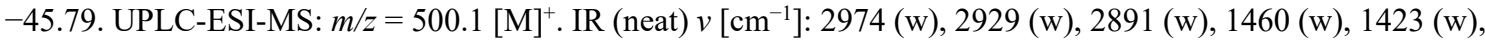
1258 (s), 1241 (s), 1225 (s), 1157 (s), 1097 (w), 1028 (s), 1013 (m), 968 (s), 943 (m), 916 (m), 880 (w), 852 (w), 816 (m), 786 (m). HR-ESI-MS $m / z$ : [M] ${ }^{+}$calcd for $\mathrm{C}_{18} \mathrm{H}_{24} \mathrm{~N}_{3} \mathrm{PRe}, 500.12599$; found, 500.12582. 


\section{Spectra}

Signal assignments in the following NMR spectra are given according to numbering depicted in the respective spectra. Additional assignable peaks, that do not belong to the spectrum of the desired compound, are labeled with special characters as following:

$\diamond \quad$ residual solvent peak(s)

a $\quad \mathrm{H}_{2} \mathrm{O}$ signal

$+\quad$ HDO signal

$\Delta \quad \mathrm{H} /$ silicone grease signal(s)

\section{$3.1\left[\operatorname{Re}\left(\eta^{5}-\mathrm{C}_{5} \mathrm{H}_{4} \mathrm{CH}_{2} \mathrm{OH}\right)\left(\eta^{6}-\mathrm{C}_{6} \mathrm{H}_{6}\right)\right](2)$}

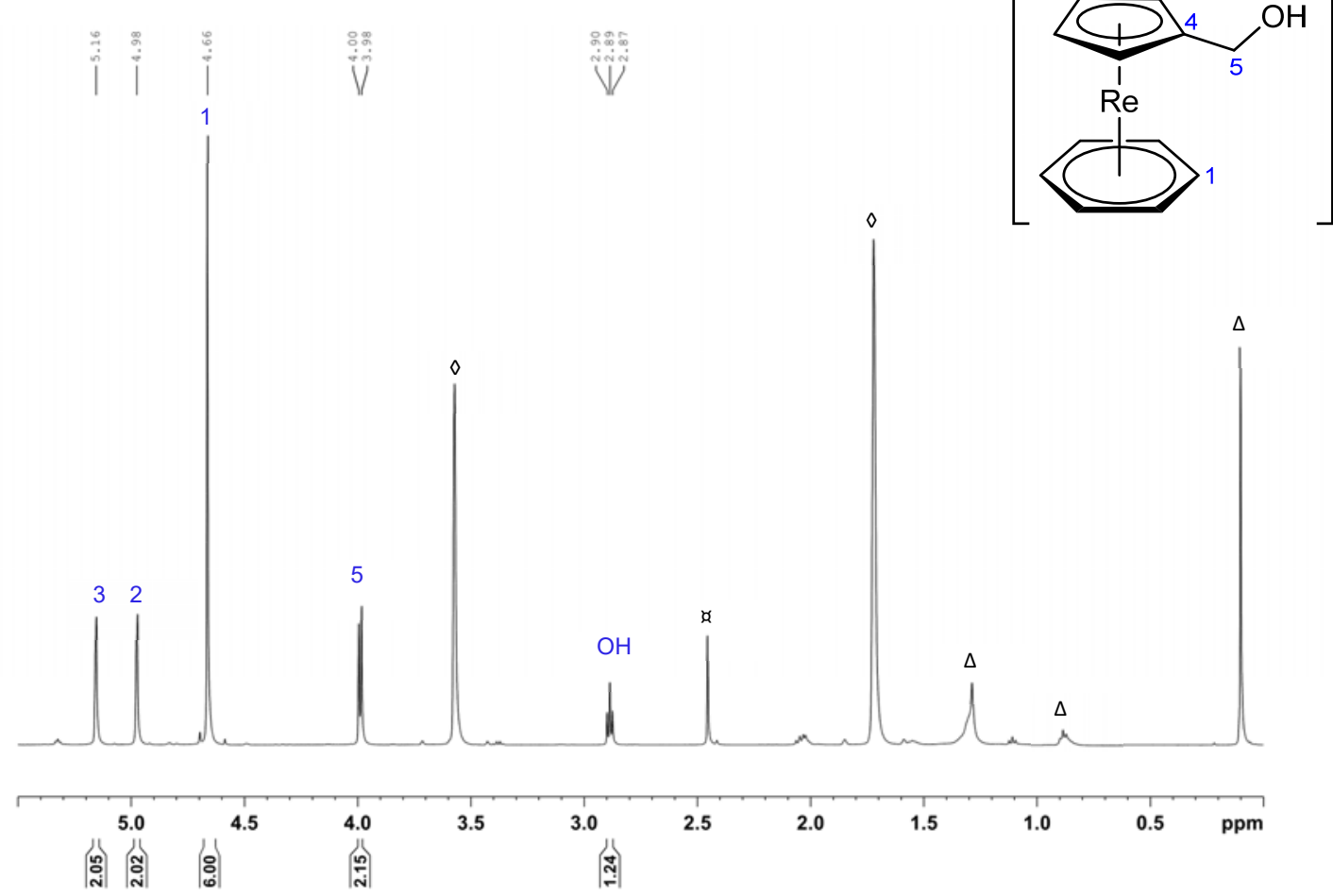

Figure S1. ${ }^{1} \mathrm{H}$ NMR spectrum of $\left[\mathrm{Re}\left(\eta^{5}-\mathrm{C}_{5} \mathrm{H}_{4} \mathrm{CH}_{2} \mathrm{OH}\right)\left(\eta^{6}-\mathrm{C}_{6} \mathrm{H}_{6}\right)\right](2)\left(500 \mathrm{MHz}, \mathrm{C}_{4} \mathrm{D}_{8} \mathrm{O}\right)$. 


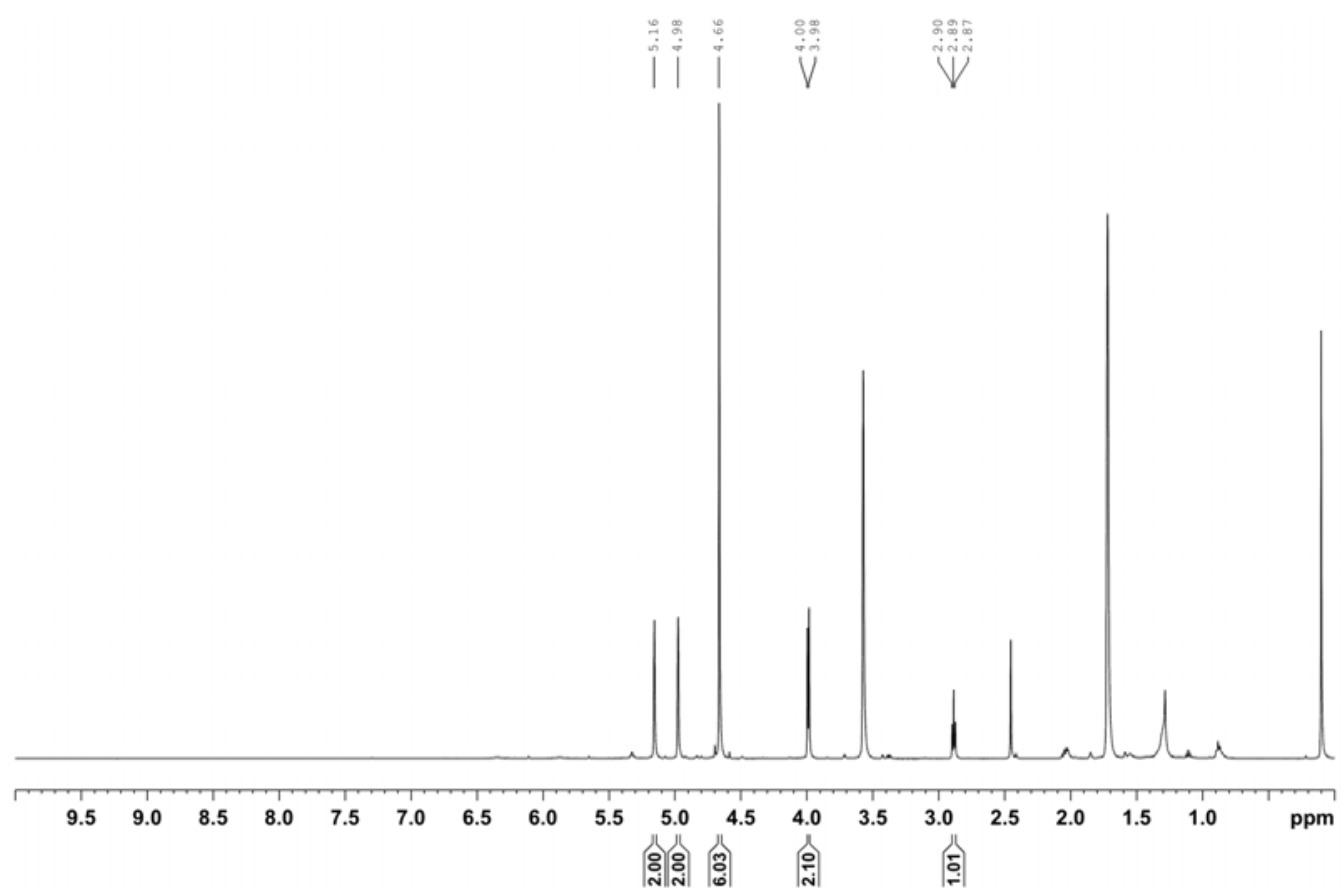

Figure S2. Full ${ }^{1} \mathrm{H}$ NMR spectrum of $\left[\mathrm{Re}\left(\eta^{5}-\mathrm{C}_{5} \mathrm{H}_{4} \mathrm{CH}_{2} \mathrm{OH}\right)\left(\eta^{6}-\mathrm{C}_{6} \mathrm{H}_{6}\right)\right](2)\left(500 \mathrm{MHz}, \mathrm{C}_{4} \mathrm{D}_{8} \mathrm{O}\right)$.

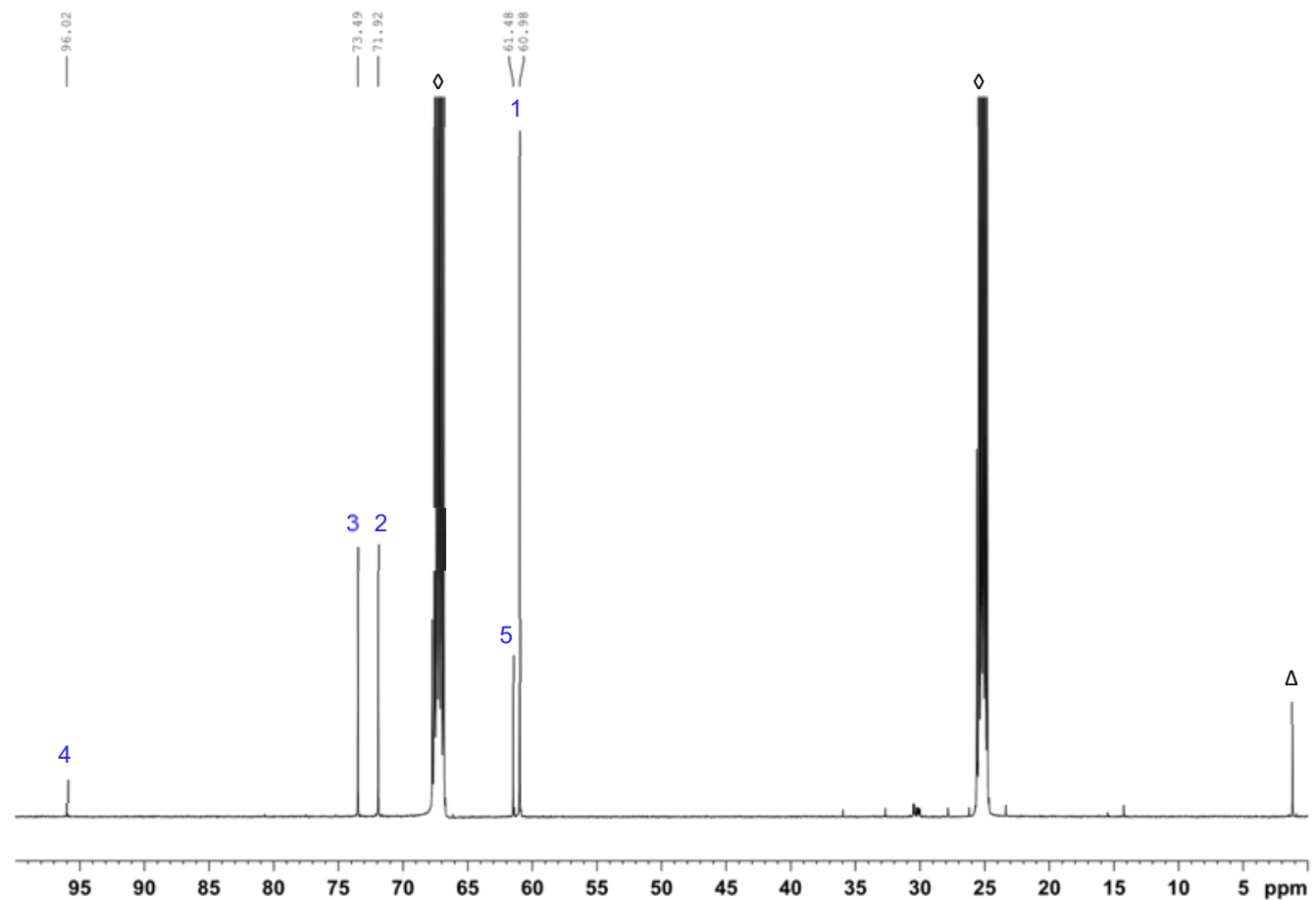

Figure S3. ${ }^{13} \mathrm{C}$ NMR spectrum of $\left[\mathrm{Re}\left(\eta^{5}-\mathrm{C}_{5} \mathrm{H}_{4} \mathrm{CH}_{2} \mathrm{OH}\right)\left(\eta^{6}-\mathrm{C}_{6} \mathrm{H}_{6}\right)\right](2)\left(125 \mathrm{MHz}, \mathrm{C}_{4} \mathrm{D}_{8} \mathrm{O}\right)$. 


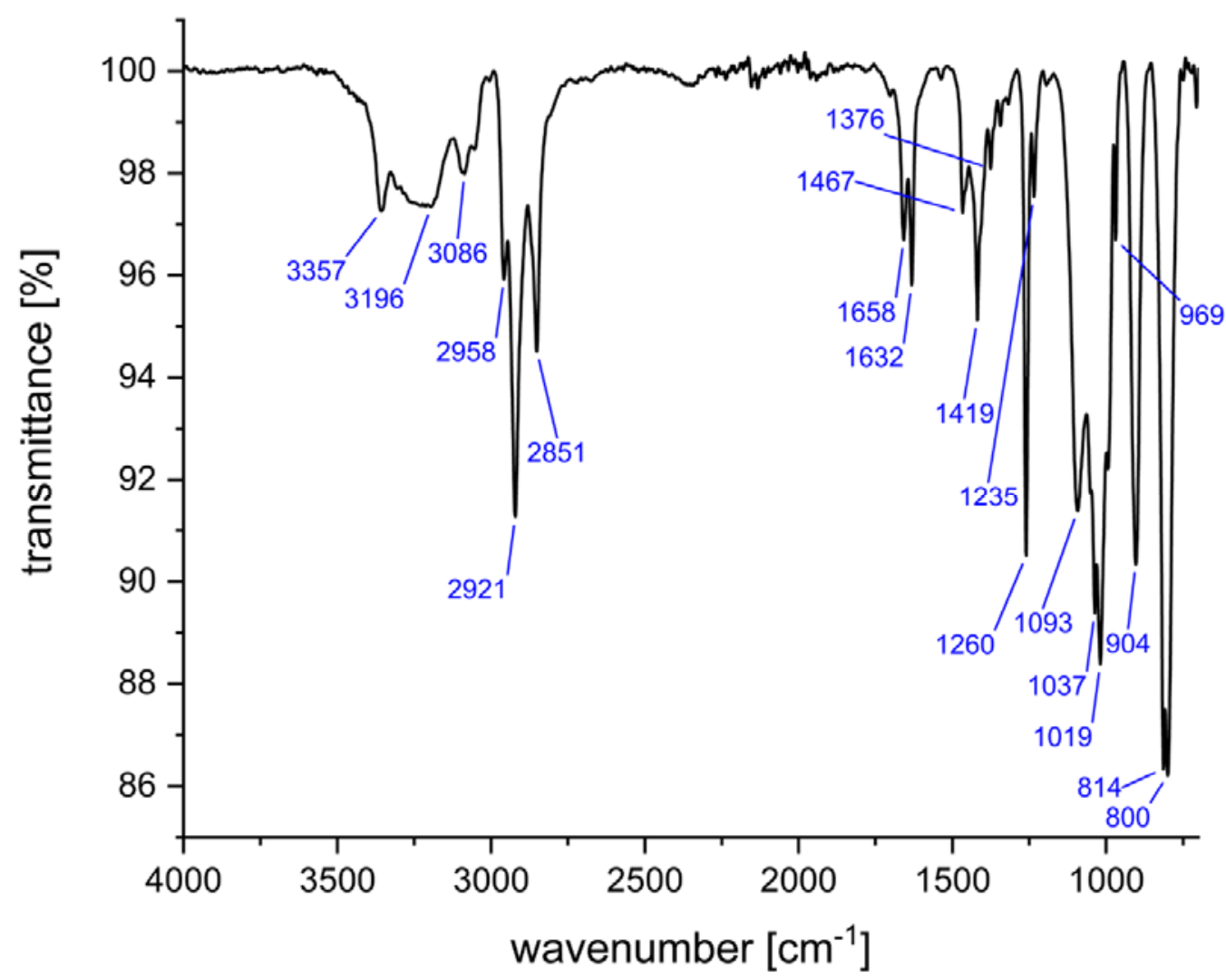

Figure S4. IR spectrum of $\left[\mathrm{Re}\left(\eta^{5}-\mathrm{C}_{5} \mathrm{H}_{4} \mathrm{CH}_{2} \mathrm{OH}\right)\left(\eta^{6}-\mathrm{C}_{6} \mathrm{H}_{6}\right)\right]$ (2) (neat).

\section{$3.2\left[\operatorname{Re}\left(\eta^{6}-\mathrm{C}_{5} \mathrm{H}_{4} \mathrm{CH}_{2}\right)\left(\eta^{6}-\mathrm{C}_{6} \mathrm{H}_{6}\right)\right](\mathrm{OTf})([3](\mathrm{OTf}))$}

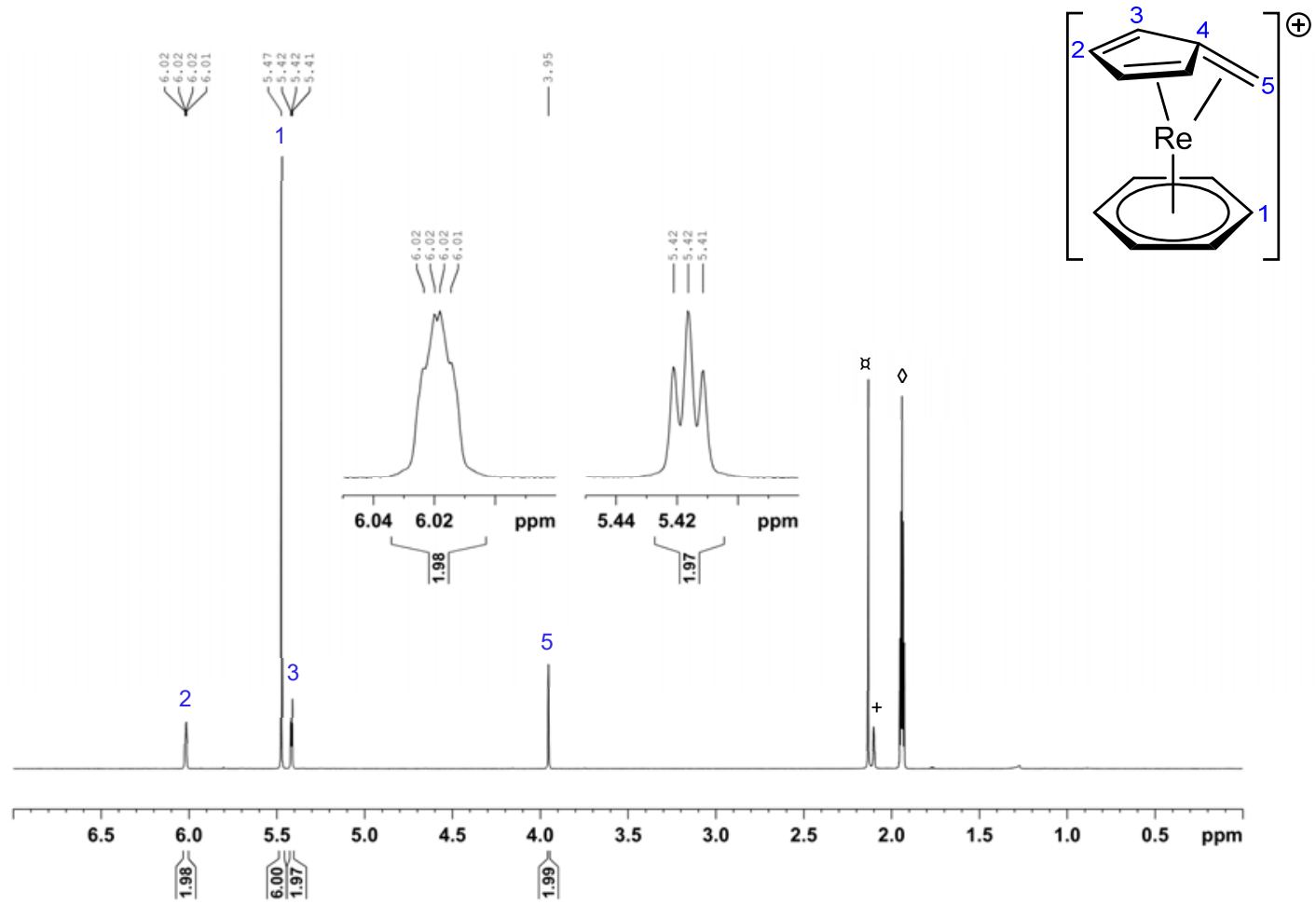

Figure S5. ${ }^{1} \mathrm{H}$ NMR spectrum of $\left[\mathrm{Re}\left(\eta^{6}-\mathrm{C}_{5} \mathrm{H}_{4} \mathrm{CH}_{2}\right)\left(\eta^{6}-\mathrm{C}_{6} \mathrm{H}_{6}\right)\right](\mathrm{OTf})([3](\mathrm{OTf}))\left(400 \mathrm{MHz}, \mathrm{CD}_{3} \mathrm{CN}\right)$. 


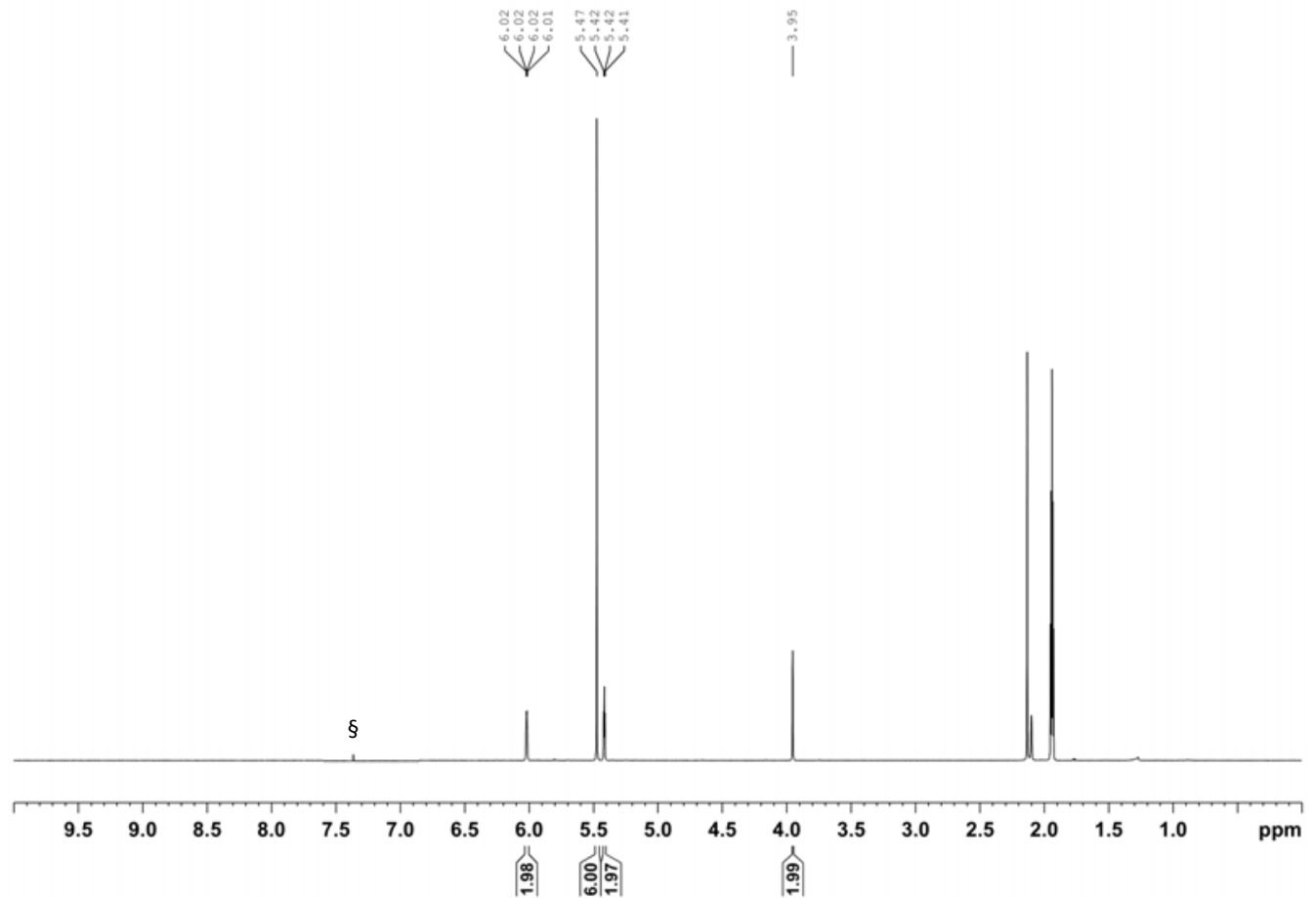

Figure S6. Full ${ }^{1} \mathrm{H}$ NMR spectrum of $\left[\mathrm{Re}\left(\eta^{6}-\mathrm{C}_{5} \mathrm{H}_{4} \mathrm{CH}_{2}\right)\left(\eta^{6}-\mathrm{C}_{6} \mathrm{H}_{6}\right)\right](\mathrm{OTf})([3](\mathrm{OTf}))\left(400 \mathrm{MHz}, \mathrm{CD}_{3} \mathrm{CN}\right)$. (§: benzene).

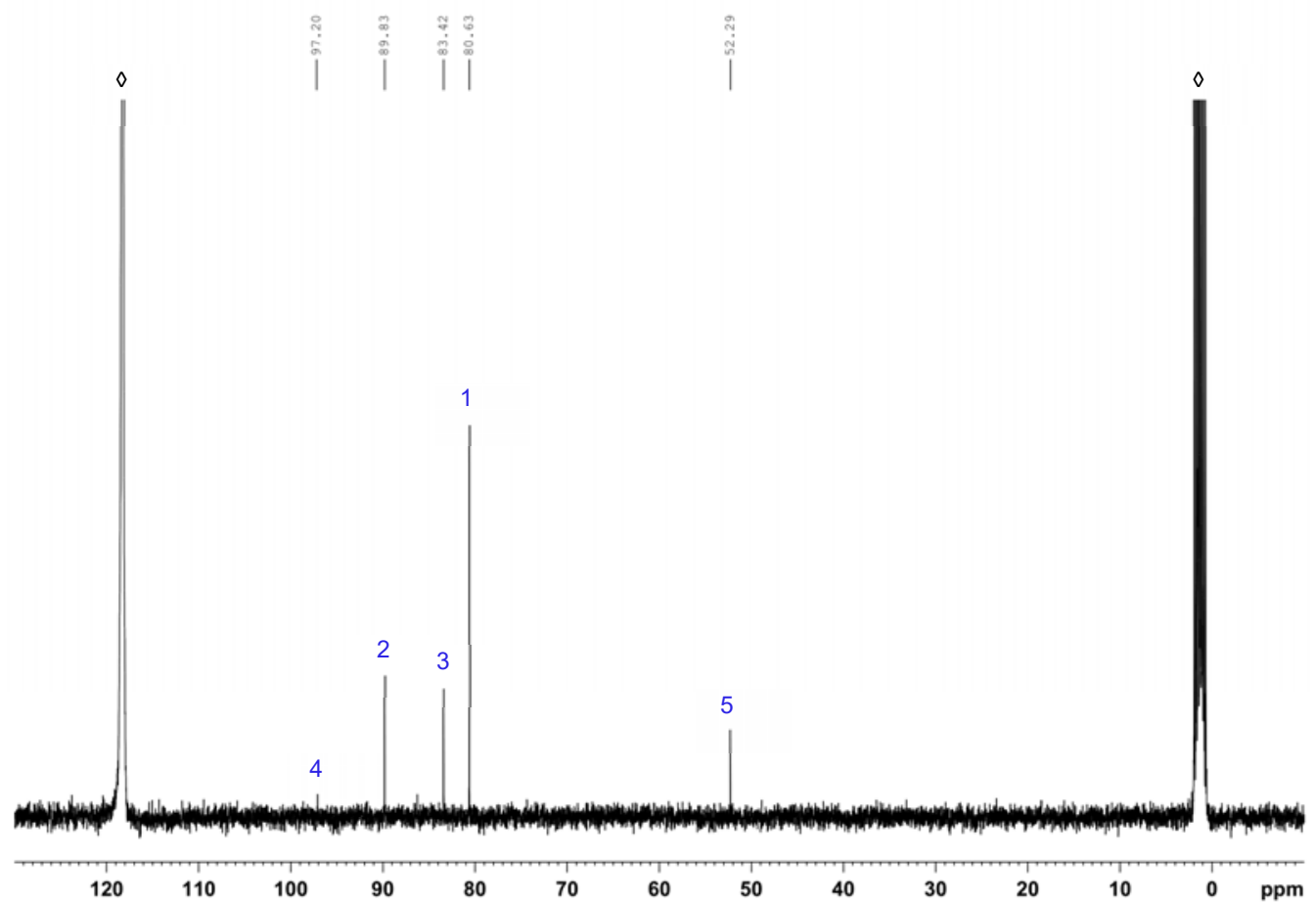

Figure S7. ${ }^{13} \mathrm{C}$ NMR spectrum of $\left[\mathrm{Re}\left(\eta^{6}-\mathrm{C}_{5} \mathrm{H}_{4} \mathrm{CH}_{2}\right)\left(\eta^{6}-\mathrm{C}_{6} \mathrm{H}_{6}\right)\right](\mathrm{OTf})([3](\mathrm{OTf}))\left(100 \mathrm{MHz}, \mathrm{CD}_{3} \mathrm{CN}\right)$. 


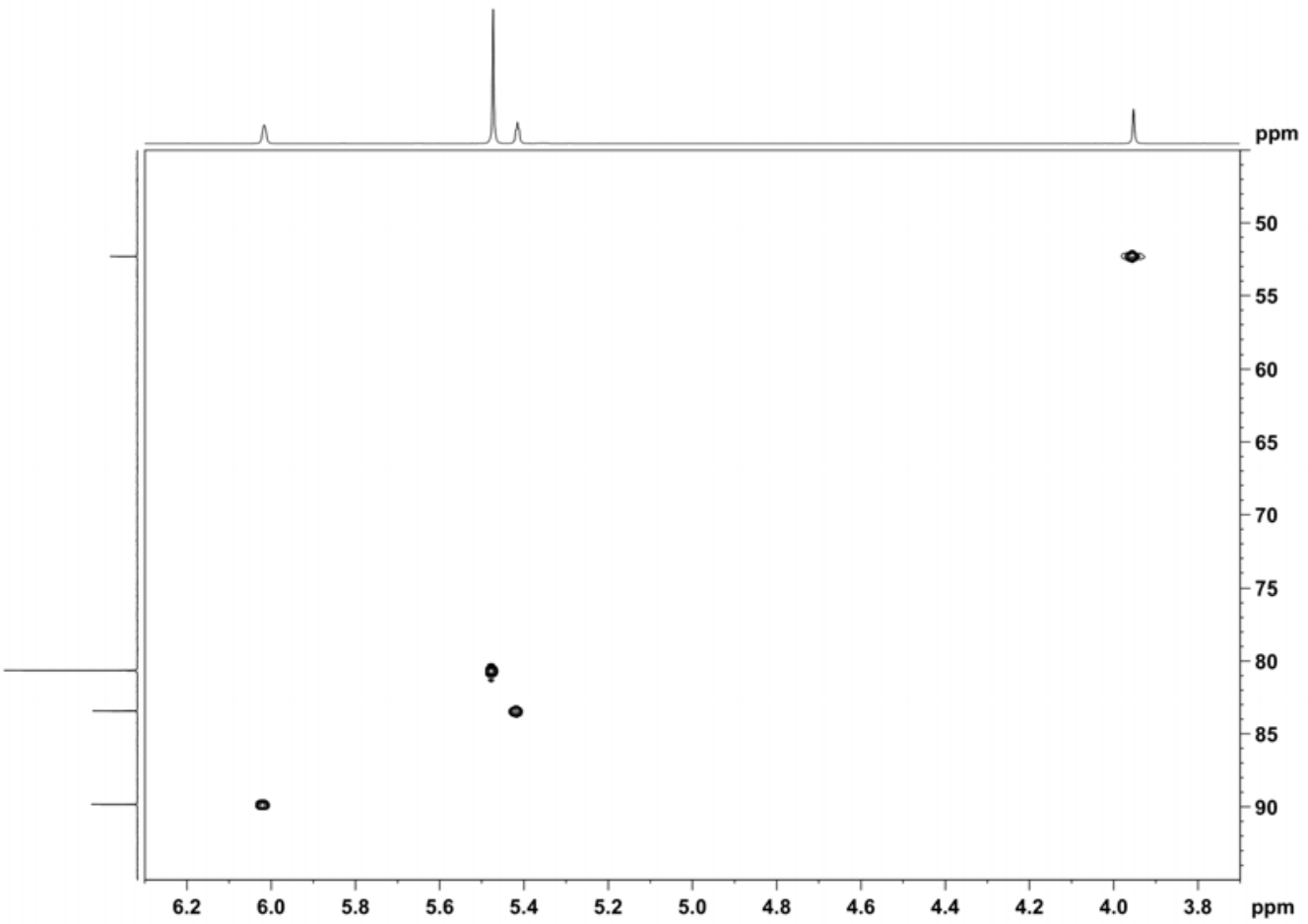

Figure S8. Relevant region in the HSQC spectrum of $\left[\mathrm{Re}\left(\eta^{6}-\mathrm{C}_{5} \mathrm{H}_{4} \mathrm{CH}_{2}\right)\left(\eta^{6}-\mathrm{C}_{6} \mathrm{H}_{6}\right)\right](\mathrm{OTf}) \quad([3](\mathrm{OTf}))$ $\left(500 / 125 \mathrm{MHz}, \mathrm{CD}_{3} \mathrm{CN}\right)$.

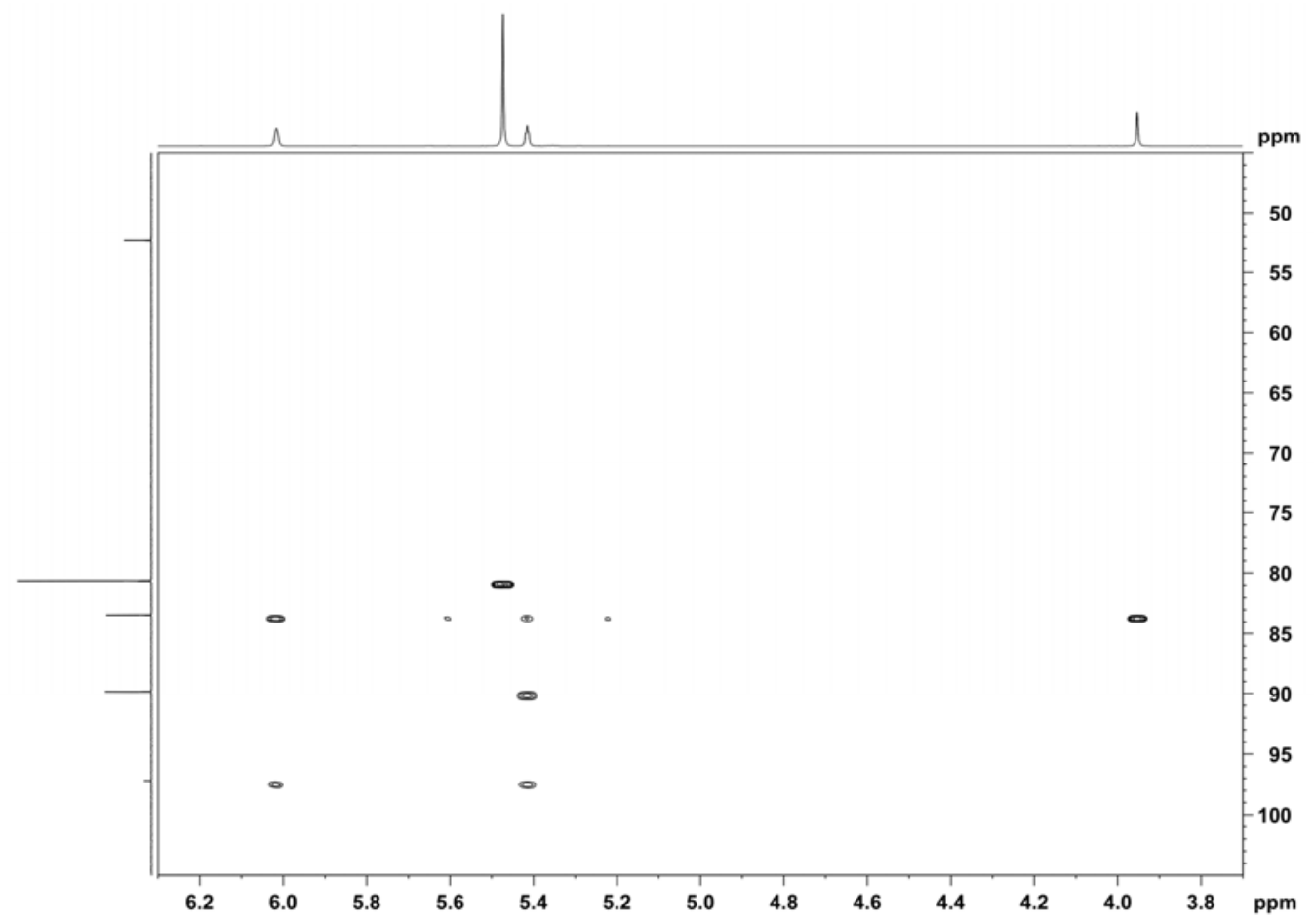

Figure S9. Relevant region in the $\mathrm{HMBC}$ spectrum of $\left[\mathrm{Re}\left(\eta^{6}-\mathrm{C}_{5} \mathrm{H}_{4} \mathrm{CH}_{2}\right)\left(\eta^{6}-\mathrm{C}_{6} \mathrm{H}_{6}\right)\right](\mathrm{OTf})$ ([3](OTf)) $\left(500 / 125 \mathrm{MHz}, \mathrm{CD}_{3} \mathrm{CN}\right)$. 


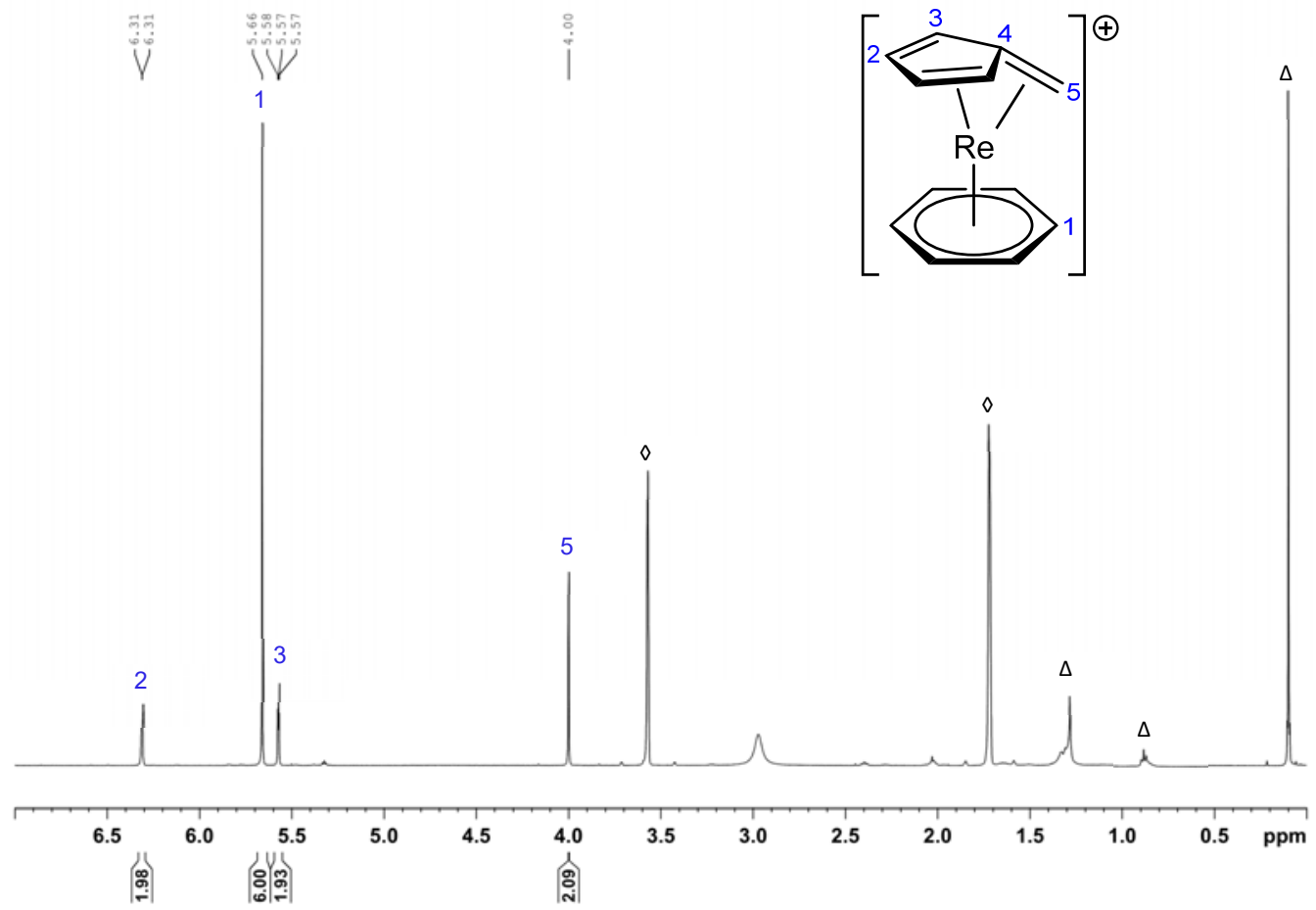

Figure S10. ${ }^{1} \mathrm{H}$ NMR spectrum of $\left[\mathrm{Re}\left(\eta^{6}-\mathrm{C}_{5} \mathrm{H}_{4} \mathrm{CH}_{2}\right)\left(\eta^{6}-\mathrm{C}_{6} \mathrm{H}_{6}\right)\right](\mathrm{OTf})([3](\mathrm{OTf}))\left(500 \mathrm{MHz}, \mathrm{C}_{4} \mathrm{D}_{8} \mathrm{O}\right)$.

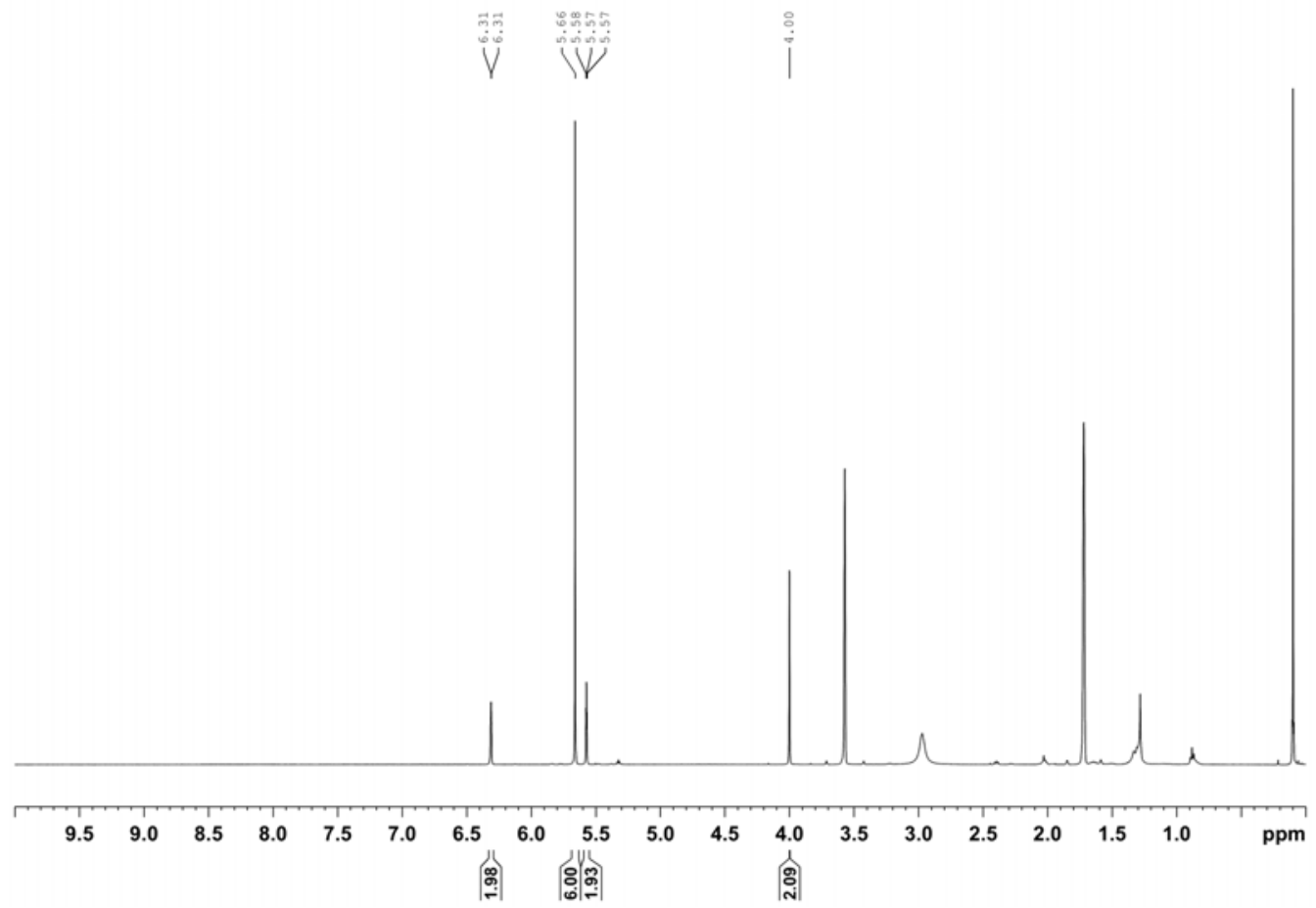

Figure S11. Full ${ }^{1} \mathrm{H}$ NMR spectrum of $\left[\mathrm{Re}\left(\eta^{6}-\mathrm{C}_{5} \mathrm{H}_{4} \mathrm{CH}_{2}\right)\left(\eta^{6}-\mathrm{C}_{6} \mathrm{H}_{6}\right)\right](\mathrm{OTf})([3](\mathrm{OTf}))\left(500 \mathrm{MHz}, \mathrm{C}_{4} \mathrm{D}_{8} \mathrm{O}\right)$. 


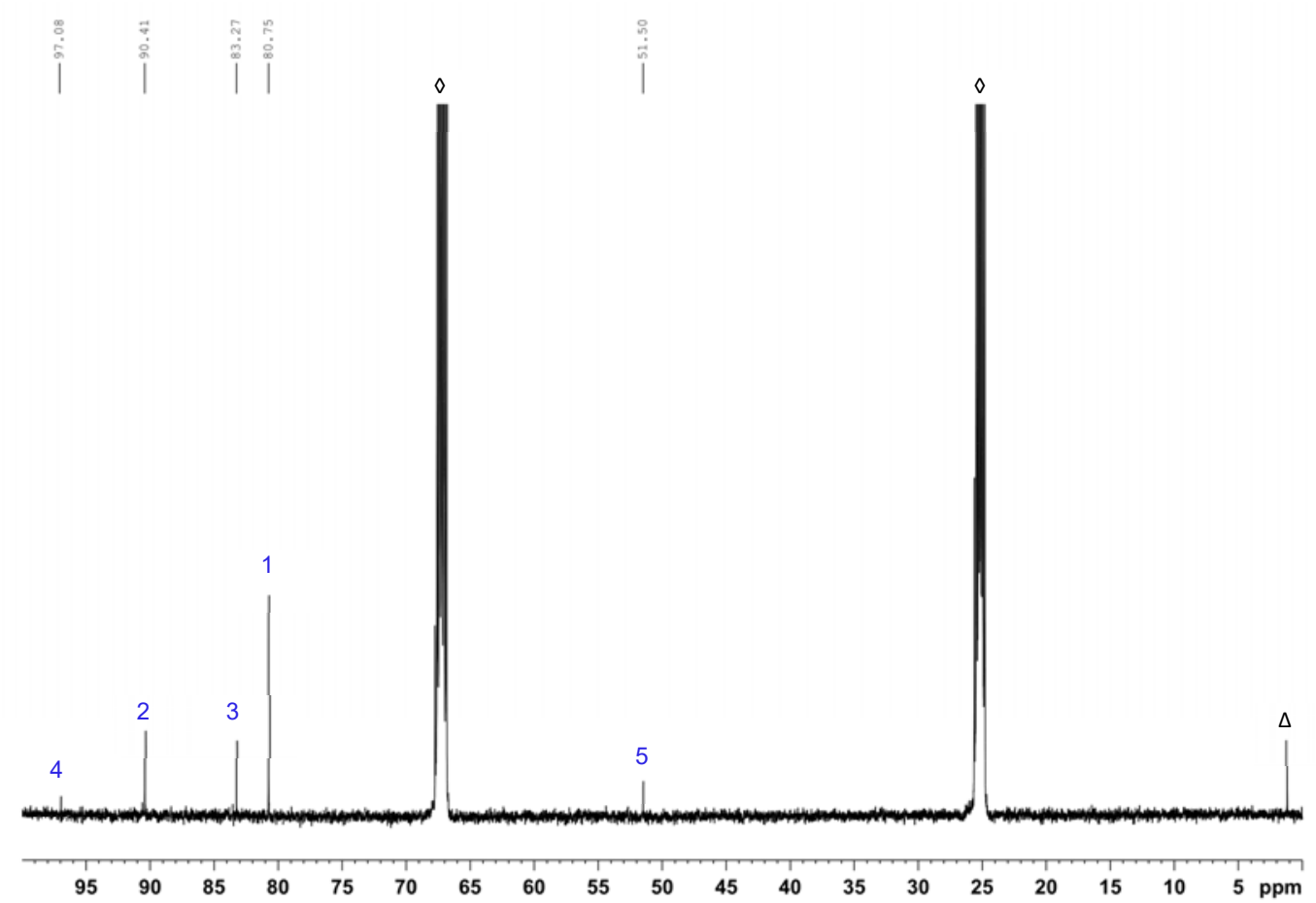

Figure S12. ${ }^{13} \mathrm{C}$ NMR spectrum of $\left[\mathrm{Re}\left(\eta^{6}-\mathrm{C}_{5} \mathrm{H}_{4} \mathrm{CH}_{2}\right)\left(\eta^{6}-\mathrm{C}_{6} \mathrm{H}_{6}\right)\right](\mathrm{OTf})([3](\mathrm{OTf}))\left(125 \mathrm{MHz}, \mathrm{C}_{4} \mathrm{D}_{8} \mathrm{O}\right)$.

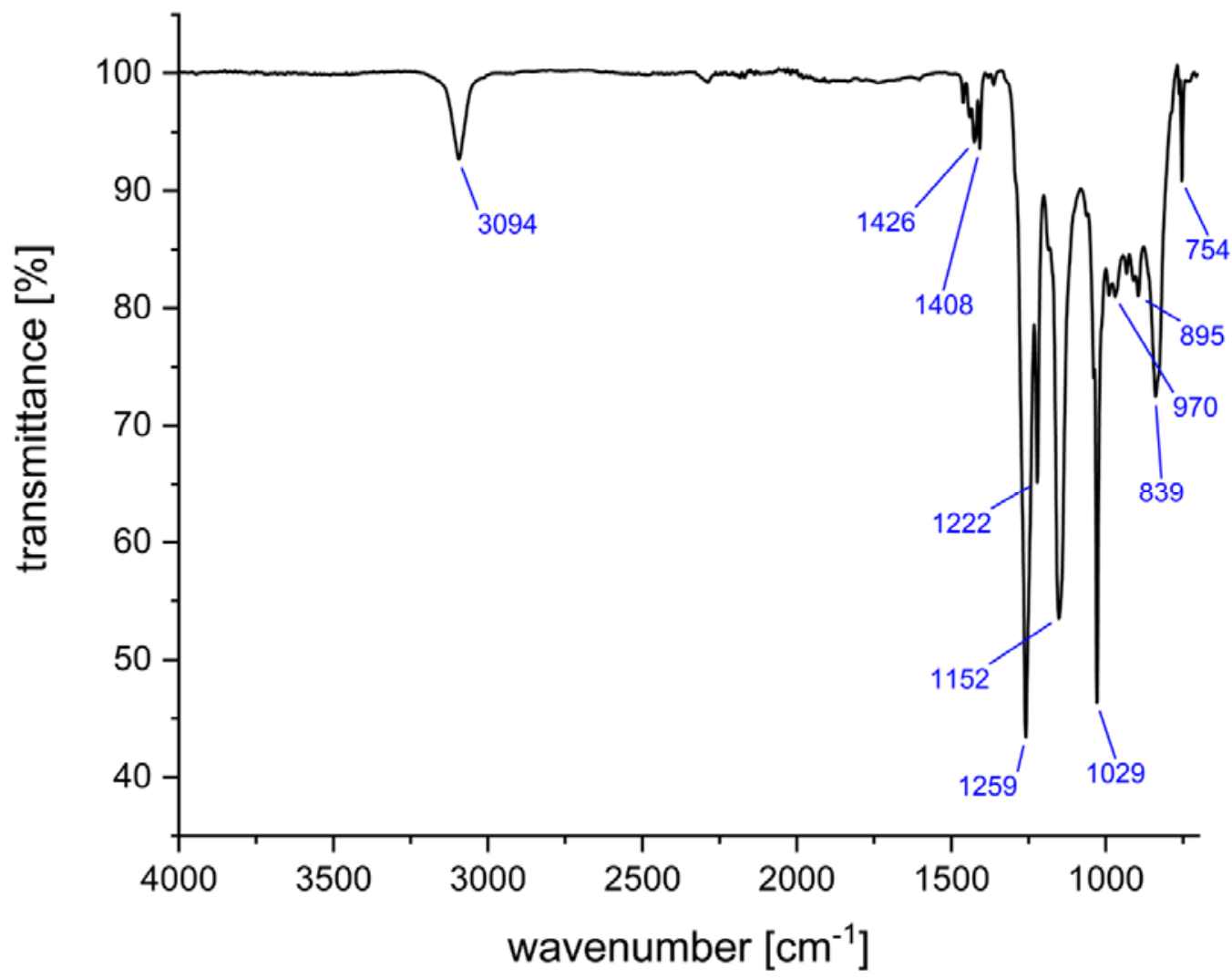

Figure S13. IR spectrum of $\left[\mathrm{Re}\left(\eta^{6}-\mathrm{C}_{5} \mathrm{H}_{4} \mathrm{CH}_{2}\right)\left(\eta^{6}-\mathrm{C}_{6} \mathrm{H}_{6}\right)\right](\mathrm{OTf})([3](\mathrm{OTf}))$ (neat). 


\section{$3.3 \quad\left[\operatorname{Re}\left(\eta^{5}-\mathrm{C}_{5} \mathrm{H}_{4} \mathrm{CH}=\mathrm{NCH}_{2} \mathrm{C}_{6} \mathrm{H}_{5}\right)\left(\eta^{6}-\mathrm{C}_{6} \mathrm{H}_{6}\right)\right](4)$}

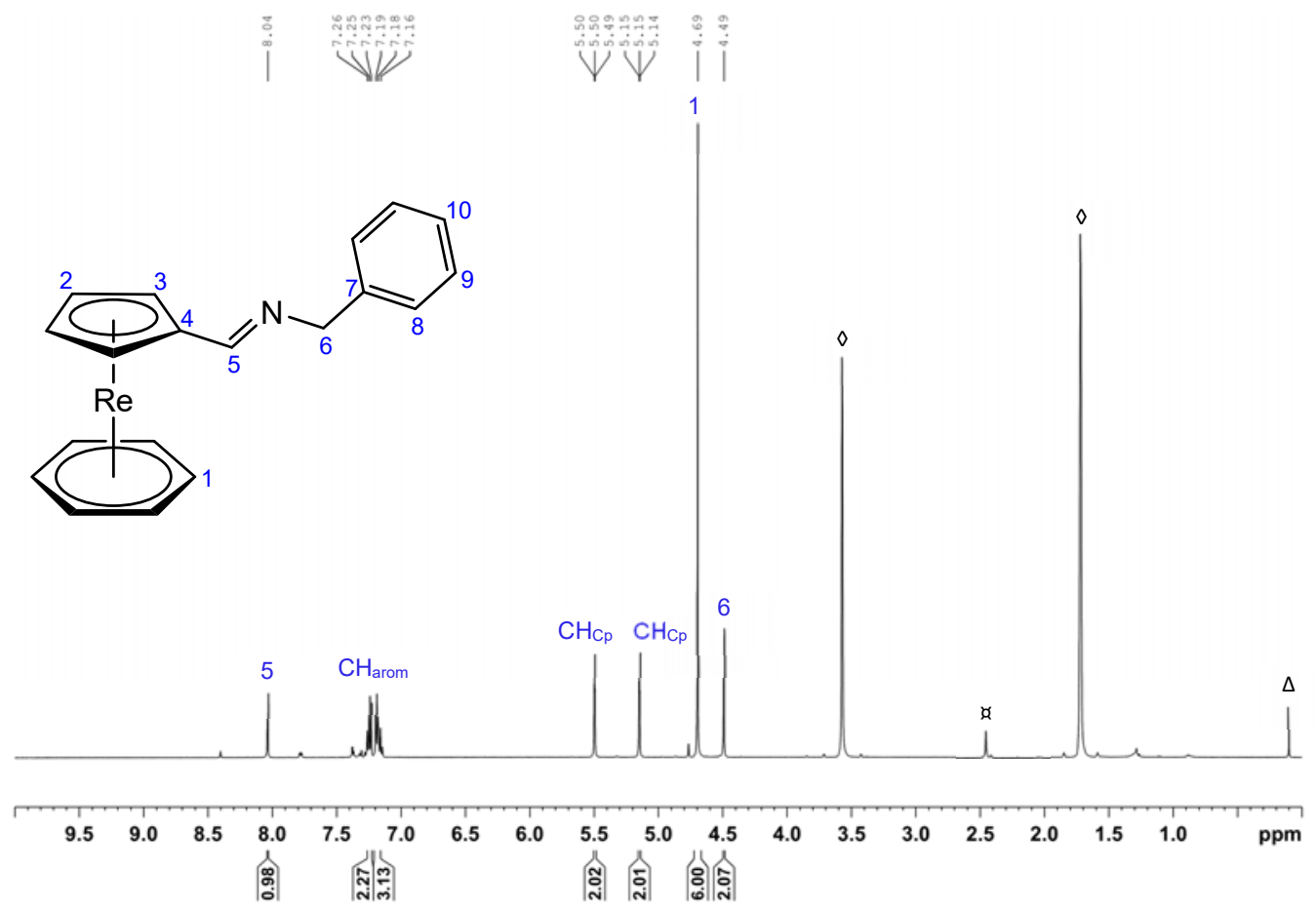

Figure S14. Full ${ }^{1} \mathrm{H}$ NMR spectrum of $\left[\mathrm{Re}\left(\eta^{6}-\mathrm{C}_{5} \mathrm{H}_{4} \mathrm{CH}=\mathrm{NCH}_{2} \mathrm{C}_{6} \mathrm{H}_{5}\right)\left(\eta^{6}-\mathrm{C}_{6} \mathrm{H}_{6}\right)\right](4)\left(500 \mathrm{MHz}, \mathrm{C}_{4} \mathrm{D}_{8} \mathrm{O}\right)$.

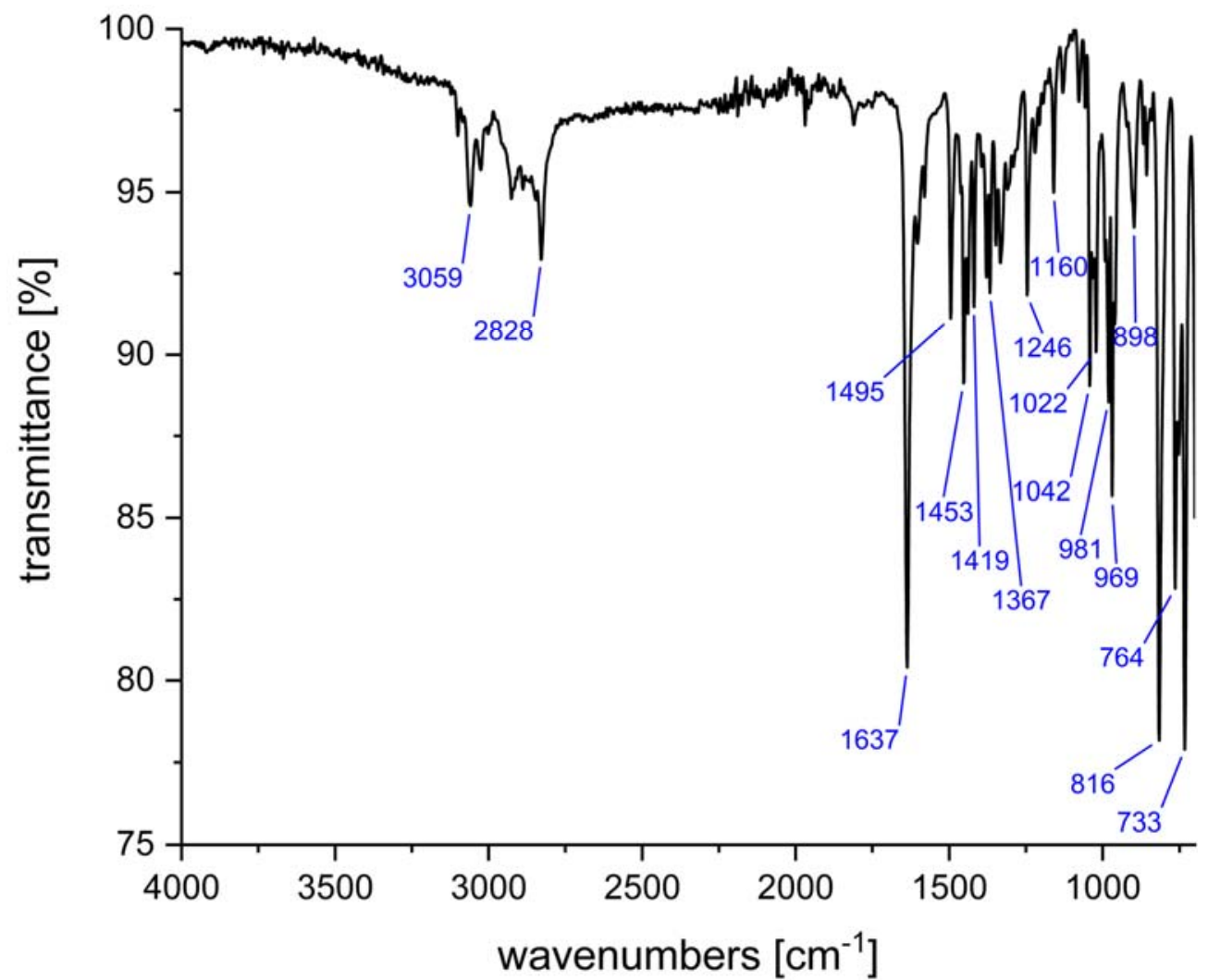

Figure S15. IR spectrum of $\left[\mathrm{Re}\left(\eta^{5}-\mathrm{C}_{5} \mathrm{H}_{4} \mathrm{CH}=\mathrm{NCH}_{2} \mathrm{C}_{6} \mathrm{H}_{5}\right)\left(\eta^{6}-\mathrm{C}_{6} \mathrm{H}_{6}\right)\right]$ (4) (neat). 


\section{$3.4\left[\operatorname{Re}\left(\eta^{5}-\mathrm{C}_{5} \mathrm{H}_{4} \mathrm{CH}_{2} \mathrm{H}\right)\left(\eta^{6}-\mathrm{C}_{6} \mathrm{H}_{6}\right)\right](5)$}

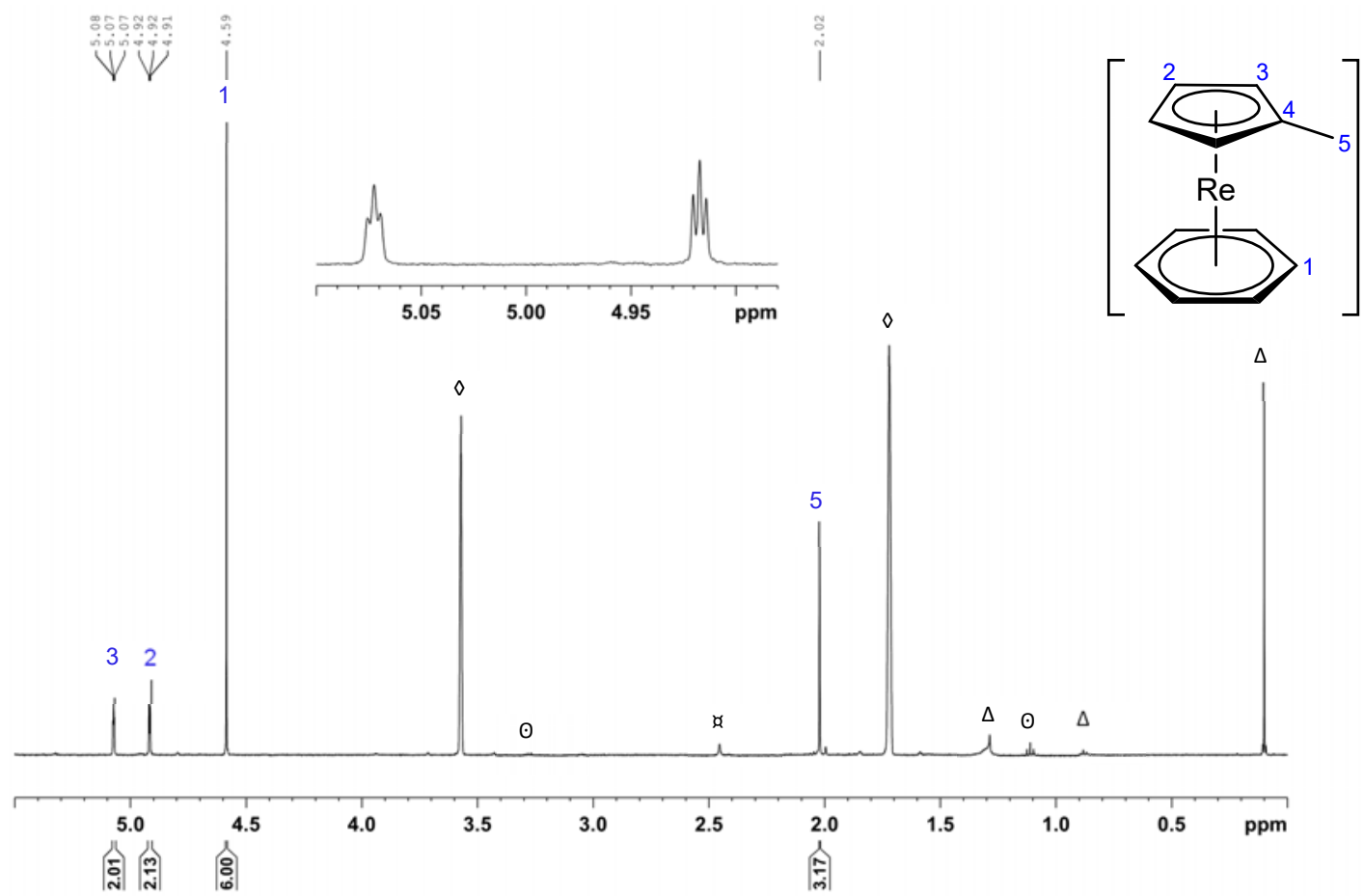

Figure S16. ${ }^{1} \mathrm{H}$ NMR spectrum of $\left[\mathrm{Re}\left(\eta^{5}-\mathrm{C}_{5} \mathrm{H}_{4} \mathrm{CH}_{2} \mathrm{H}\right)\left(\eta^{6}-\mathrm{C}_{6} \mathrm{H}_{6}\right)\right]\left(\right.$ 5) $\left(500 \mathrm{MHz}, \mathrm{C}_{4} \mathrm{D}_{8} \mathrm{O}\right)$. ( $\left(\odot: \mathrm{Et}_{2} \mathrm{O}\right.$ signals).

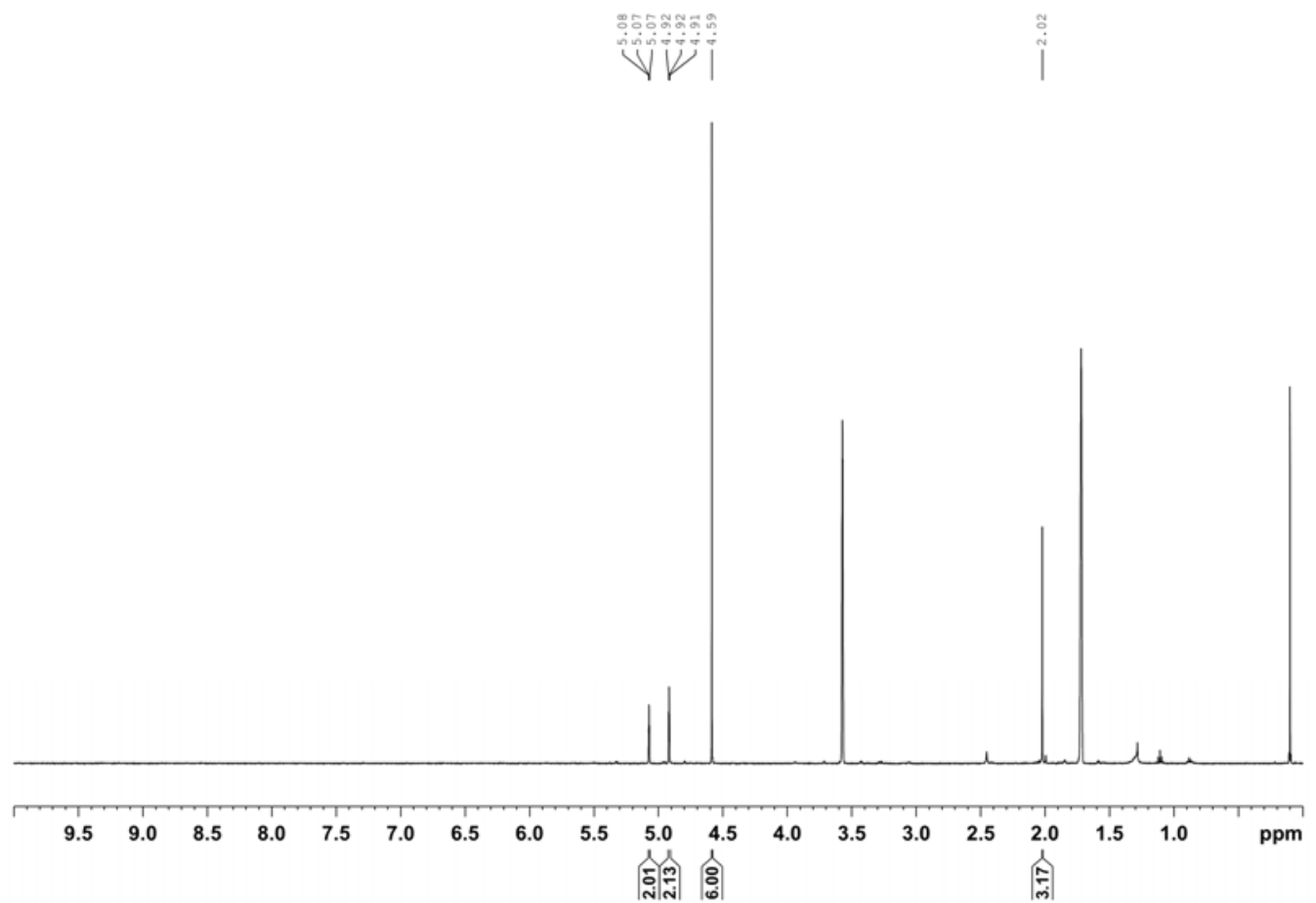

Figure S17. Full ${ }^{1} \mathrm{H}$ NMR spectrum of $\left[\mathrm{Re}\left(\eta^{5}-\mathrm{C}_{5} \mathrm{H}_{4} \mathrm{CH}_{2} \mathrm{H}\right)\left(\eta^{6}-\mathrm{C}_{6} \mathrm{H}_{6}\right)\right]$ (5) (500 MHz, $\left.\mathrm{C}_{4} \mathrm{D}_{8} \mathrm{O}\right)$. ( $\left(\odot: \mathrm{Et}_{2} \mathrm{O}\right.$ signals). 


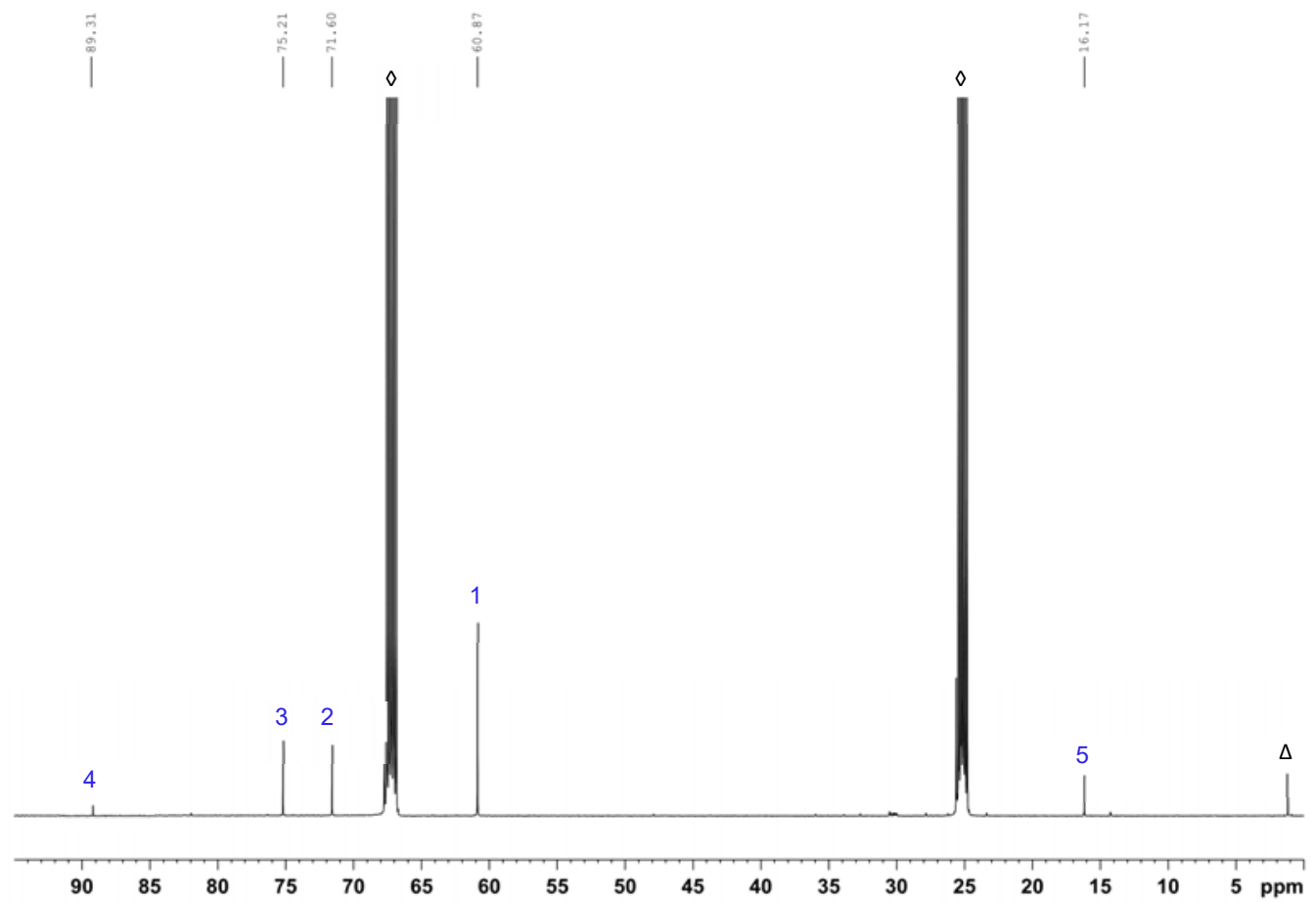

Figure S18. ${ }^{13} \mathrm{C}$ NMR spectrum of $\left[\mathrm{Re}\left(\eta^{5}-\mathrm{C}_{5} \mathrm{H}_{4} \mathrm{CH}_{2} \mathrm{H}\right)\left(\eta^{6}-\mathrm{C}_{6} \mathrm{H}_{6}\right)\right]\left(\right.$ 5) $\left(125 \mathrm{MHz}, \mathrm{C}_{4} \mathrm{D}_{8} \mathrm{O}\right)$.

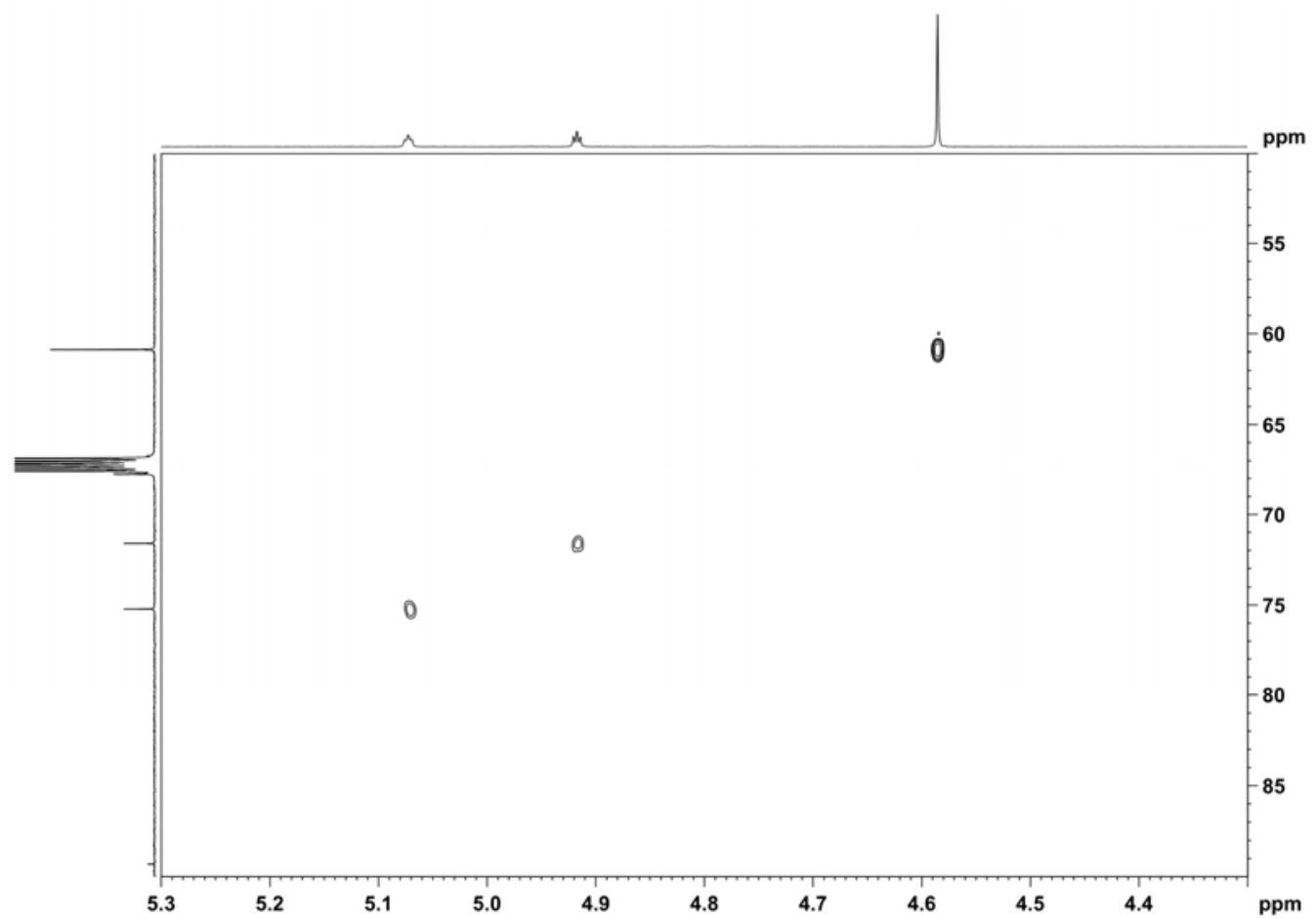

Figure S19. Relevant region in the HSQC spectrum of $\left[R e\left(\eta^{5}-\mathrm{C}_{5} \mathrm{H}_{4} \mathrm{CH}_{2} \mathrm{H}\right)\left(\eta^{6}-\mathrm{C}_{6} \mathrm{H}_{6}\right)\right](5)\left(500 / 125 \mathrm{MHz}, \mathrm{C}_{4} \mathrm{D}_{8} \mathrm{O}\right)$. 


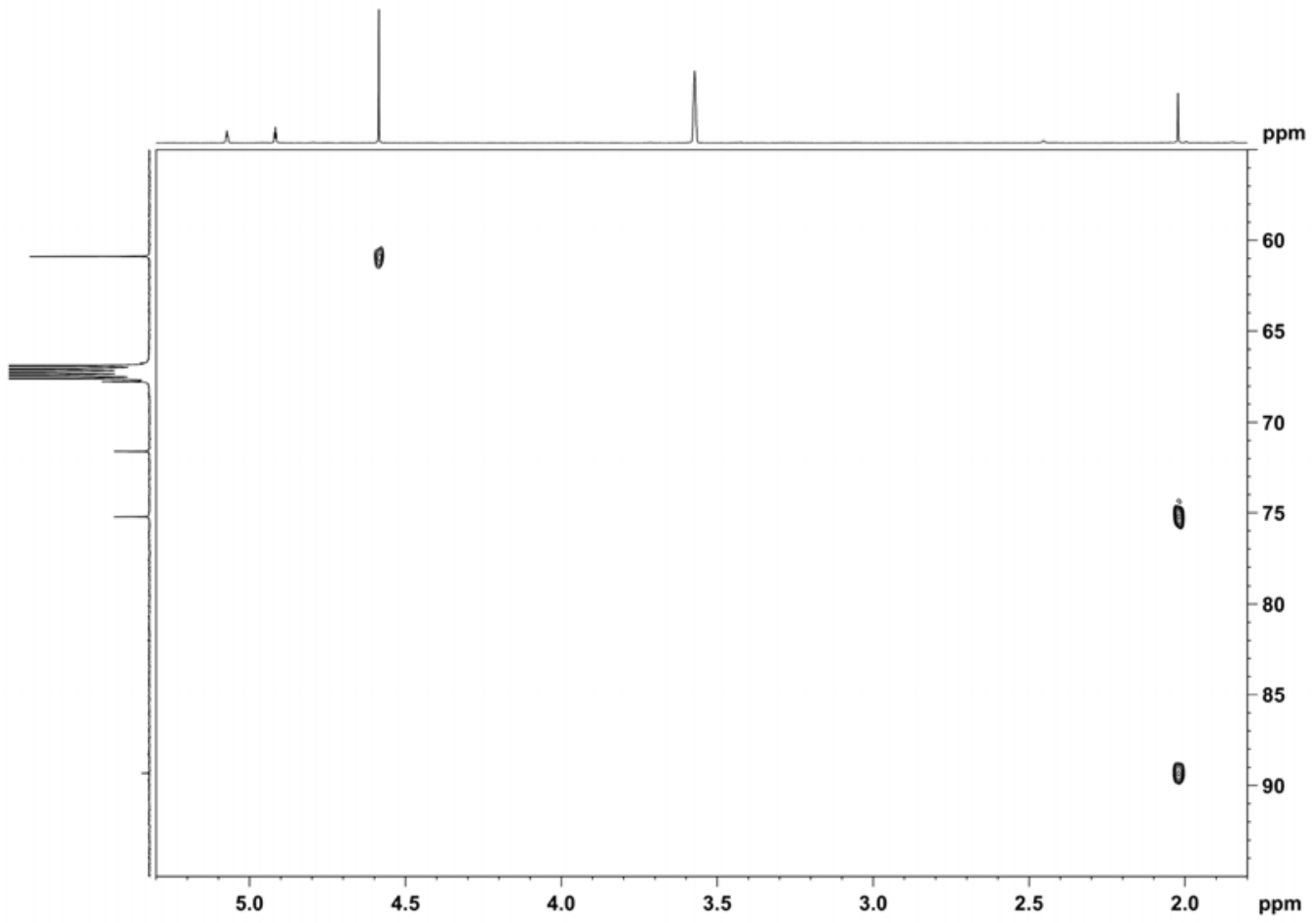

Figure S20. Relevant region in the HMBC spectrum of $\left[\mathrm{Re}\left(\eta^{5}-\mathrm{C}_{5} \mathrm{H}_{4} \mathrm{CH}_{2} \mathrm{H}\right)\left(\eta^{6}-\mathrm{C}_{6} \mathrm{H}_{6}\right)\right](5)\left(500 \mathrm{MHz}, \mathrm{C}_{4} \mathrm{D}_{8} \mathrm{O}\right)$.

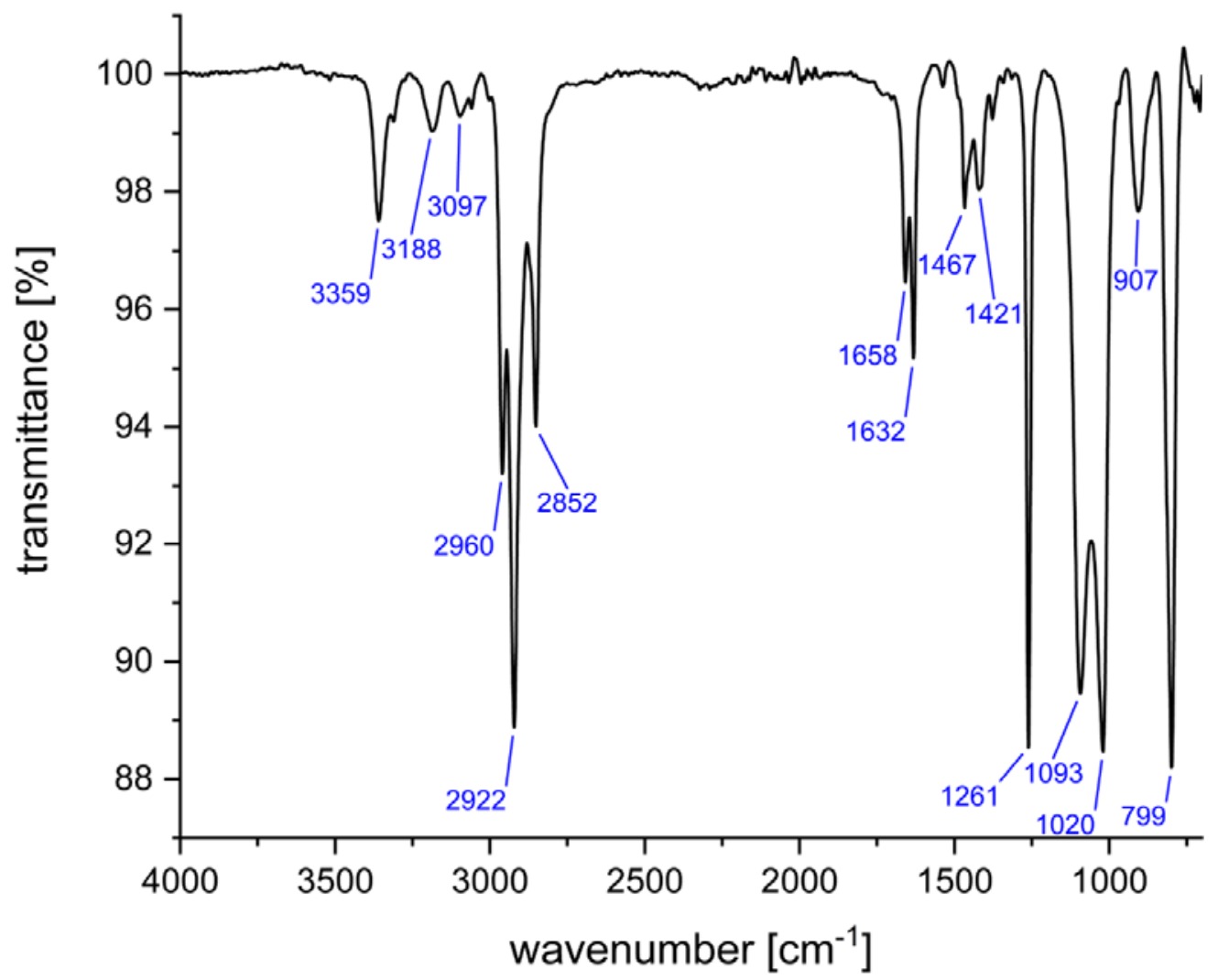

Figure S21. IR spectrum of $\left[\mathrm{Re}\left(\eta^{5}-\mathrm{C}_{5} \mathrm{H}_{4} \mathrm{CH}_{2} \mathrm{H}\right)\left(\eta^{6}-\mathrm{C}_{6} \mathrm{H}_{6}\right)\right]$ (5) (neat). 


\section{$3.5 \quad\left[\operatorname{Re}\left(\boldsymbol{\eta}^{5}-\mathrm{C}_{5} \mathrm{H}_{4} \mathrm{CH}_{2} \mathrm{CN}\right)\left(\boldsymbol{\eta}^{6}-\mathrm{C}_{6} \mathrm{H}_{6}\right)\right](6)$}

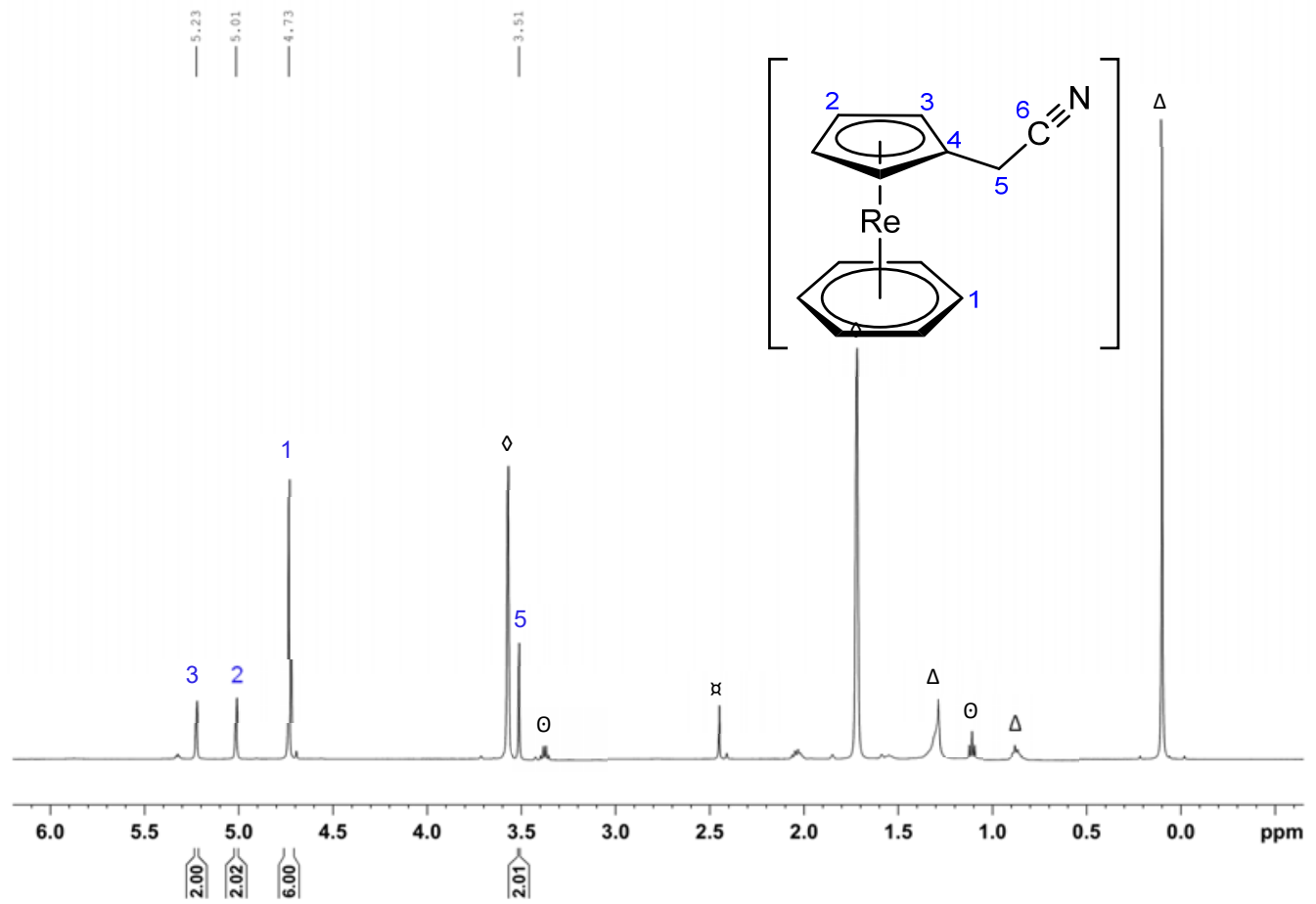

Figure S22. ${ }^{1} \mathrm{H}$ NMR spectrum of $\left[\mathrm{Re}\left(\eta^{5}-\mathrm{C}_{5} \mathrm{H}_{4} \mathrm{CH}_{2} \mathrm{CN}\right)\left(\eta^{6}-\mathrm{C}_{6} \mathrm{H}_{6}\right)\right](6)\left(500 \mathrm{MHz}, \mathrm{C}_{4} \mathrm{D}_{8} \mathrm{O}\right)$. ( $\left(\odot: \mathrm{Et}_{2} \mathrm{O}\right.$ signals).

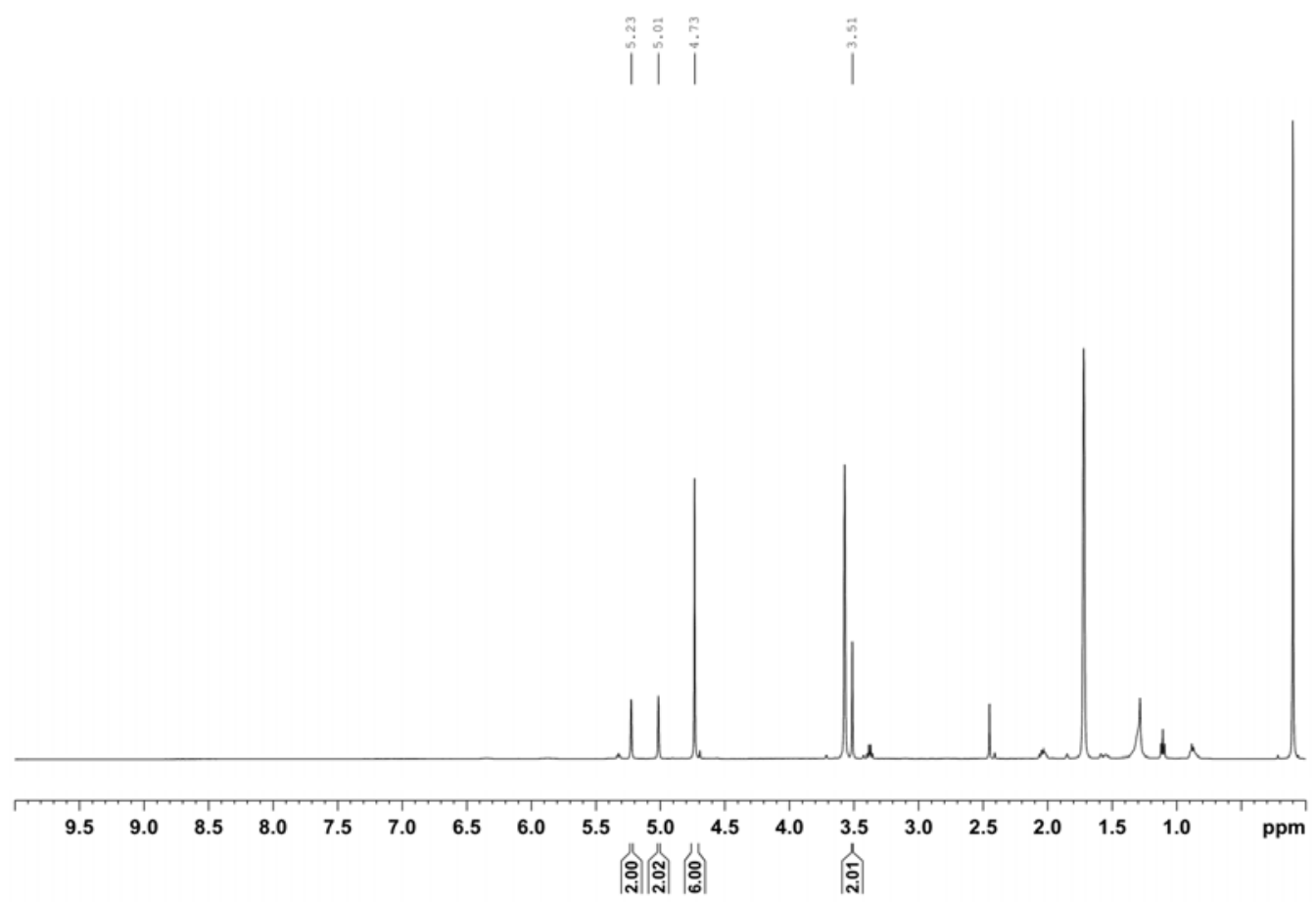

Figure S23. Full ${ }^{1} \mathrm{H}$ NMR spectrum of $\left[\mathrm{Re}\left(\eta^{5}-\mathrm{C}_{5} \mathrm{H}_{4} \mathrm{CH}_{2} \mathrm{CN}\right)\left(\eta^{6}-\mathrm{C}_{6} \mathrm{H}_{6}\right)\right]$ (6) (500 MHz, $\left.\mathrm{C}_{4} \mathrm{D}_{8} \mathrm{O}\right)$. ( $\left(\odot: \mathrm{Et}_{2} \mathrm{O}\right.$ signals). 


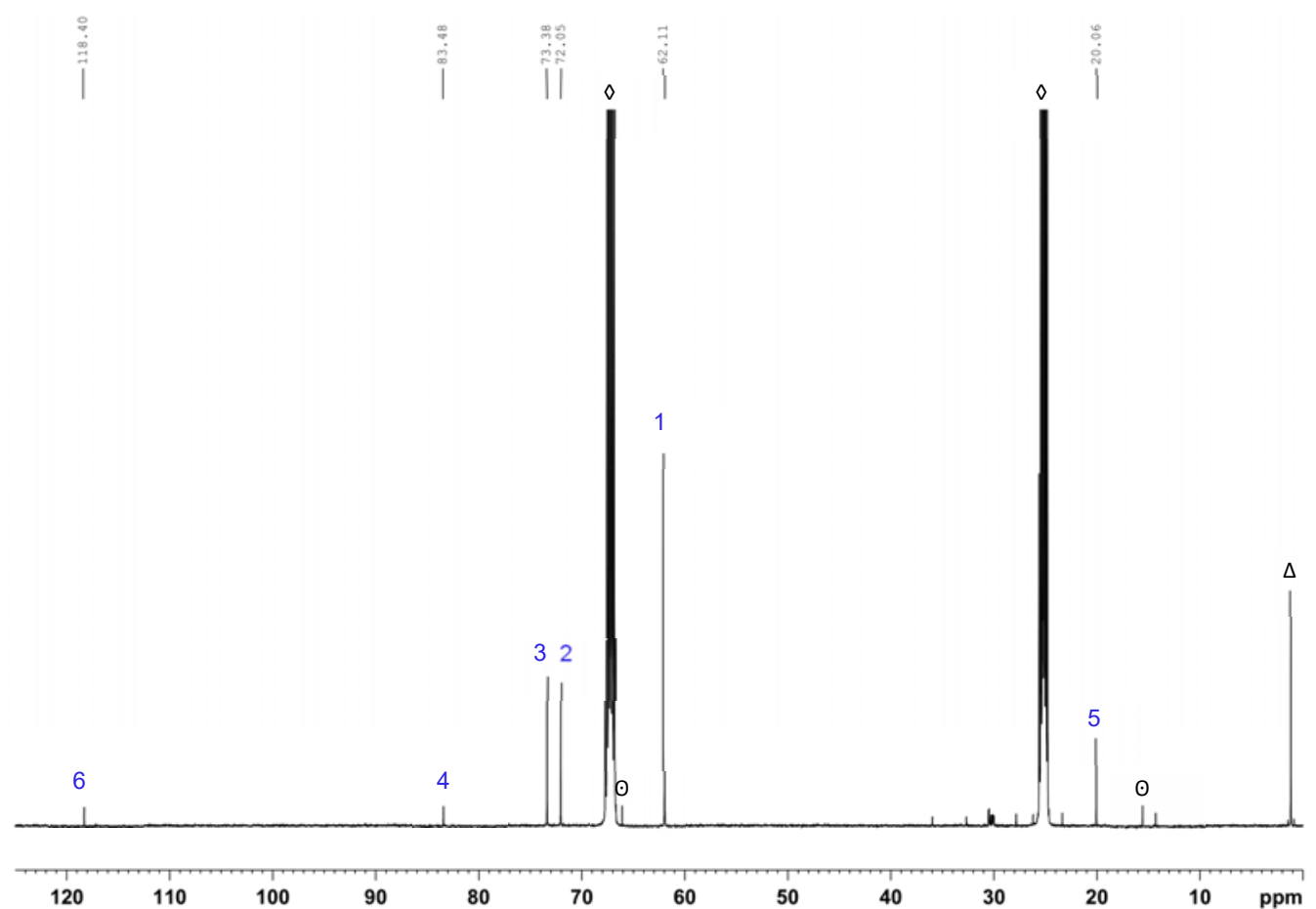

Figure S24. ${ }^{13} \mathrm{C}$ NMR spectrum of $\left[\mathrm{Re}\left(\eta^{5}-\mathrm{C}_{5} \mathrm{H}_{4} \mathrm{CH}_{2} \mathrm{CN}\right)\left(\eta^{6}-\mathrm{C}_{6} \mathrm{H}_{6}\right)\right](6)\left(125 \mathrm{MHz}, \mathrm{C}_{4} \mathrm{D}_{8} \mathrm{O}\right)$. (๑: $\mathrm{Et}_{2} \mathrm{O}$ signals).

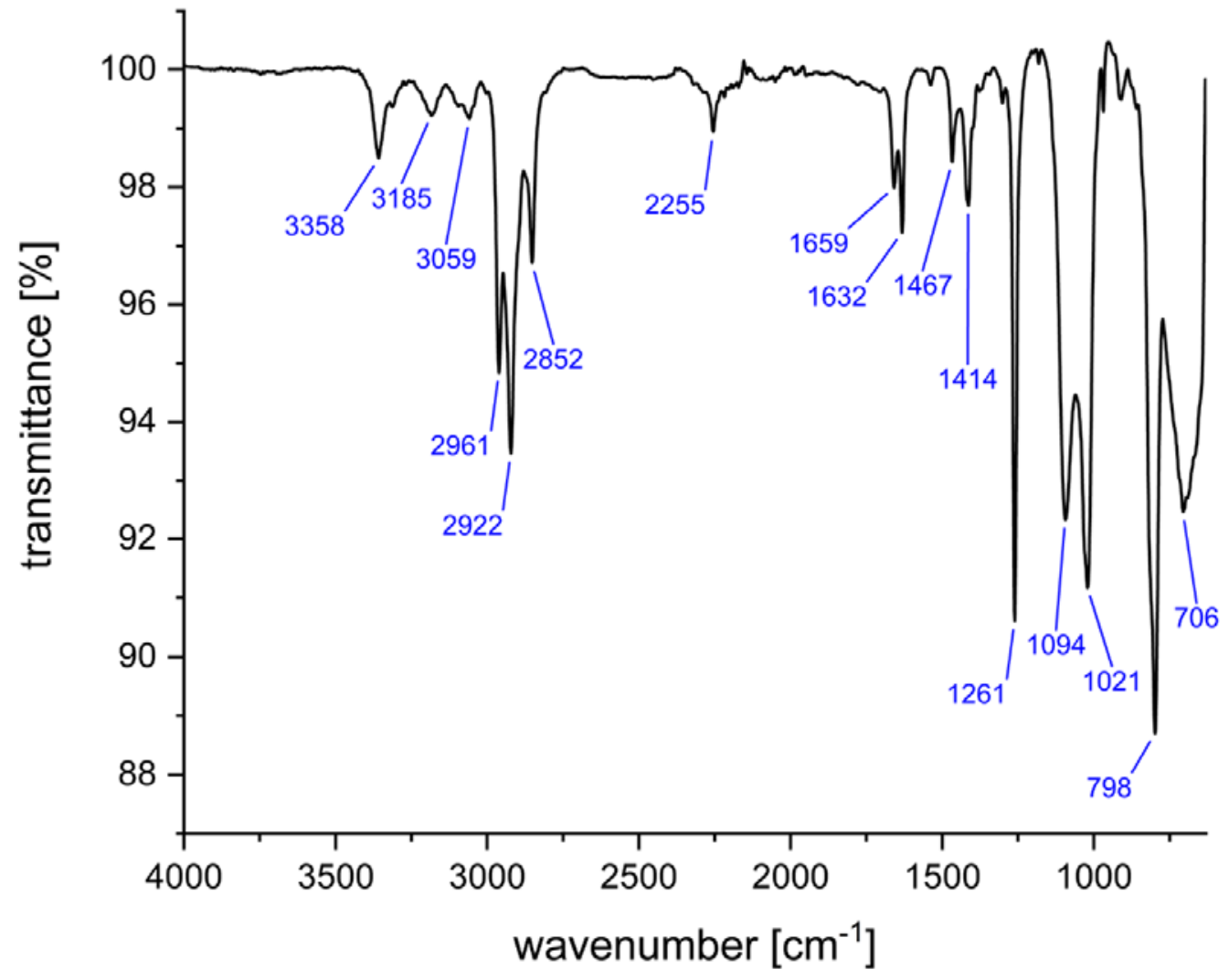

Figure S25. IR spectrum of $\left[\mathrm{Re}\left(\eta^{5}-\mathrm{C}_{5} \mathrm{H}_{4} \mathrm{CH}_{2} \mathrm{CN}\right)\left(\eta^{6}-\mathrm{C}_{6} \mathrm{H}_{6}\right)\right](6)$ (neat). 


\section{$3.6\left[\operatorname{Re}\left(\eta^{5}-\mathrm{C}_{5} \mathrm{H}_{4} \mathrm{CH}_{2} \mathrm{NEt}_{2}\right)\left(\eta^{6}-\mathrm{C}_{6} \mathrm{H}_{6}\right)\right](7)$}

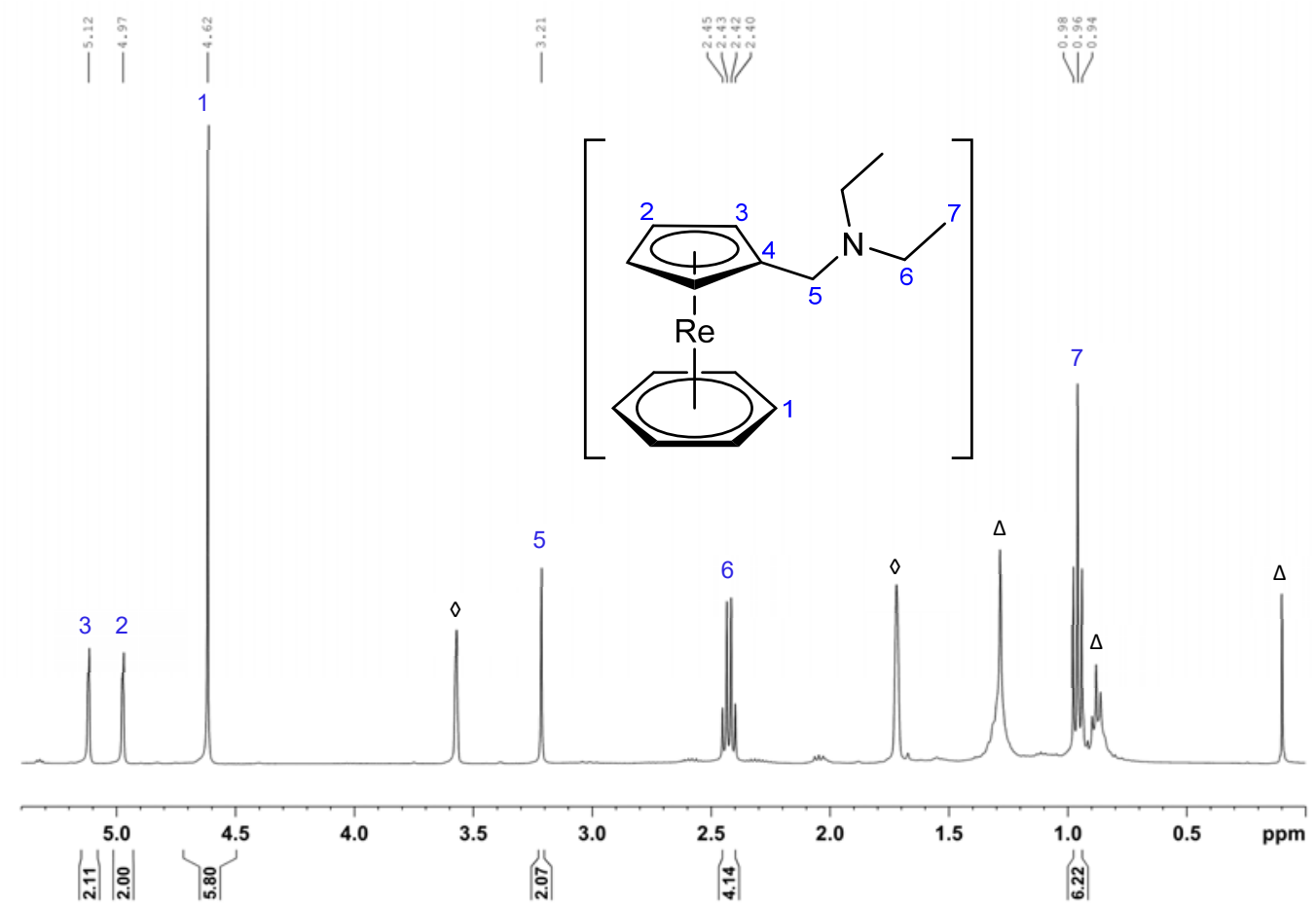

Figure S26. ${ }^{1} \mathrm{H}$ NMR spectrum of $\left[\mathrm{Re}\left(\eta^{5}-\mathrm{C}_{5} \mathrm{H}_{4} \mathrm{CH}_{2} \mathrm{NEt}_{2}\right)\left(\eta^{6}-\mathrm{C}_{6} \mathrm{H}_{6}\right)\right](7)\left(400 \mathrm{MHz}, \mathrm{C}_{4} \mathrm{D}_{8} \mathrm{O}\right)$.

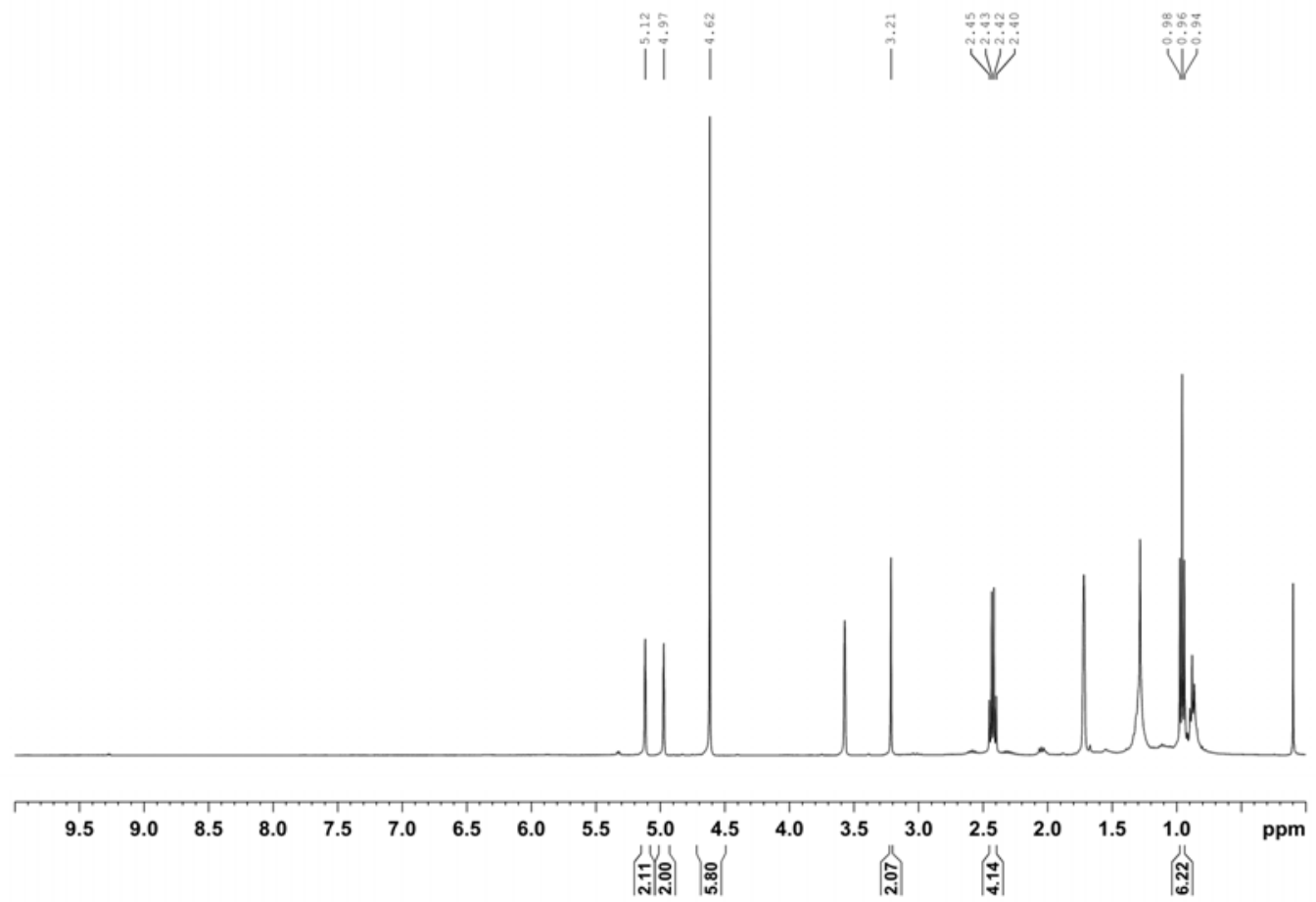

Figure S27. Full ${ }^{1} \mathrm{H}$ NMR spectrum of $\left[\mathrm{Re}\left(\eta^{5}-\mathrm{C}_{5} \mathrm{H}_{4} \mathrm{CH}_{2} \mathrm{NEt}_{2}\right)\left(\eta^{6}-\mathrm{C}_{6} \mathrm{H}_{6}\right)\right](7)\left(400 \mathrm{MHz}, \mathrm{C}_{4} \mathrm{D}_{8} \mathrm{O}\right)$. 


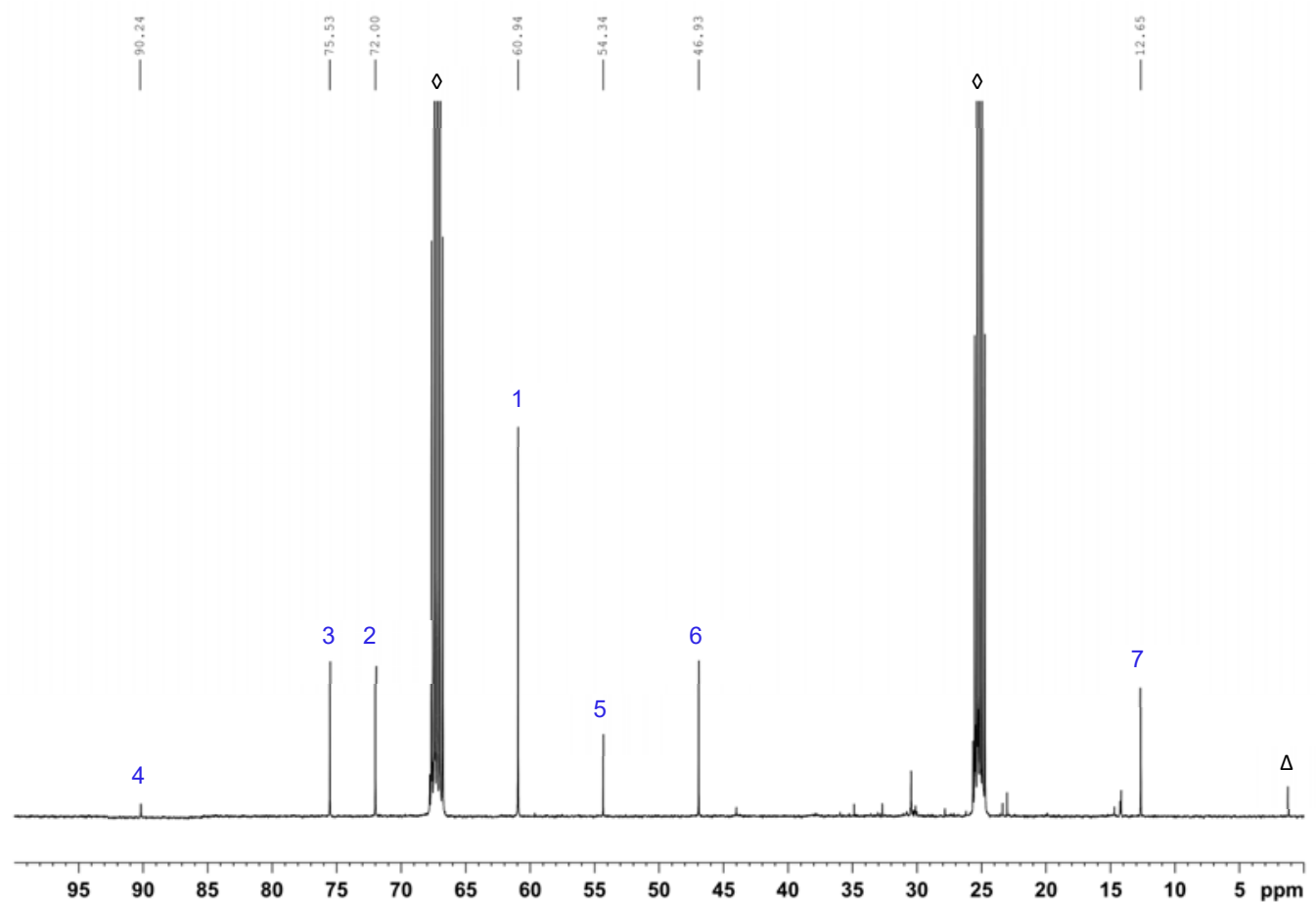

Figure S28. ${ }^{13} \mathrm{C}$ NMR spectrum of $\left[\mathrm{Re}\left(\eta^{5}-\mathrm{C}_{5} \mathrm{H}_{4} \mathrm{CH}_{2} \mathrm{NEt}_{2}\right)\left(\eta^{6}-\mathrm{C}_{6} \mathrm{H}_{6}\right)\right](7)\left(100 \mathrm{MHz}, \mathrm{C}_{4} \mathrm{D}_{8} \mathrm{O}\right)$.

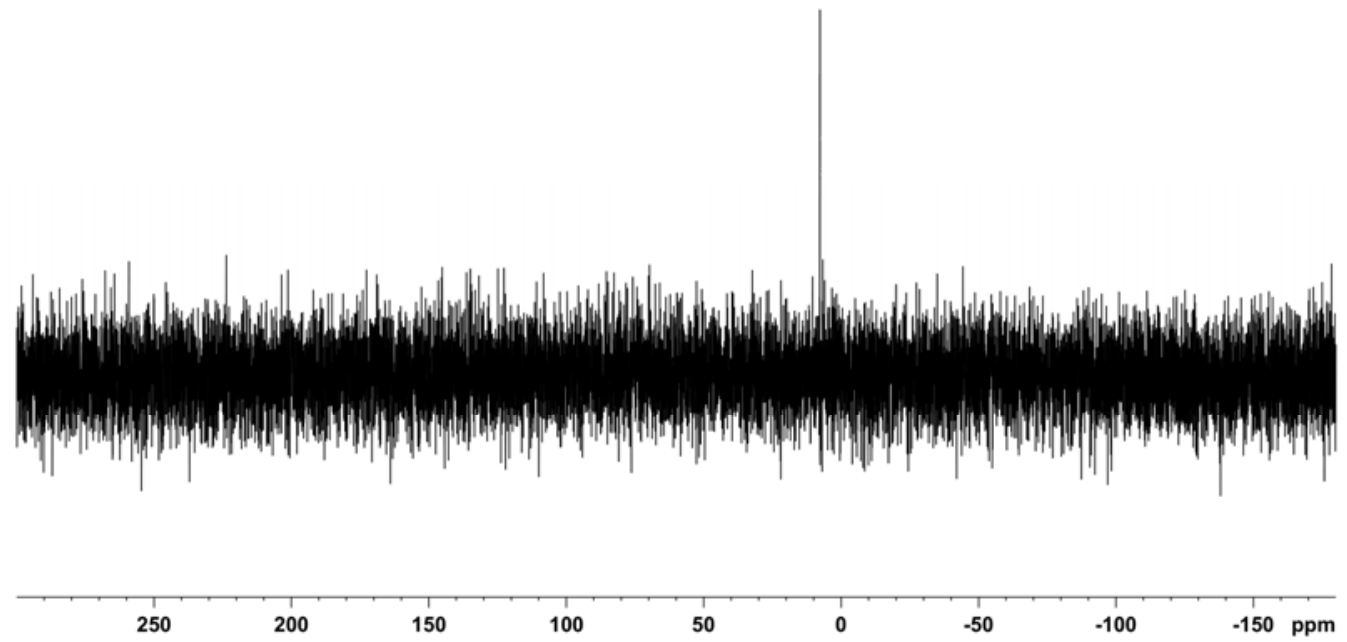

Figure S29. Full ${ }^{15} \mathrm{~N}$ NMR spectrum of $\left[\mathrm{Re}\left(\eta^{5}-\mathrm{C}_{5} \mathrm{H}_{4} \mathrm{CH}_{2} \mathrm{NEt}_{2}\right)\left(\eta^{6}-\mathrm{C}_{6} \mathrm{H}_{6}\right)\right](7)\left(40 \mathrm{MHz}, \mathrm{C}_{4} \mathrm{D}_{8} \mathrm{O}\right)$. 


\section{$3.7\left[\operatorname{Re}\left(\eta^{5}-\mathrm{C}_{5} \mathrm{H}_{4} \mathrm{CH}_{2} \mathrm{OMe}\right)\left(\eta^{6}-\mathrm{C}_{6} \mathrm{H}_{6}\right)\right](8)$}

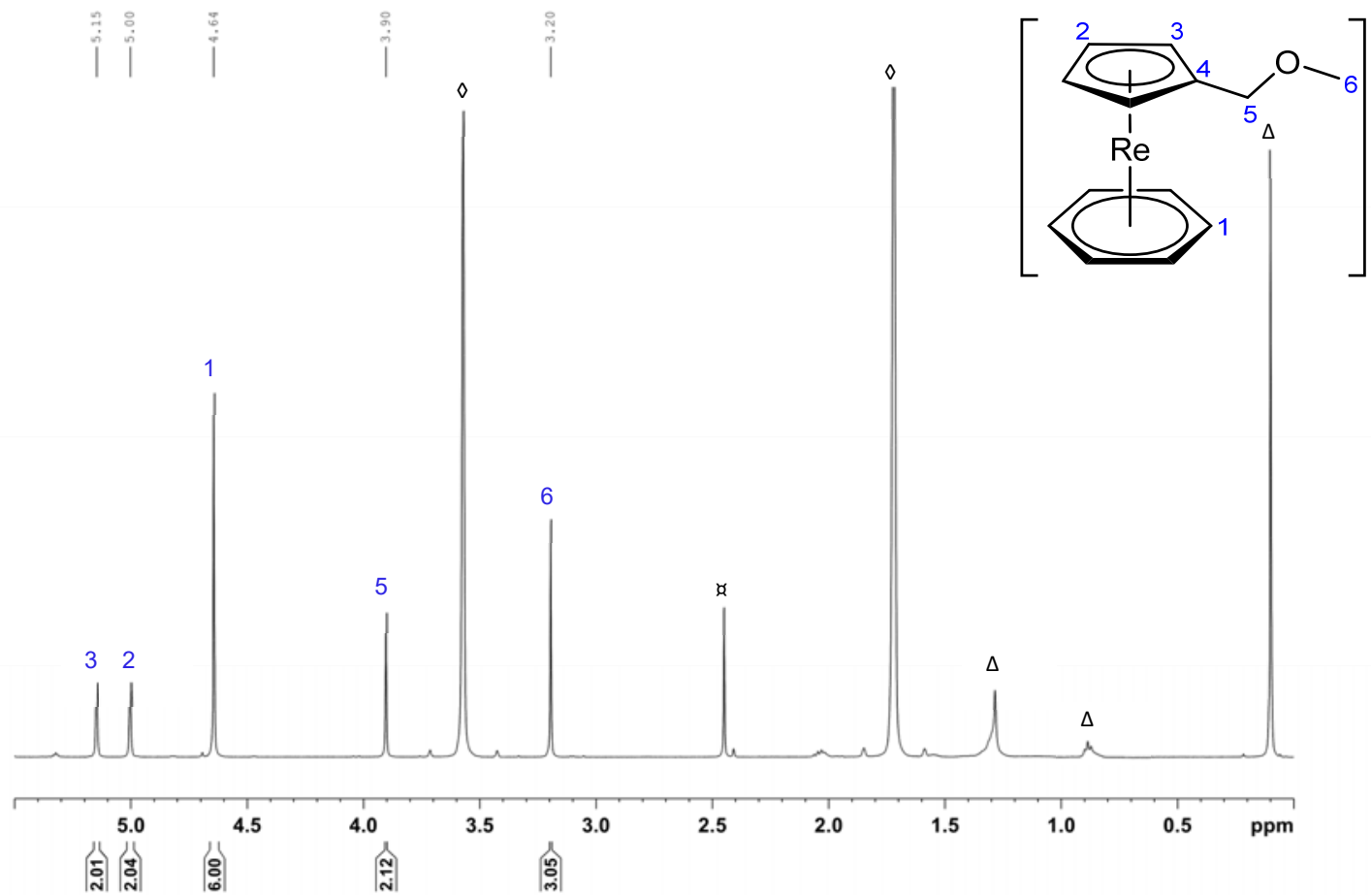

Figure S30. ${ }^{1} \mathrm{H}$ NMR spectrum of $\left[\mathrm{Re}\left(\eta^{5}-\mathrm{C}_{5} \mathrm{H}_{4} \mathrm{CH}_{2} \mathrm{OMe}\right)\left(\eta^{6}-\mathrm{C}_{6} \mathrm{H}_{6}\right)\right](\mathbf{8})\left(500 \mathrm{MHz}, \mathrm{C}_{4} \mathrm{D}_{8} \mathrm{O}\right)$.

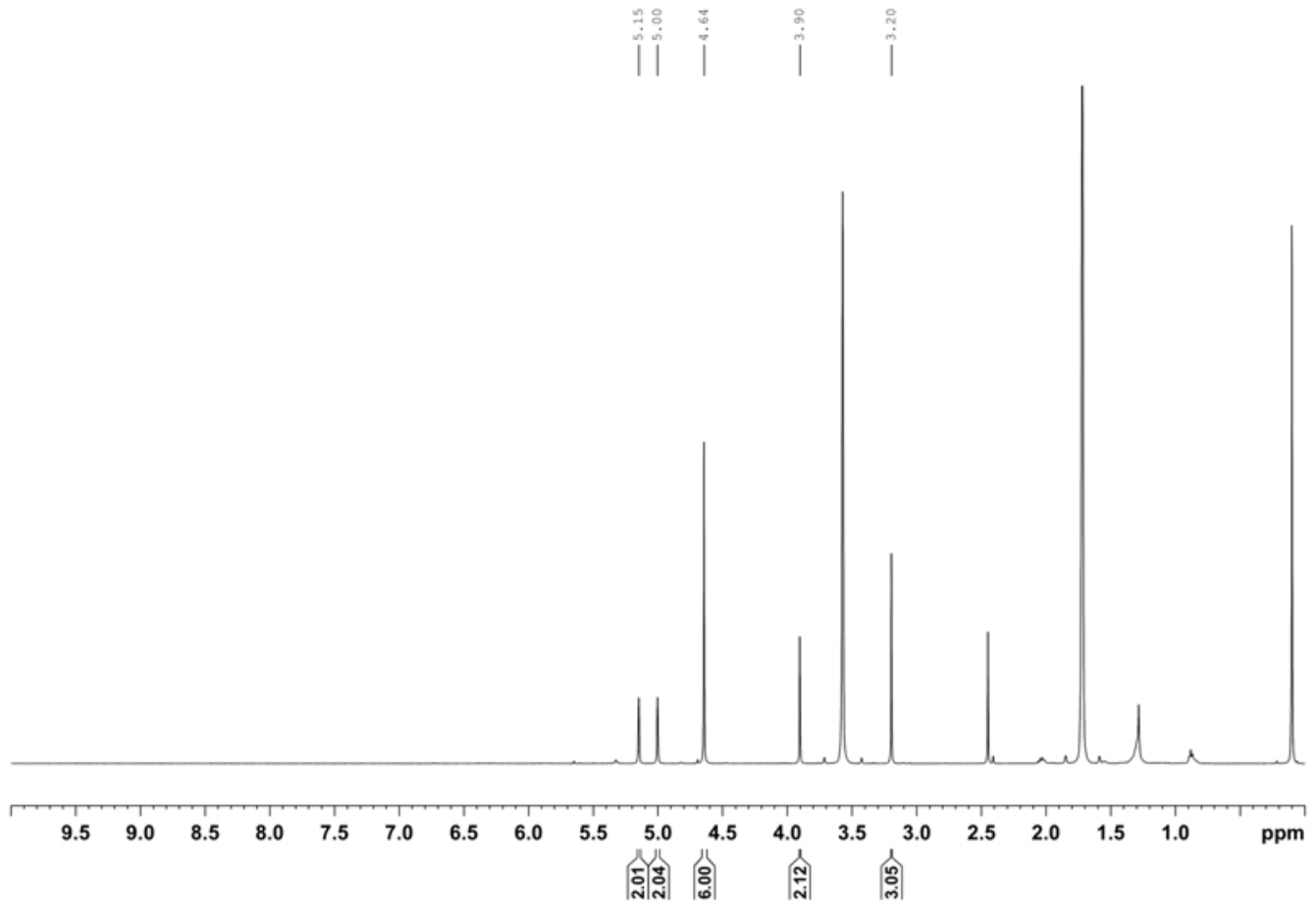

Figure S31. Full ${ }^{1} \mathrm{H}$ NMR spectrum of $\left[\mathrm{Re}\left(\eta^{5}-\mathrm{C}_{5} \mathrm{H}_{4} \mathrm{CH}_{2} \mathrm{OMe}\right)\left(\eta^{6}-\mathrm{C}_{6} \mathrm{H}_{6}\right)\right](8)\left(500 \mathrm{MHz}, \mathrm{C}_{4} \mathrm{D}_{8} \mathrm{O}\right)$. 


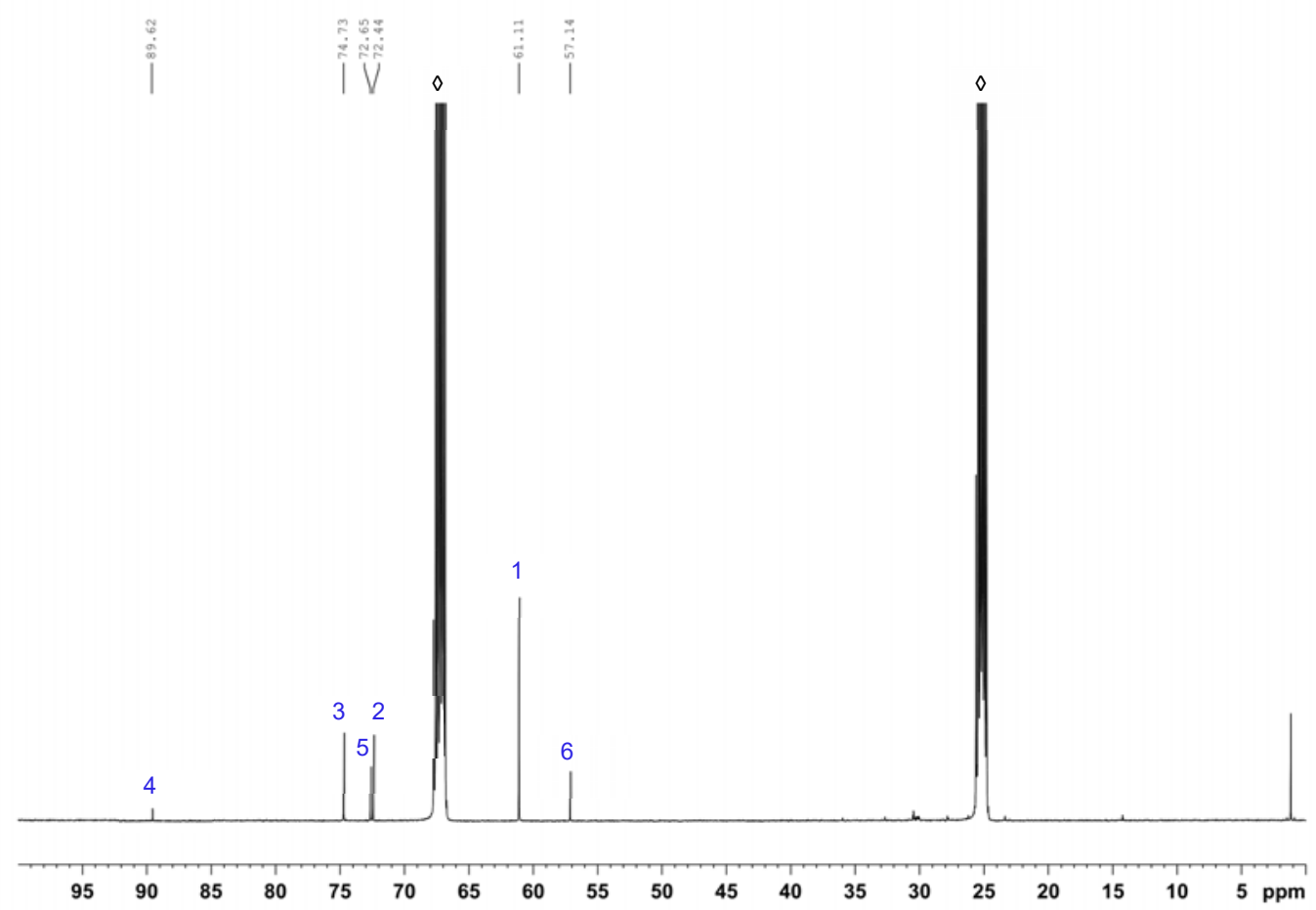

Figure S32. ${ }^{13} \mathrm{C}$ NMR spectrum of $\left[\mathrm{Re}\left(\eta^{5}-\mathrm{C}_{5} \mathrm{H}_{4} \mathrm{CH}_{2} \mathrm{OMe}\right)\left(\eta^{6}-\mathrm{C}_{6} \mathrm{H}_{6}\right)\right](\mathbf{8})\left(125 \mathrm{MHz}, \mathrm{C}_{4} \mathrm{D}_{8} \mathrm{O}\right)$.

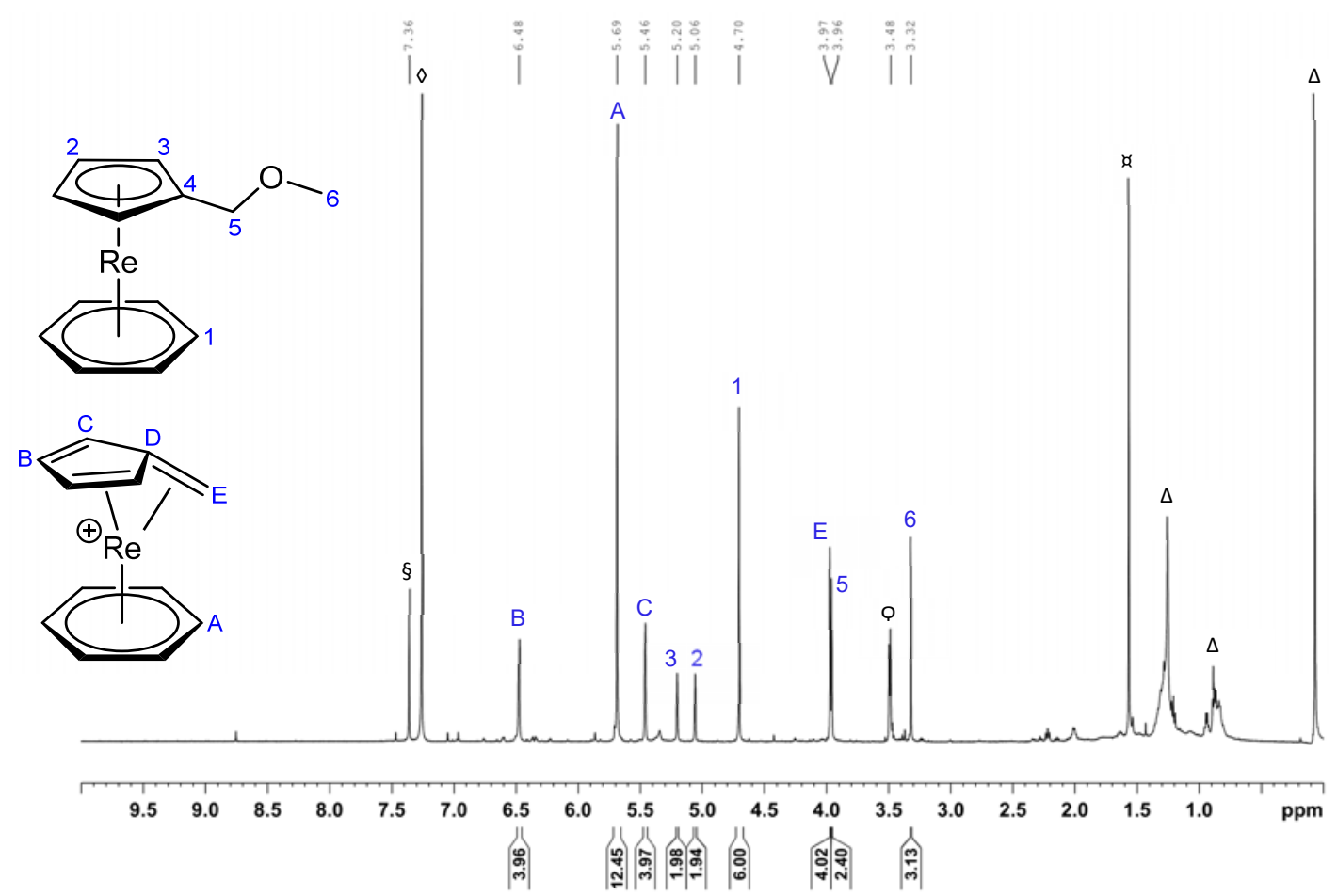

Figure S33. Full ${ }^{1} \mathrm{H}$ NMR spectrum of $\left[\mathrm{Re}\left(\eta^{5}-\mathrm{C}_{5} \mathrm{H}_{4} \mathrm{CH}_{2} \mathrm{OMe}\right)\left(\eta^{6}-\mathrm{C}_{6} \mathrm{H}_{6}\right)\right](8)\left(500 \mathrm{MHz}, \mathrm{CDCl}_{3}\right)$. (§: benzene; Q: $\mathrm{MeOH})$, conducted to observe the instability of complex $\mathbf{8}$ in acidic environment. 


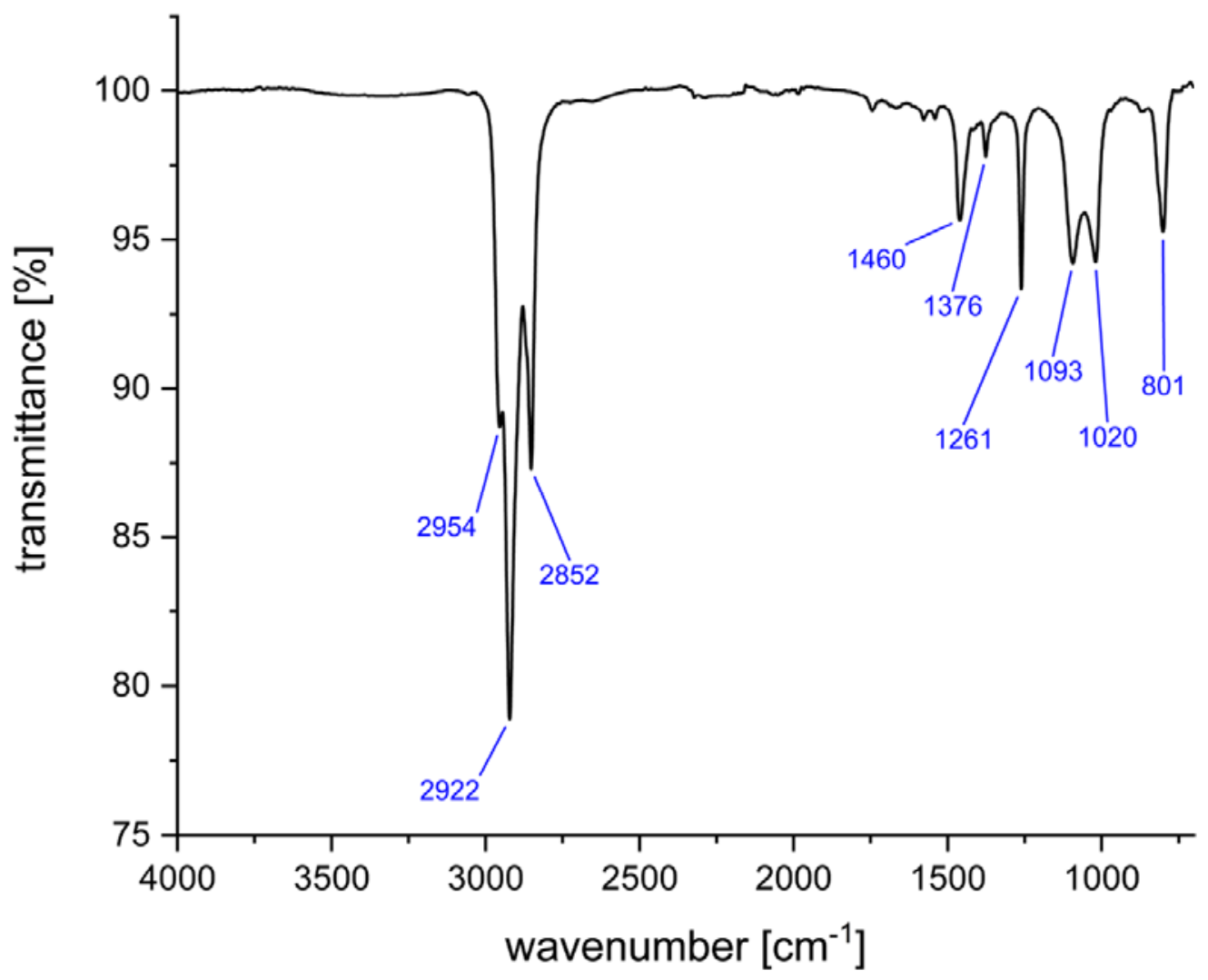

Figure S34. IR spectrum of $\left[\mathrm{Re}\left(\eta^{5}-\mathrm{C}_{5} \mathrm{H}_{4} \mathrm{CH}_{2} \mathrm{OMe}\right)\left(\eta^{6}-\mathrm{C}_{6} \mathrm{H}_{6}\right)\right](8)$ (neat).

\section{$3.8 \quad\left[\operatorname{Re}\left(\eta^{5}-\mathrm{C}_{5} \mathrm{H}_{4} \mathrm{CH}_{2} \mathrm{SEt}\right)\left(\eta^{6}-\mathrm{C}_{6} \mathrm{H}_{6}\right)\right](9)$}

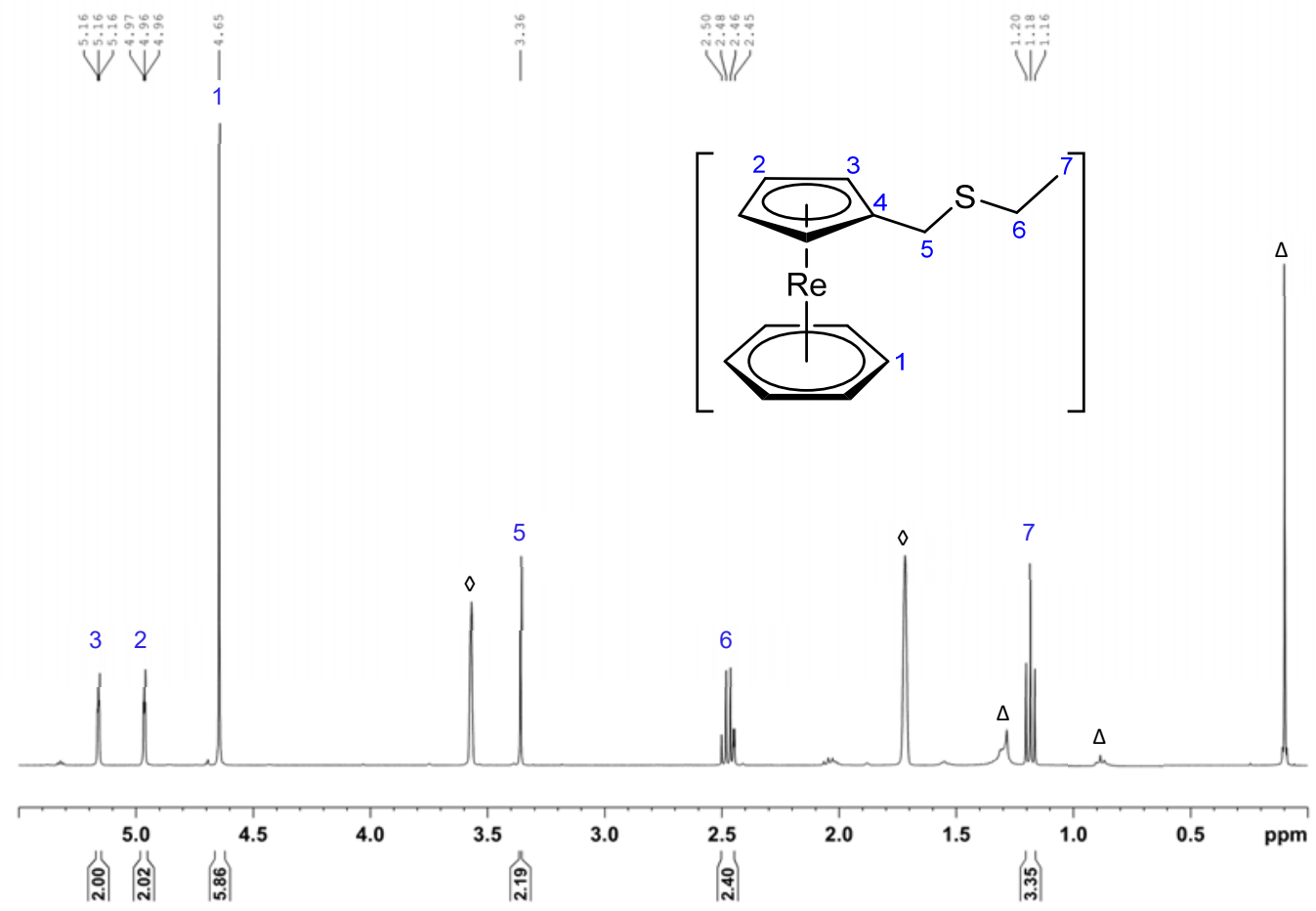

Figure S35. ${ }^{1} \mathrm{H}$ NMR spectrum of $\left[\mathrm{Re}\left(\eta^{5}-\mathrm{C}_{5} \mathrm{H}_{4} \mathrm{CH}_{2} \mathrm{SEt}\right)\left(\eta^{6}-\mathrm{C}_{6} \mathrm{H}_{6}\right)\right](9)\left(400 \mathrm{MHz}, \mathrm{C}_{4} \mathrm{D}_{8} \mathrm{O}\right)$. 


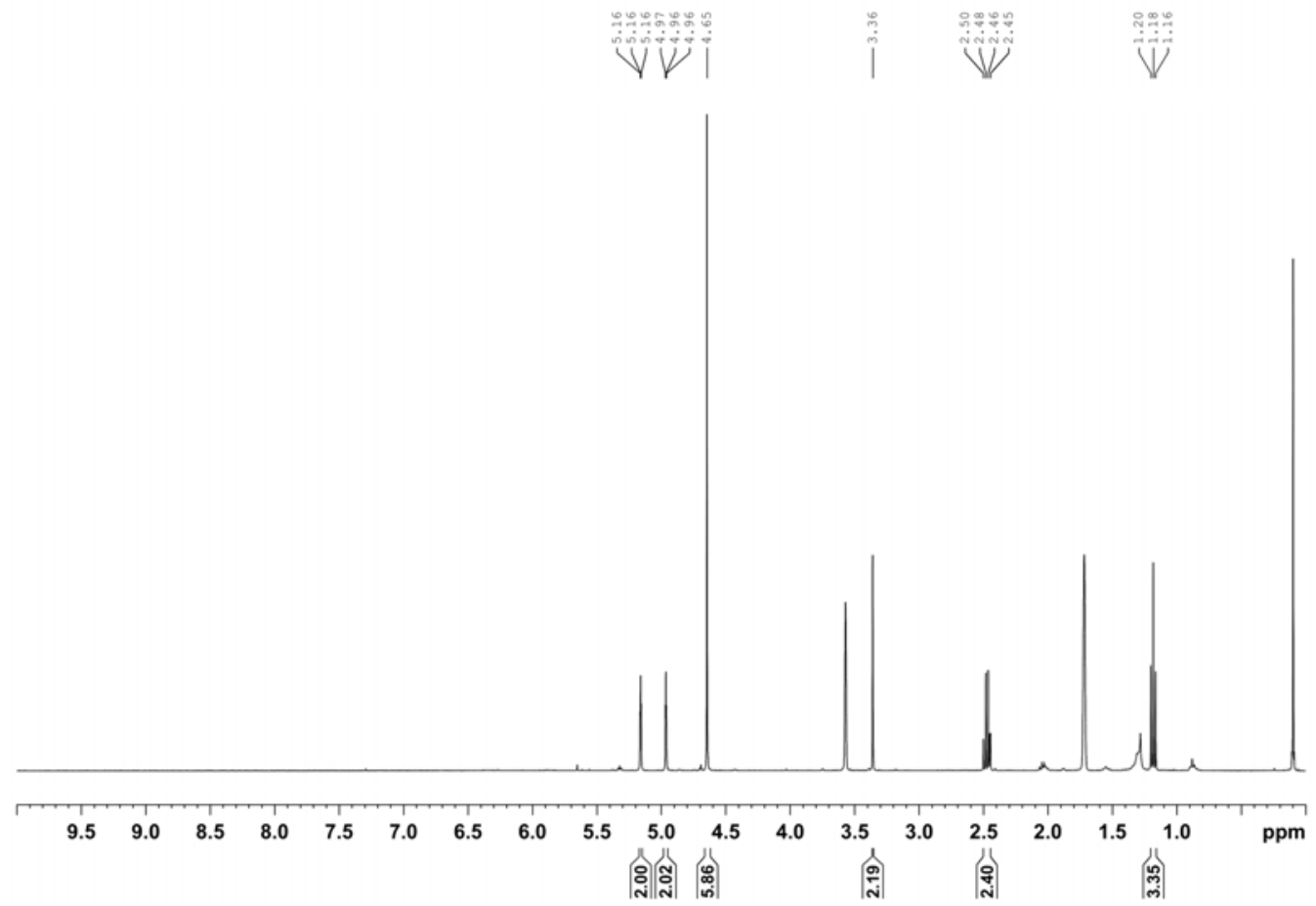

Figure S36. Full ${ }^{1} \mathrm{H}$ NMR spectrum of $\left[\mathrm{Re}\left(\eta^{5}-\mathrm{C}_{5} \mathrm{H}_{4} \mathrm{CH}_{2} \mathrm{SEt}\right)\left(\eta^{6}-\mathrm{C}_{6} \mathrm{H}_{6}\right)\right]$ (9) $\left(400 \mathrm{MHz}, \mathrm{C}_{4} \mathrm{D}_{8} \mathrm{O}\right)$.

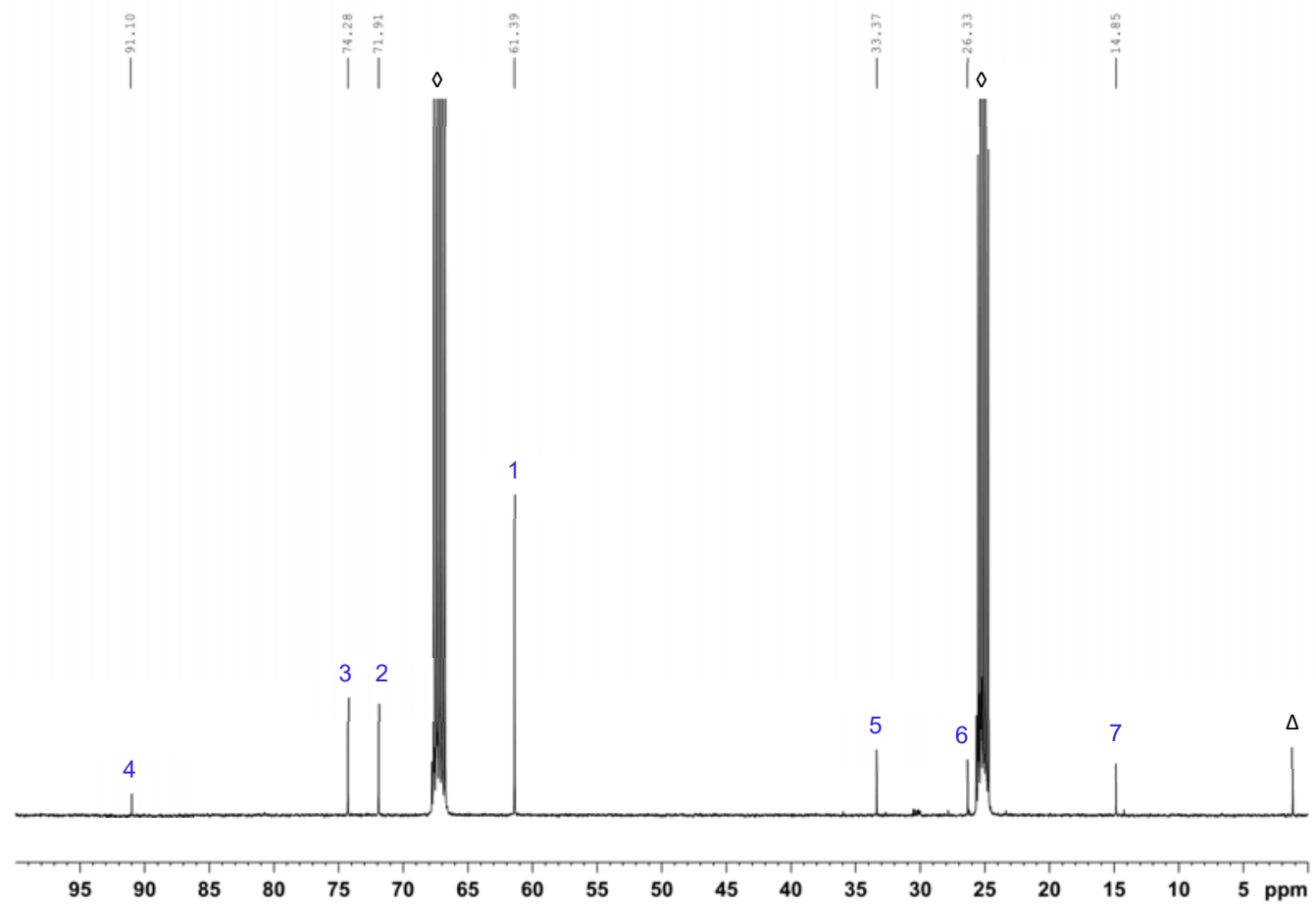

Figure S37. ${ }^{13} \mathrm{C}$ NMR spectrum of $\left[\mathrm{Re}\left(\eta^{5}-\mathrm{C}_{5} \mathrm{H}_{4} \mathrm{CH}_{2} \mathrm{SEt}\right)\left(\eta^{6}-\mathrm{C}_{6} \mathrm{H}_{6}\right)\right](9)\left(100 \mathrm{MHz}, \mathrm{C}_{4} \mathrm{D}_{8} \mathrm{O}\right)$. 


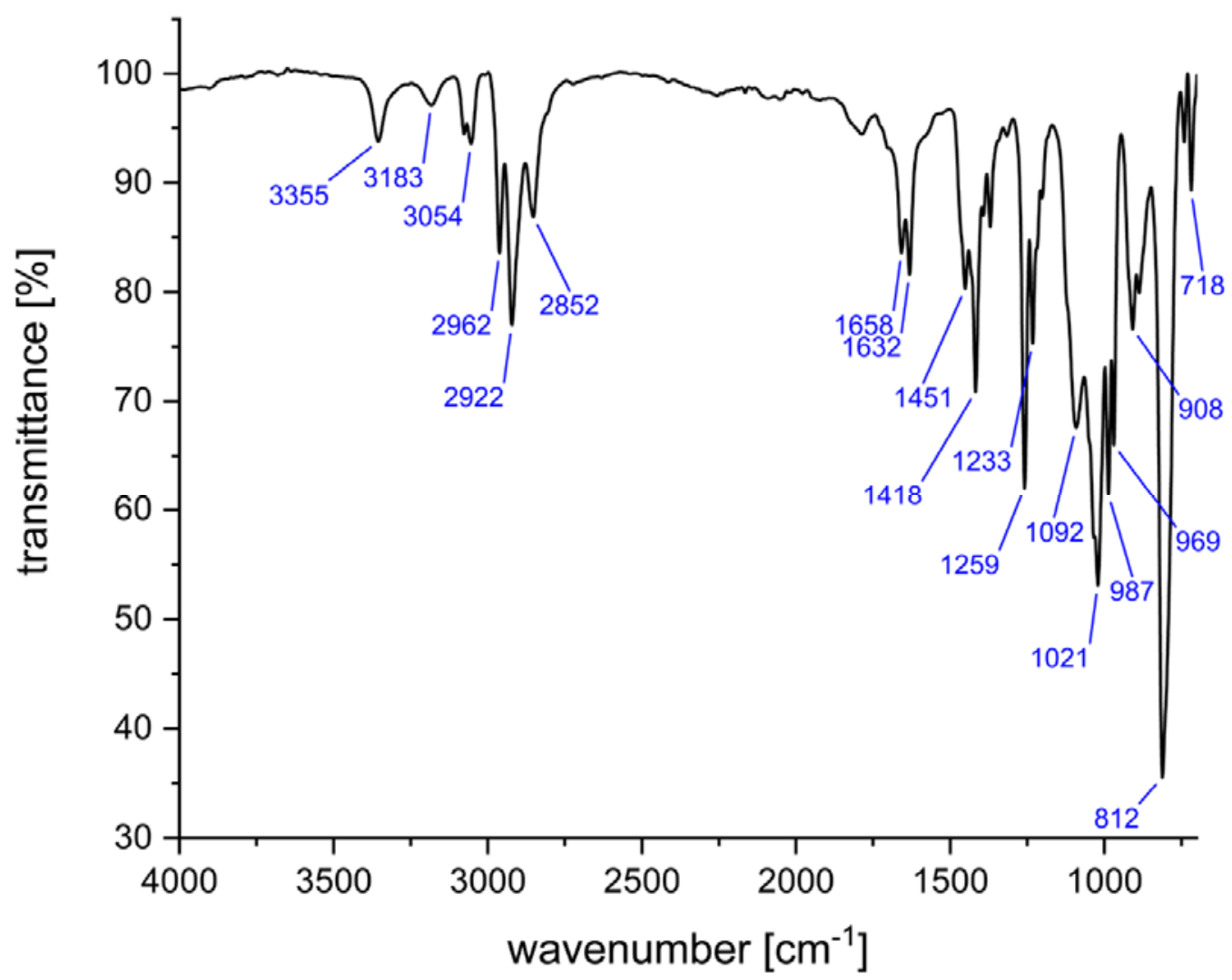

Figure S38. IR spectrum of $\left[\mathrm{Re}\left(\eta^{5}-\mathrm{C}_{5} \mathrm{H}_{4} \mathrm{CH}_{2} \mathrm{SEt}\right)\left(\eta^{6}-\mathrm{C}_{6} \mathrm{H}_{6}\right)\right](9)$ (neat).

\section{$3.9\left[\operatorname{Re}\left(\eta^{5}-\mathrm{C}_{5} \mathrm{H}_{4} \mathrm{CH}_{2} \mathrm{~S}\left(\mathrm{CH}_{2}\right)_{2} \mathrm{OH}\right)\left(\eta^{6}-\mathrm{C}_{6} \mathrm{H}_{6}\right)\right](10)$}

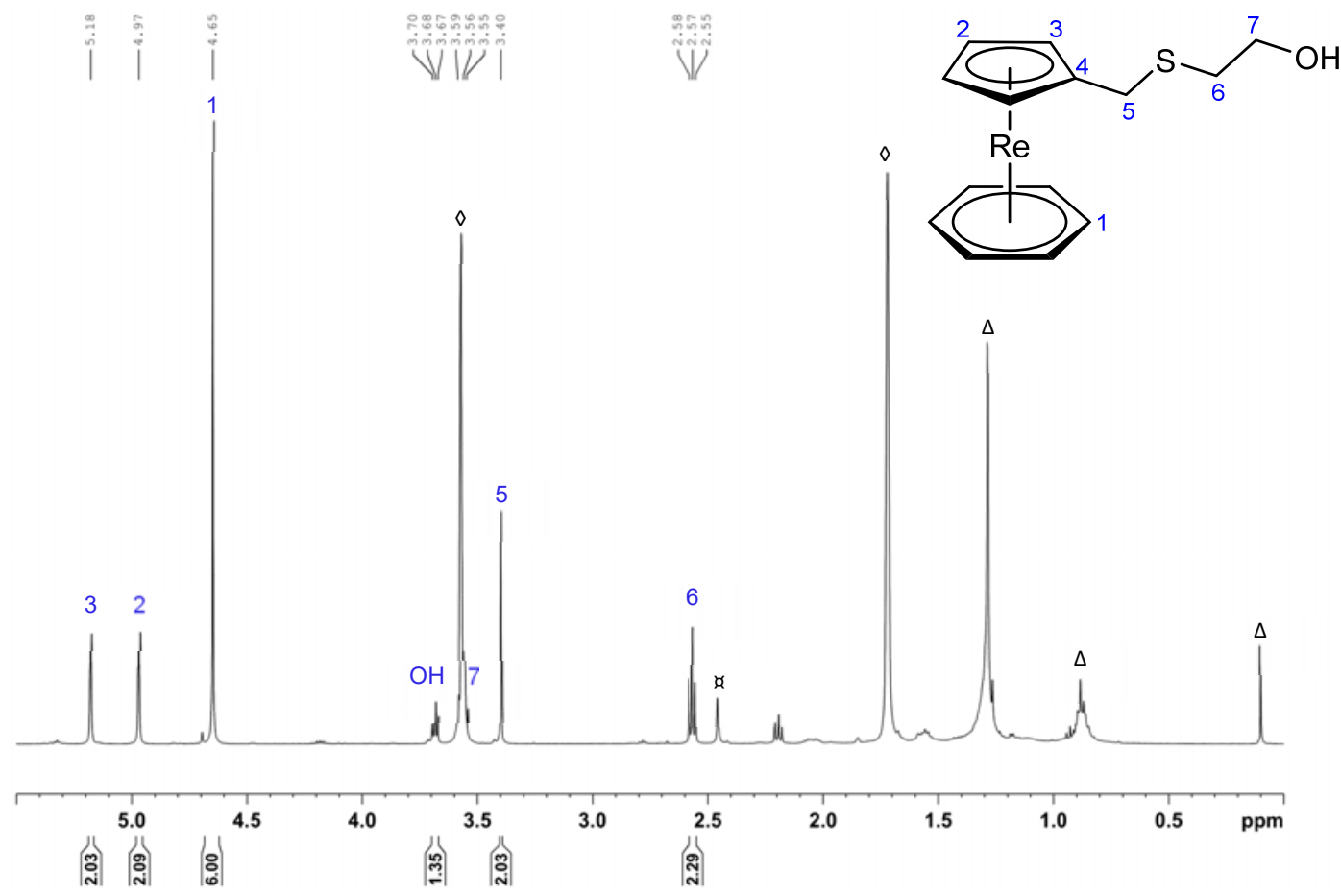

Figure S39. ${ }^{1} \mathrm{H}$ NMR spectrum of $\left[\mathrm{Re}\left(\eta^{5}-\mathrm{C}_{5} \mathrm{H}_{4} \mathrm{CH}_{2} \mathrm{~S}\left(\mathrm{CH}_{2}\right)_{2} \mathrm{OH}\right)\left(\eta^{6}-\mathrm{C}_{6} \mathrm{H}_{6}\right)\right](\mathbf{1 0})\left(500 \mathrm{MHz}, \mathrm{C}_{4} \mathrm{D}_{8} \mathrm{O}\right)$. 


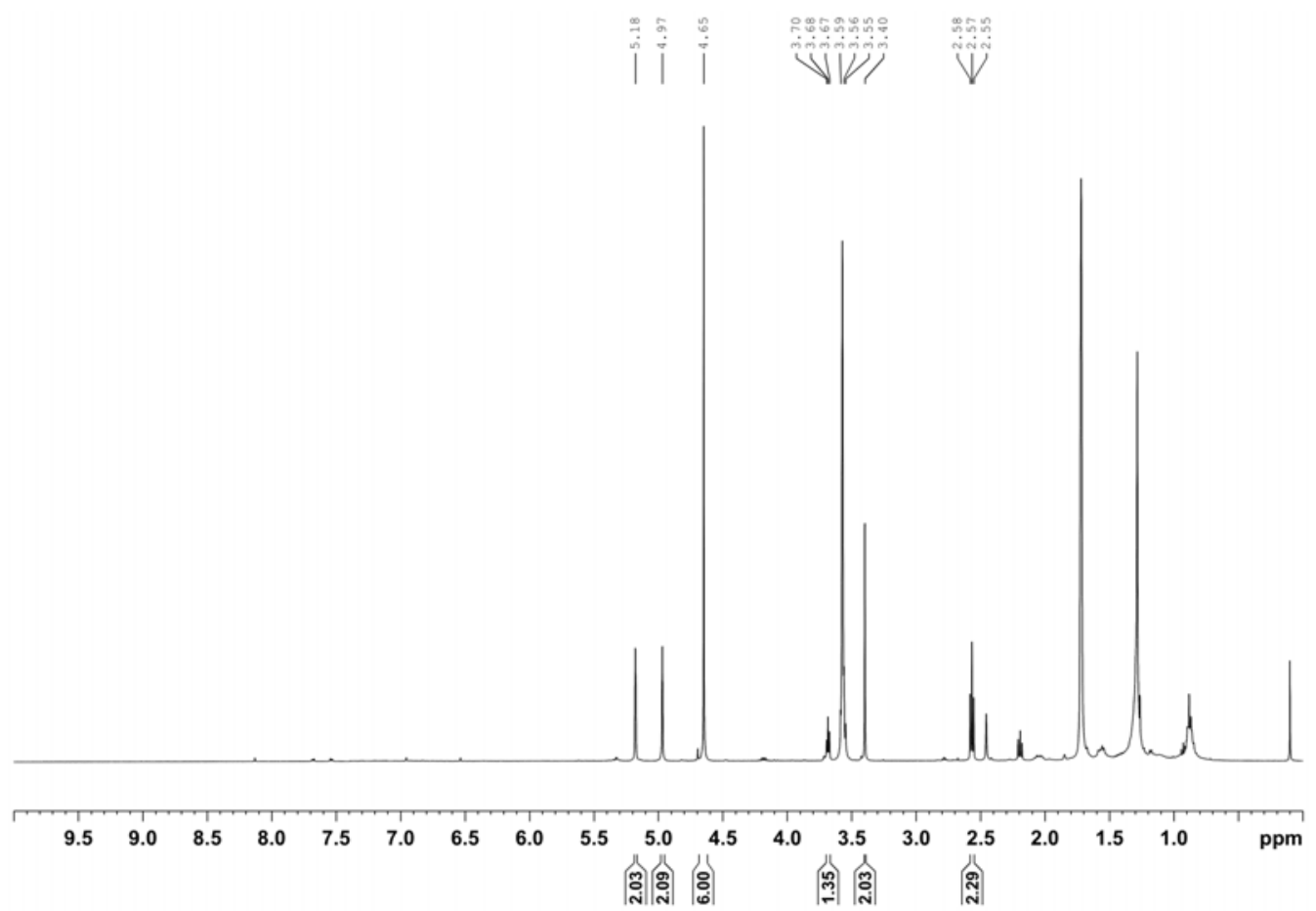

Figure S40. Full ${ }^{1} \mathrm{H}$ NMR spectrum of $\left[\mathrm{Re}\left(\eta^{5}-\mathrm{C}_{5} \mathrm{H}_{4} \mathrm{CH}_{2} \mathrm{~S}\left(\mathrm{CH}_{2}\right)_{2} \mathrm{OH}\right)\left(\eta^{6}-\mathrm{C}_{6} \mathrm{H}_{6}\right)\right](10)\left(500 \mathrm{MHz}, \mathrm{C}_{4} \mathrm{D}_{8} \mathrm{O}\right)$.

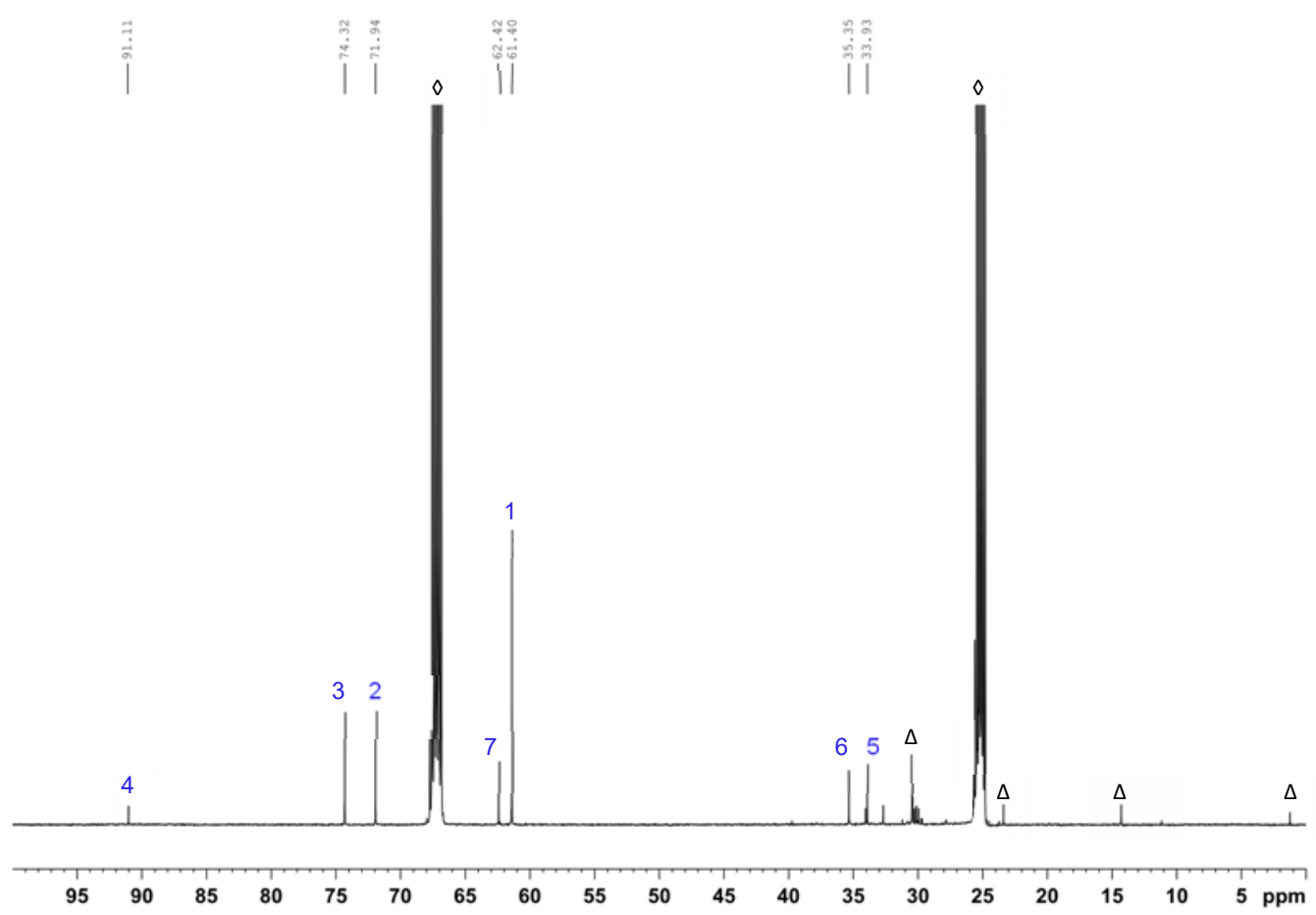

Figure S41. ${ }^{13} \mathrm{C}$ NMR spectrum of $\left[\mathrm{Re}\left(\eta^{5}-\mathrm{C}_{5} \mathrm{H}_{4} \mathrm{CH}_{2} \mathrm{~S}\left(\mathrm{CH}_{2}\right)_{2} \mathrm{OH}\right)\left(\eta^{6}-\mathrm{C}_{6} \mathrm{H}_{6}\right)\right](\mathbf{1 0})\left(125 \mathrm{MHz}, \mathrm{C}_{4} \mathrm{D}_{8} \mathrm{O}\right)$. 


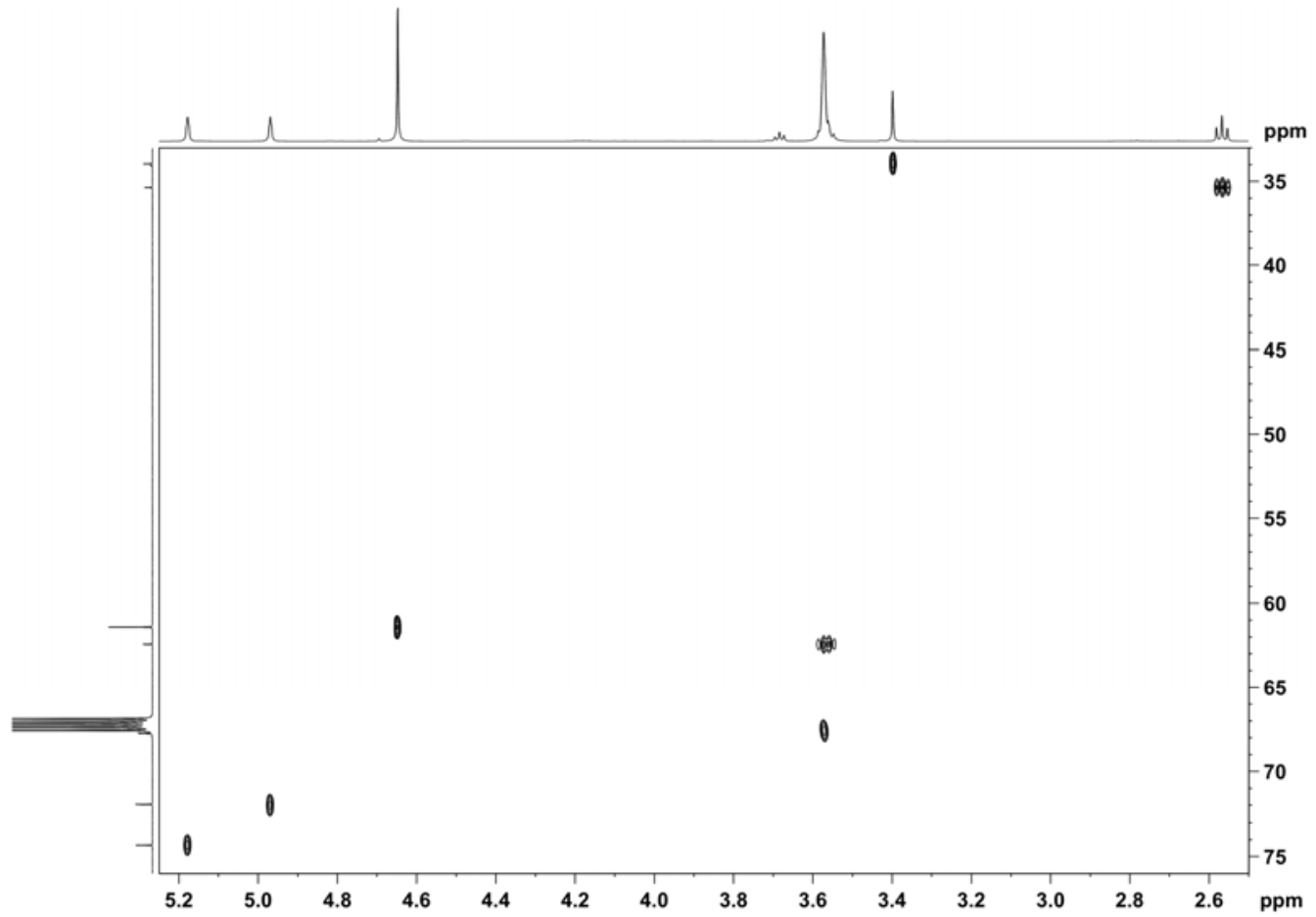

Figure S42. Relevant region in the HSQC spectrum of $\left[\mathrm{Re}\left(\eta^{5}-\mathrm{C}_{5} \mathrm{H}_{4} \mathrm{CH}_{2} \mathrm{~S}\left(\mathrm{CH}_{2}\right)_{2} \mathrm{OH}\right)\left(\eta^{6}-\mathrm{C}_{6} \mathrm{H}_{6}\right)\right]$ (10) $\left(500 / 125 \mathrm{MHz}, \mathrm{C}_{4} \mathrm{D}_{8} \mathrm{O}\right)$.

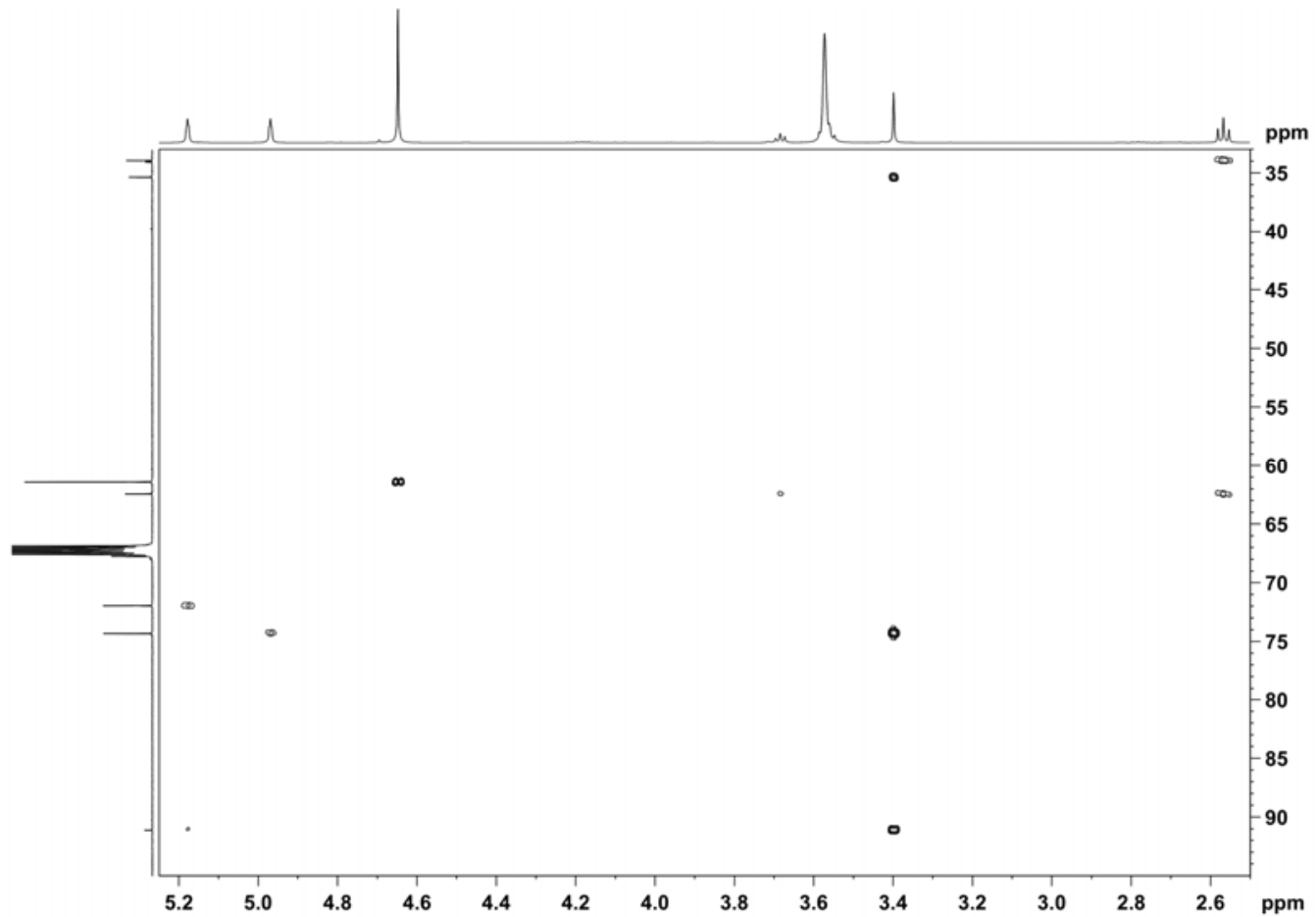

Figure S43. Relevant region in the HMBC spectrum of $\left[\mathrm{Re}\left(\eta^{5}-\mathrm{C}_{5} \mathrm{H}_{4} \mathrm{CH}_{2} \mathrm{~S}\left(\mathrm{CH}_{2}\right)_{2} \mathrm{OH}\right)\left(\eta^{6}-\mathrm{C}_{6} \mathrm{H}_{6}\right)\right](\mathbf{1 0})$ $\left(500 / 125 \mathrm{MHz}, \mathrm{C}_{4} \mathrm{D}_{8} \mathrm{O}\right)$. 


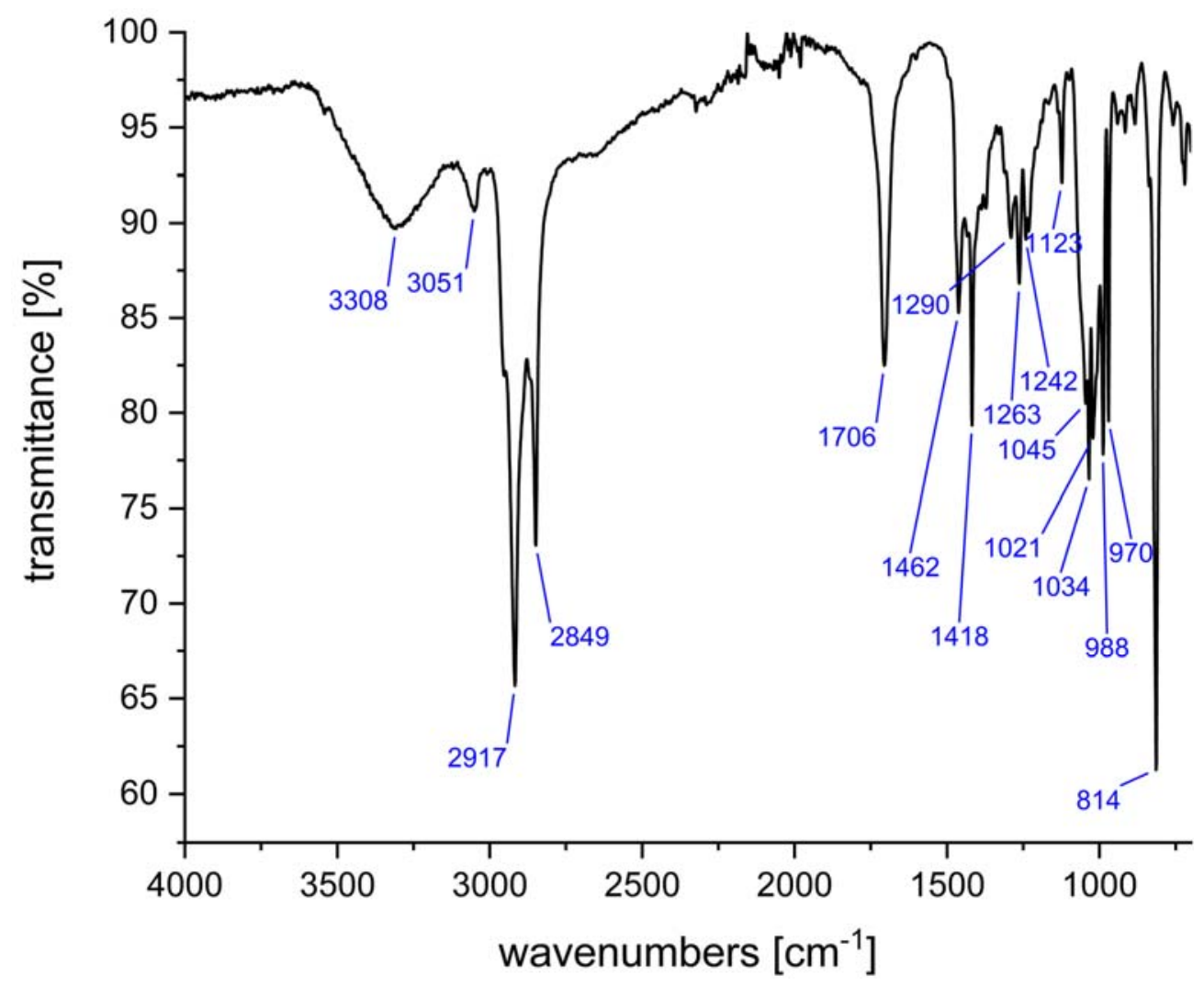

Figure S44. IR spectrum of $\left[\mathrm{Re}\left(\eta^{5}-\mathrm{C}_{5} \mathrm{H}_{4} \mathrm{CH}_{2} \mathrm{~S}\left(\mathrm{CH}_{2}\right)_{2} \mathrm{OH}\right)\left(\eta^{6}-\mathrm{C}_{6} \mathrm{H}_{6}\right)\right](10)$ (neat).

\section{$3.10\left[\operatorname{Re}\left(\eta^{5}-\mathrm{C}_{5} \mathrm{H}_{4} \mathrm{CH}_{2} \mathrm{PTA}\right)\left(\eta^{6}-\mathrm{C}_{6} \mathrm{H}_{6}\right)\right](\mathrm{OTf})([11](\mathrm{OTf}))$}

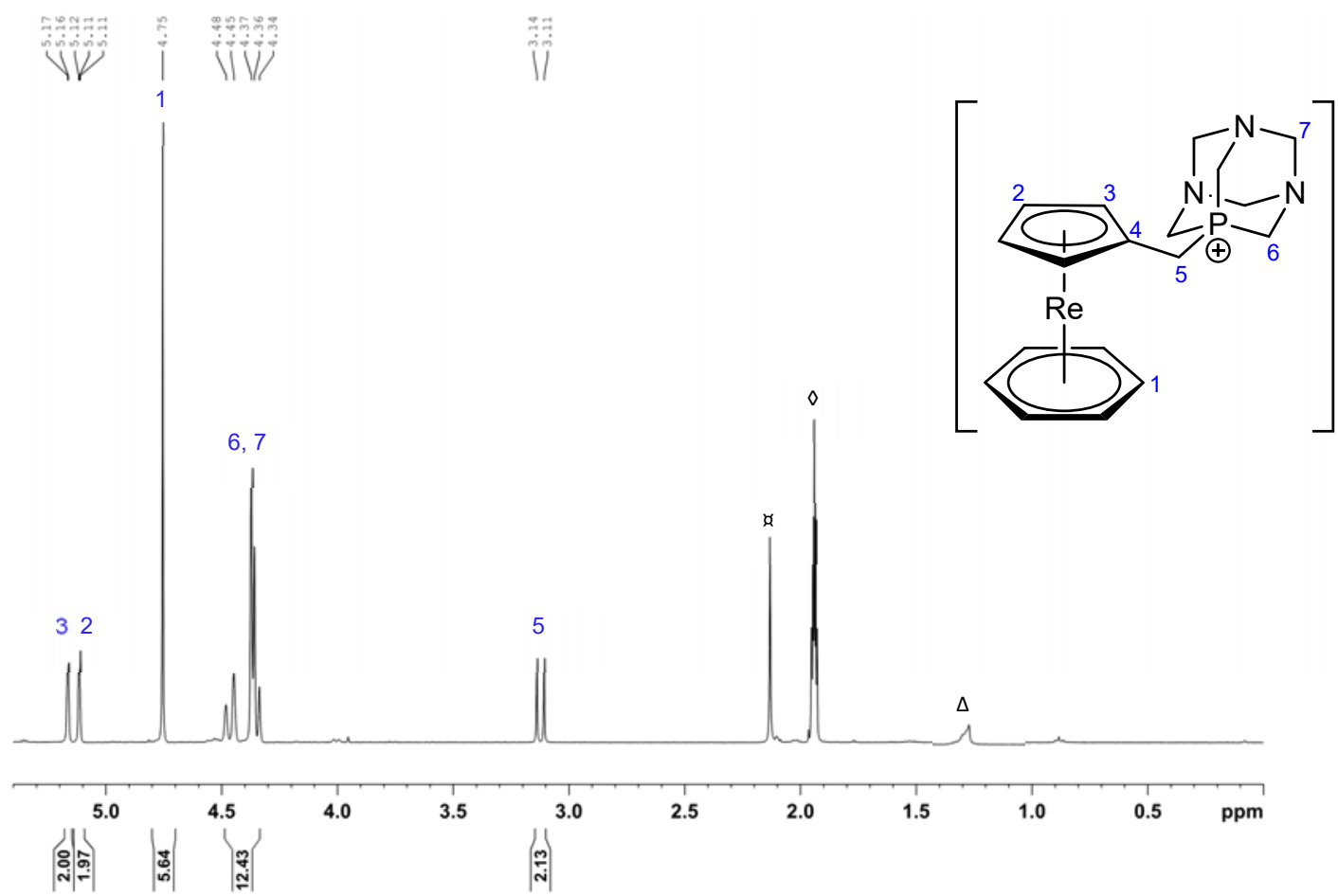

Figure S45. ${ }^{1} \mathrm{H}$ NMR spectrum of $\left[\mathrm{Re}\left(\eta^{5}-\mathrm{C}_{5} \mathrm{H}_{4} \mathrm{CH}_{2} \mathrm{PTA}\right)\left(\eta^{6}-\mathrm{C}_{6} \mathrm{H}_{6}\right)\right](\mathrm{OTf})([11](\mathrm{OTf}))\left(400 \mathrm{MHz}, \mathrm{CD}_{3} \mathrm{CN}\right)$. 


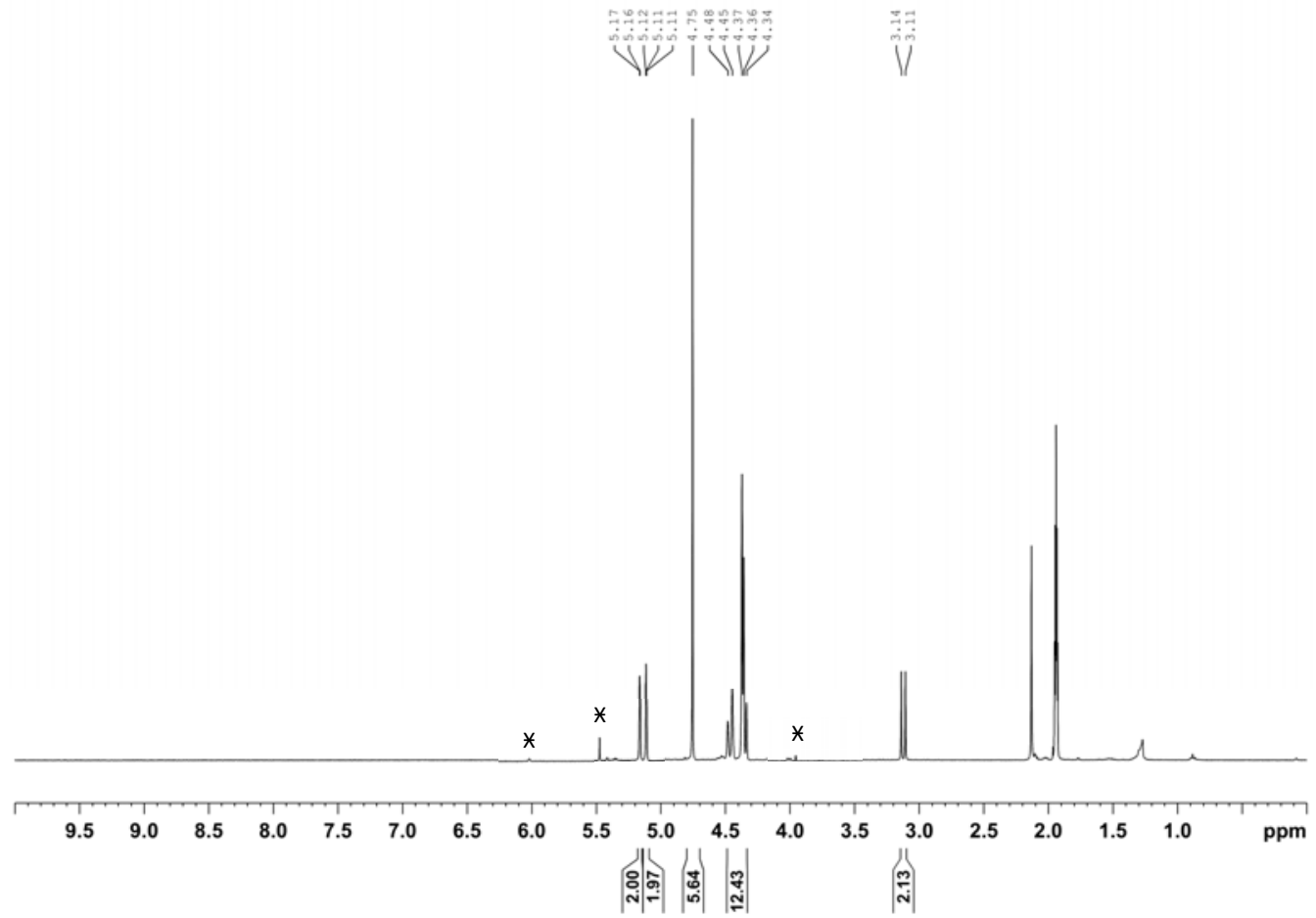

Figure S46. Full ${ }^{1} \mathrm{H}$ NMR spectrum of $\left[\mathrm{Re}\left(\eta^{5}-\mathrm{C}_{5} \mathrm{H}_{4} \mathrm{CH}_{2} \mathrm{PTA}\right)\left(\eta^{6}-\mathrm{C}_{6} \mathrm{H}_{6}\right)\right](\mathrm{OTf})([11](\mathrm{OTf}))\left(400 \mathrm{MHz}, \mathrm{CD}_{3} \mathrm{CN}\right)$. (*: signals of the impurity $\mathbf{3}^{+}$).

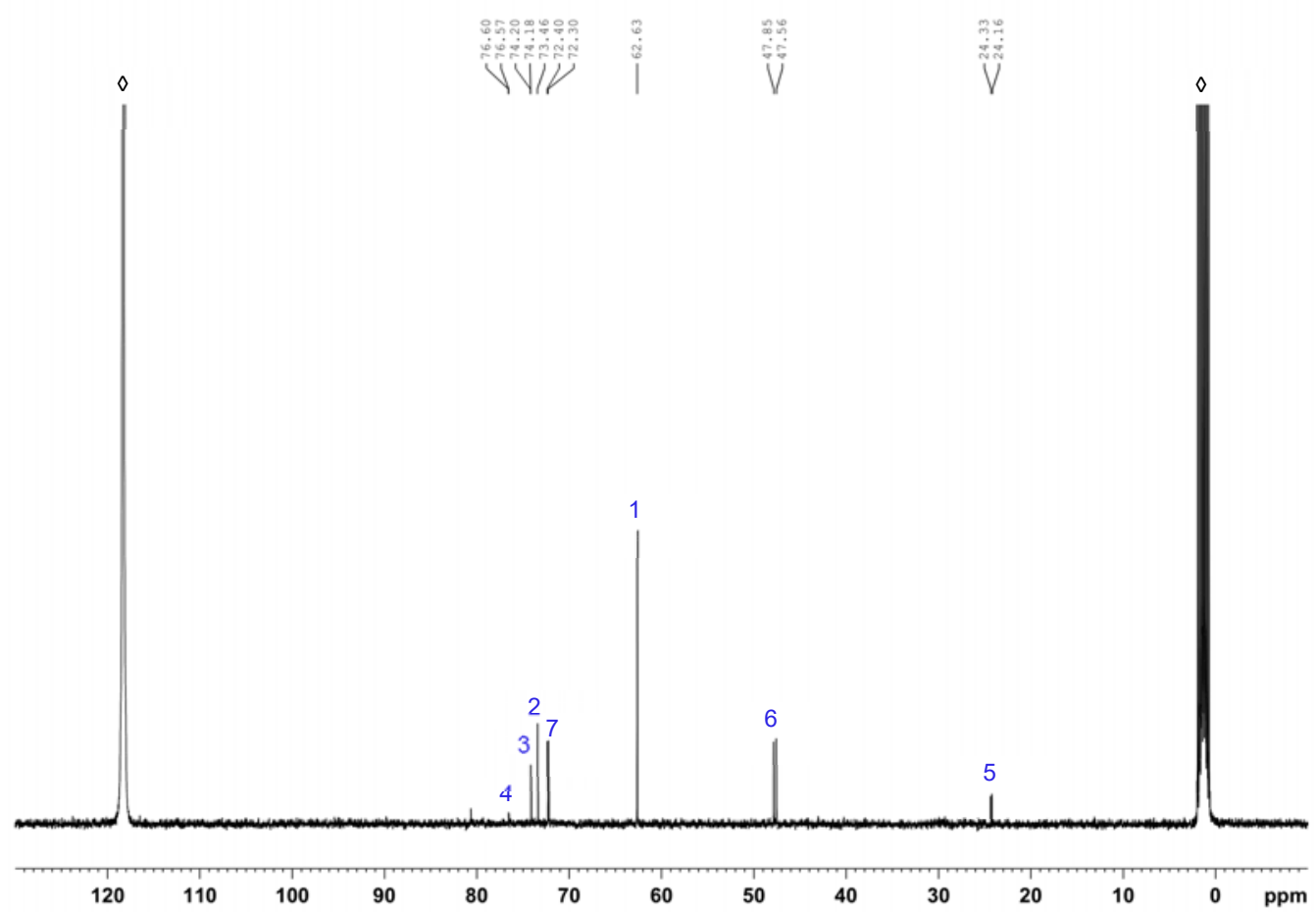

Figure S47. ${ }^{13} \mathrm{C}$ NMR spectrum of $\left[\operatorname{Re}\left(\eta^{5}-\mathrm{C}_{5} \mathrm{H}_{4} \mathrm{CH}_{2} \mathrm{PTA}\right)\left(\eta^{6}-\mathrm{C}_{6} \mathrm{H}_{6}\right)\right](\mathrm{OTf})([11](\mathrm{OTf}))\left(100 \mathrm{MHz}, \mathrm{CD}_{3} \mathrm{CN}\right)$. 


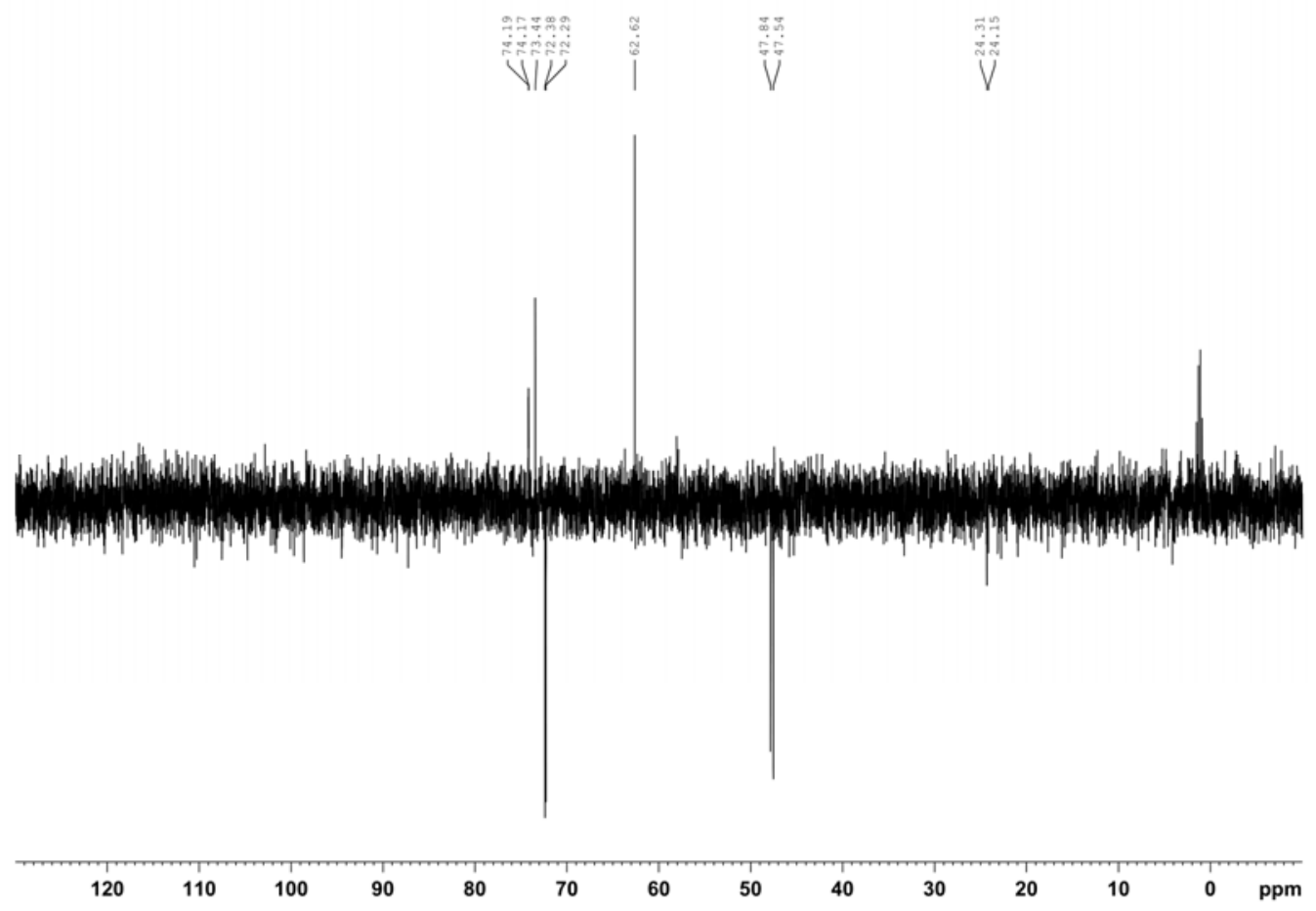

Figure S48. DEPT135 NMR spectrum of $\left[\mathrm{Re}\left(\eta^{5}-\mathrm{C}_{5} \mathrm{H}_{4} \mathrm{CH}_{2} \mathrm{PTA}\right)\left(\eta^{6}-\mathrm{C}_{6} \mathrm{H}_{6}\right)\right](\mathrm{OTf})([11](\mathrm{OTf}))\left(100 \mathrm{MHz}, \mathrm{CD}_{3} \mathrm{CN}\right)$.

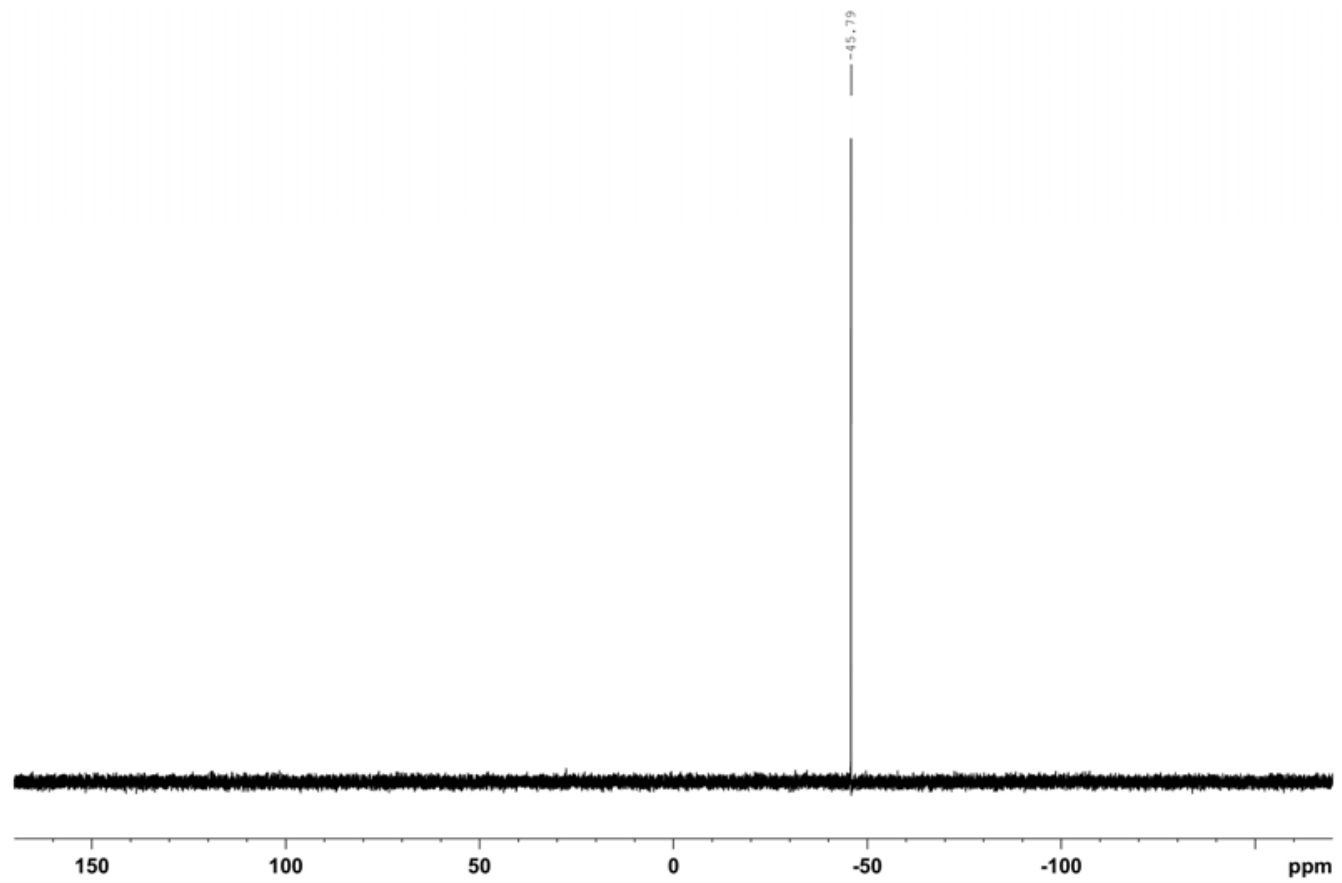

Figure S49. Full ${ }^{31} \mathrm{P}$ NMR spectrum of $\left[\mathrm{Re}\left(\eta^{5}-\mathrm{C}_{5} \mathrm{H}_{4} \mathrm{CH}_{2} \mathrm{PTA}\right)\left(\eta^{6}-\mathrm{C}_{6} \mathrm{H}_{6}\right)\right](\mathrm{OTf})([11](\mathrm{OTf}))\left(162 \mathrm{MHz}, \mathrm{CD}_{3} \mathrm{CN}\right)$. 


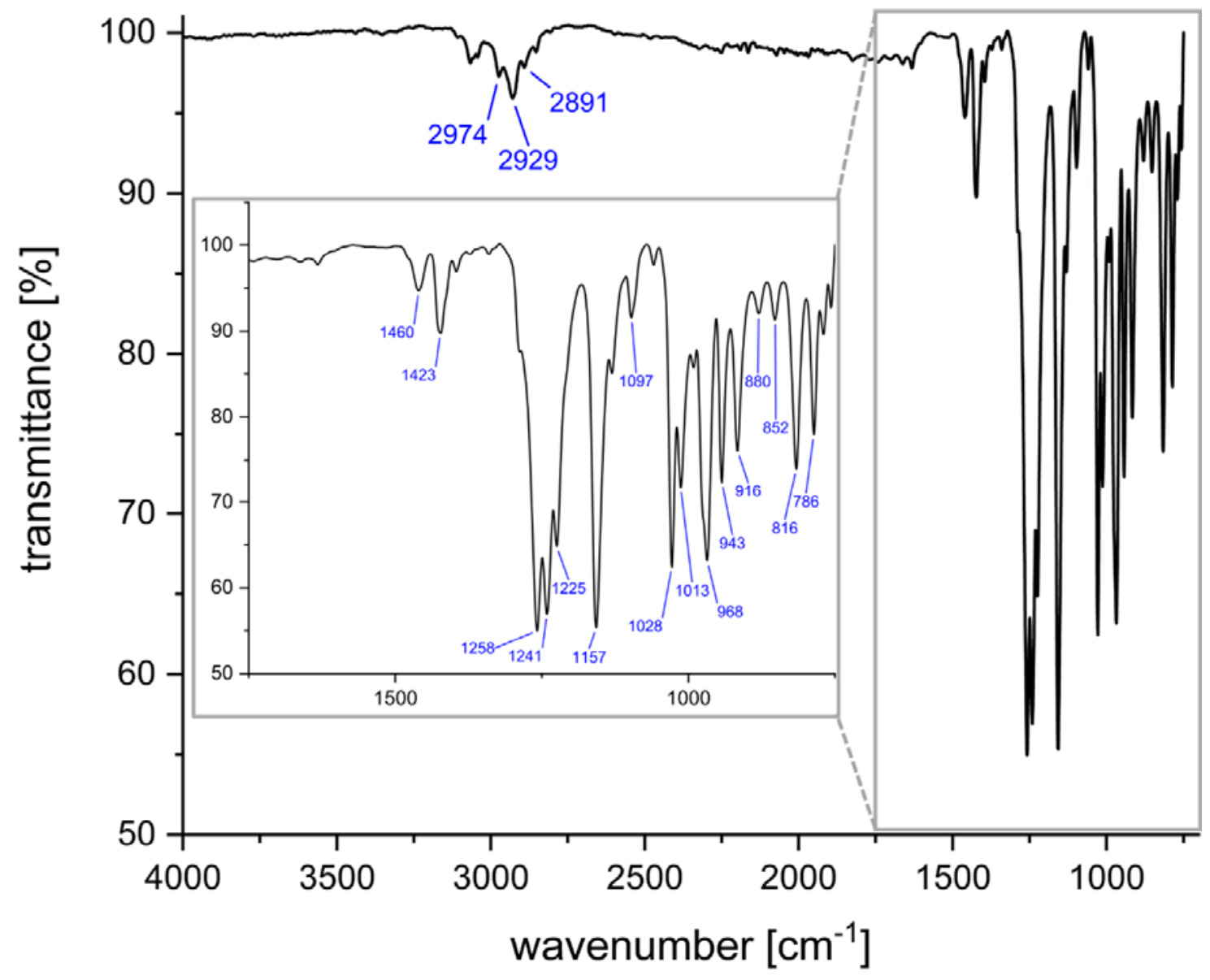

Figure S50. IR spectrum of $\left[\mathrm{Re}\left(\eta^{5}-\mathrm{C}_{5} \mathrm{H}_{4} \mathrm{CH}_{2} \mathrm{PTA}\right)\left(\eta^{6}-\mathrm{C}_{6} \mathrm{H}_{6}\right)\right](\mathrm{OTf})([11](\mathrm{OTf}))$ (neat). 


\section{Crystallographic Data}

Single crystal X-ray diffraction data were collected on a Rigaku OD Synergy (Pilatus 200K detector) diffractometer for $[3]\left(\mathrm{PF}_{6}\right), 8$ and 10, and on a Rigaku OD Xcalibur (Ruby CCD detector) for $\mathbf{4}$ and 6, both equipped with an Oxford liquid-nitrogen Cryostream cooler using a single wavelength $\mathrm{X}$-ray source from a micro-focus sealed $\mathrm{X}$-ray tube $(\mathrm{Cu} \mathrm{K} \alpha$ radiation $(\lambda=$ $1.54184 \AA)$ for $[3]\left(\mathrm{PF}_{6}\right)$ and 8 or Mo $\mathrm{K}_{\alpha}$ radiation $(\lambda=0.71073 \AA)$ for $\mathbf{4}, \mathbf{6}$ and 10). Suitable single crystals were manipulated into polybutene oil, selected and mounted on a flexible loop fixed on a goniometer head and transferred to the diffractometer. Pre-experiments, data collections, data reductions and analytical absorption corrections ${ }^{3}$ were performed with the program suite CrysAlis ${ }^{\text {Pro }}{ }^{4}$ Using Olex $2,{ }^{5}$ the structures were solved with the SHELXT small molecule structure solution program ${ }^{6}$ and refined with the SHELXL 2018/3 program package $^{7}$ by fullmatrix least-squares minimization on $\mathrm{F}^{2}$. PLATON was used to check the result of the X-ray analysis. ${ }^{8}$ The crystal data collections and structure refinement parameters are summarized in Tables S3, S6 and S8. CCDC 1993210 (for 4), 1993211 (for 10), 1993212 (for 8), 1993213 (for [3] $\left(\mathrm{PF}_{6}\right)$ ) and 1993214 (for 6) contain the supplementary crystallographic data for these compounds, and can be obtained free of charge from the Cambridge Crystallographic Data Centre via www.ccdc.cam.ac.uk/data request/cif.

In the crystal structure of $[3]\left(\mathrm{PF}_{6}\right)$, there are two half $\mathrm{C}_{12} \mathrm{H}_{12} \mathrm{Re}^{+}$cations in the asymmetric unit located on special positions and one $\mathrm{PF}_{6}$ counterion on a general position. Both cations are seriously disordered and many SHELXL restraints were used to correct the geometry of the disordered parts and the thermal parameters of the corresponding atoms. In the crystal structure of $\mathbf{8}$, the terminal methyl group is disordered over two sets of positions with site-occupancy factors refined to $0.33(3)$ and $0.67(3)$. In the crystal structure of 10, there are two crystallographically independent molecules in the asymmetric unit. For both molecules the terminal $\mathrm{CH}_{2}-\mathrm{CH}_{2}-\mathrm{OH}$ group is disordered over two sets of positions with site-occupancy factors of $0.401(11) / 0.599(11)$ and $0.428(15) / 0.572(15)$. 


\section{$4.1\left[\operatorname{Re}\left(\eta^{6}-\mathrm{C}_{5} \mathrm{H}_{4} \mathrm{CH}_{2}\right)\left(\eta^{6}-\mathrm{C}_{6} \mathrm{H}_{6}\right)\right]\left(\mathrm{PF}_{6}\right)\left([3]\left(\mathrm{PF}_{6}\right)\right)$}
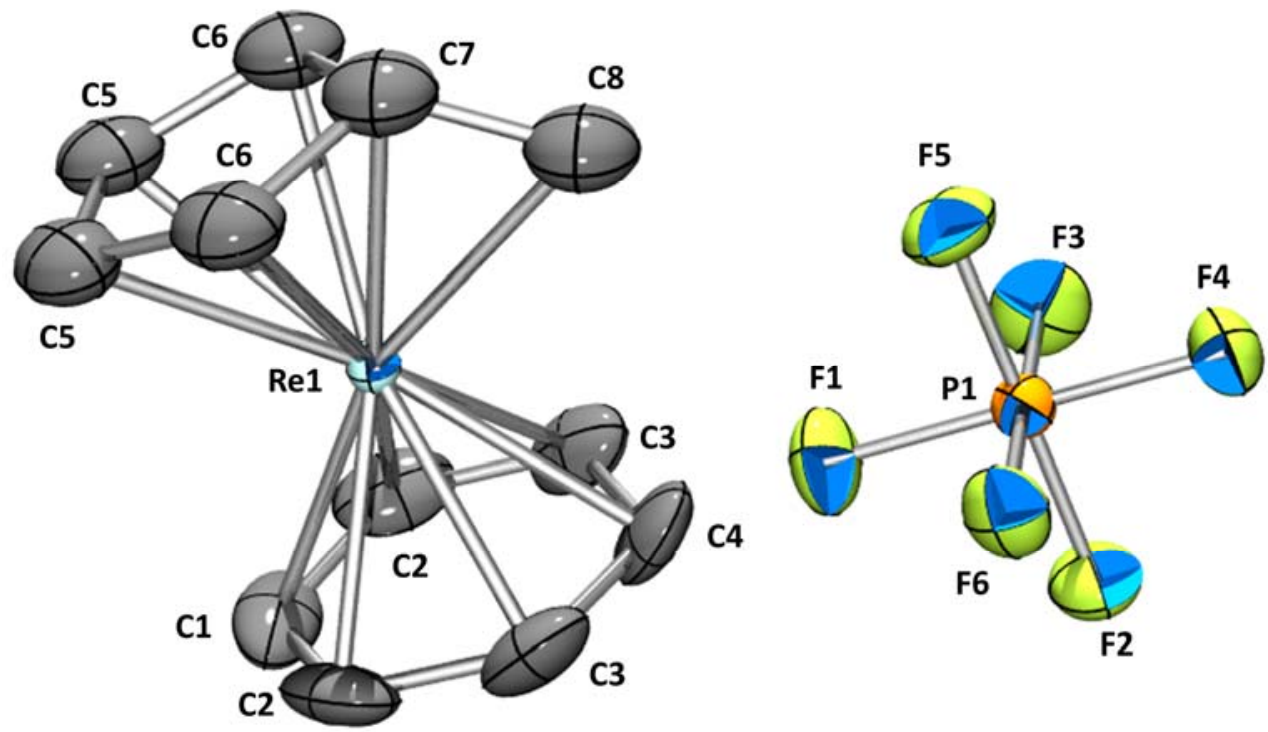

Figure S51. ORTEP representation of the optimized structure of $\left[\mathrm{Re}\left(\eta^{6}-\mathrm{C}_{5} \mathrm{H}_{4} \mathrm{CH}_{2}\right)\left(\eta^{6}-\mathrm{C}_{6} \mathrm{H}_{6}\right)\right]\left(\mathrm{PF}_{6}\right)\left([3]\left(\mathrm{PF}_{6}\right)\right)$. Hydrogen atoms have been omitted for clarity; thermal ellipsoids represent $50 \%$ probability.

Table S1. Bond Lengths for $\left[\mathrm{Re}\left(\eta^{6}-\mathrm{C}_{5} \mathrm{H}_{4} \mathrm{CH}_{2}\right)\left(\eta^{6}-\mathrm{C}_{6} \mathrm{H}_{6}\right)\right]\left(\mathrm{PF}_{6}\right)\left([3]\left(\mathrm{PF}_{6}\right)\right)$.

\begin{tabular}{lll|lll} 
Atom & Atom & Length $/ \AA$ & Atom & Atom & Length/ $\AA$ \\
\hline $\mathrm{C} 1$ & $\mathrm{C} 2$ & $1.412(7)$ & $\mathrm{C} 5$ & $\mathrm{C} 6$ & $1.402(8)$ \\
$\mathrm{C} 1$ & $\mathrm{Re} 1$ & $2.267(8)$ & $\mathrm{C} 5$ & $\mathrm{Re} 1$ & $2.281(6)$ \\
$\mathrm{C} 2$ & $\mathrm{C} 3$ & $1.398(8)$ & $\mathrm{C} 6$ & $\mathrm{C} 7$ & $1.470(7)$ \\
$\mathrm{C} 2$ & $\mathrm{Re} 1$ & $2.217(5)$ & $\mathrm{C} 6$ & $\mathrm{Re} 1$ & $2.209(5)$ \\
$\mathrm{C} 3$ & $\mathrm{C} 4$ & $1.403(7)$ & $\mathrm{C} 7$ & $\mathrm{C} 8$ & $1.351(11)$ \\
$\mathrm{C} 3$ & $\mathrm{Re} 1$ & $2.229(5)$ & $\mathrm{C} 7$ & $\mathrm{Re} 1$ & $2.105(7)$ \\
$\mathrm{C} 4$ & $\mathrm{Re} 1$ & $2.256(7)$ & $\mathrm{C} 8$ & $\mathrm{Re} 1$ & $2.267(9)$ \\
$\mathrm{C} 5$ & $\mathrm{C} 51$ & $1.400(11)$ & & &
\end{tabular}

${ }^{1}+\mathrm{X}, 1 / 2-\mathrm{Y},+\mathrm{Z}$ 


\section{$4.2\left[\operatorname{Re}\left(\eta^{5}-\mathrm{C}_{5} \mathrm{H}_{4} \mathrm{CH}=\mathrm{NCH}_{2} \mathrm{C}_{6} \mathrm{H}_{5}\right)\left(\eta^{6}-\mathrm{C}_{6} \mathrm{H}_{6}\right)\right](4)$}

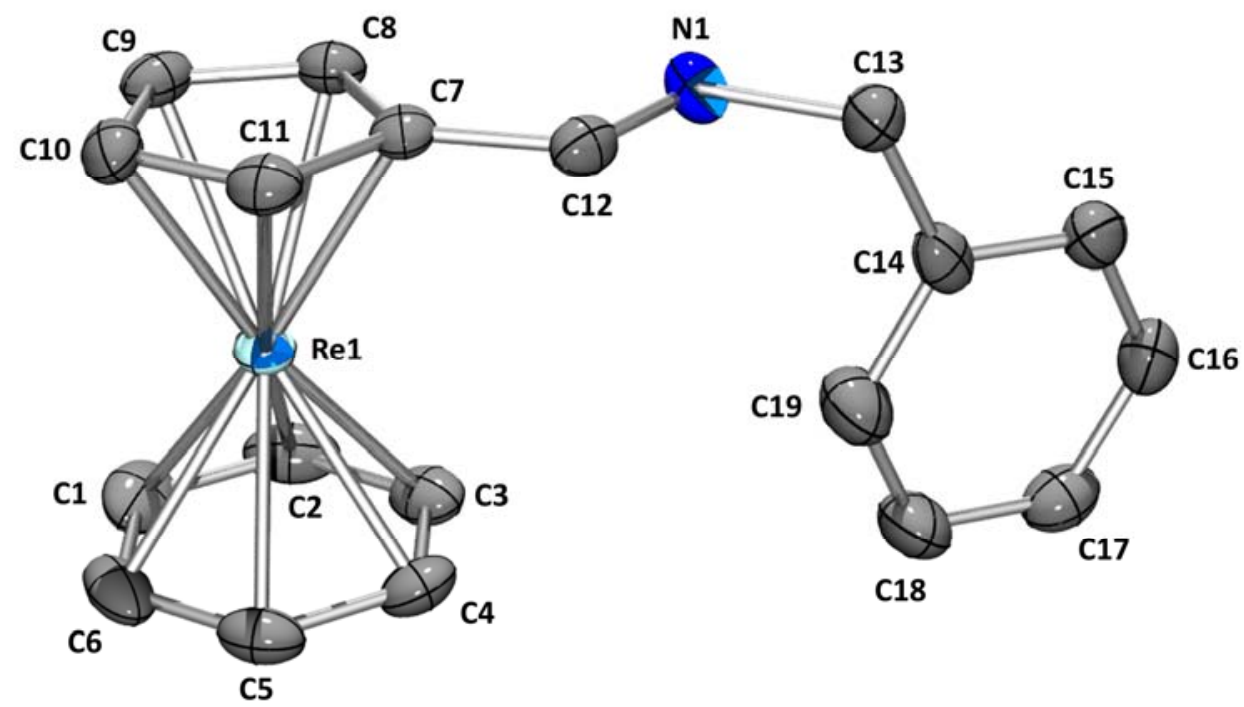

Figure S52. ORTEP representation of the optimized structure of $\left[\mathrm{Re}\left(\eta^{5}-\mathrm{C}_{5} \mathrm{H}_{4} \mathrm{CH}=\mathrm{NCH}_{2} \mathrm{C}_{6} \mathrm{H}_{5}\right)\left(\eta^{6}-\mathrm{C}_{6} \mathrm{H}_{6}\right)\right](4)$. Hydrogen atoms have been omitted for clarity; thermal ellipsoids represent $50 \%$ probability.

Table S2. Bond Lengths for $\left[\mathrm{Re}\left(\eta^{5}-\mathrm{C}_{5} \mathrm{H}_{4} \mathrm{CH}=\mathrm{NCH}_{2} \mathrm{C}_{6} \mathrm{H}_{5}\right)\left(\eta^{6}-\mathrm{C}_{6} \mathrm{H}_{6}\right)\right]$ (4).

\begin{tabular}{|c|c|c|c|c|c|}
\hline Atom & Atom & Length/Å & Atom & Atom & Length $/ \AA ̊$ \\
\hline $\operatorname{Re} 1$ & $\mathrm{C} 1$ & $2.206(7)$ & $\mathrm{C} 3$ & $\mathrm{C} 4$ & $1.409(8)$ \\
\hline $\operatorname{Re} 1$ & $\mathrm{C} 2$ & $2.208(6)$ & $\mathrm{C} 4$ & $\mathrm{C} 5$ & $1.40(1)$ \\
\hline $\operatorname{Re} 1$ & $\mathrm{C} 3$ & $2.195(6)$ & $\mathrm{C} 5$ & C6 & $1.40(1)$ \\
\hline Re1 & $\mathrm{C} 4$ & $2.203(6)$ & $\mathrm{C} 7$ & $\mathrm{C} 8$ & $1.449(7)$ \\
\hline $\operatorname{Re} 1$ & $\mathrm{C} 5$ & $2.204(5)$ & $\mathrm{C} 7$ & $\mathrm{C} 11$ & $1.431(8)$ \\
\hline $\operatorname{Re} 1$ & $\mathrm{C} 6$ & $2.219(6)$ & $\mathrm{C} 7$ & $\mathrm{C} 12$ & $1.454(8)$ \\
\hline $\operatorname{Re} 1$ & $\mathrm{C} 7$ & $2.236(6)$ & $\mathrm{C} 8$ & $\mathrm{C} 9$ & $1.420(9)$ \\
\hline $\operatorname{Re} 1$ & $\mathrm{C} 8$ & $2.231(5)$ & C9 & $\mathrm{C} 10$ & $1.424(8)$ \\
\hline $\operatorname{Re} 1$ & C9 & $2.236(5)$ & $\mathrm{C} 10$ & $\mathrm{C} 11$ & $1.407(8)$ \\
\hline $\operatorname{Re} 1$ & $\mathrm{C} 10$ & $2.247(6)$ & $\mathrm{C} 13$ & $\mathrm{C} 14$ & $1.517(8)$ \\
\hline $\operatorname{Re} 1$ & $\mathrm{C} 11$ & $2.264(5)$ & $\mathrm{C} 14$ & $\mathrm{C} 15$ & $1.401(9)$ \\
\hline $\mathrm{N} 1$ & $\mathrm{C} 12$ & $1.292(8)$ & $\mathrm{C} 14$ & $\mathrm{C} 19$ & $1.373(9)$ \\
\hline $\mathrm{N} 1$ & $\mathrm{C} 13$ & $1.464(8)$ & $\mathrm{C} 15$ & $\mathrm{C} 16$ & $1.392(9)$ \\
\hline $\mathrm{C} 1$ & $\mathrm{C} 2$ & $1.41(1)$ & $\mathrm{C} 16$ & $\mathrm{C} 17$ & $1.37(1)$ \\
\hline $\mathrm{C} 1$ & $\mathrm{C} 6$ & $1.43(1)$ & $\mathrm{C} 17$ & $\mathrm{C} 18$ & $1.39(1)$ \\
\hline $\mathrm{C} 2$ & $\mathrm{C} 3$ & $1.39(1)$ & $\mathrm{C} 18$ & $\mathrm{C} 19$ & $1.38(1)$ \\
\hline
\end{tabular}


Table S3. Crystal data and structure refinement parameters for $[3]\left(\mathrm{PF}_{6}\right)$ and 4.

\begin{tabular}{|c|c|c|}
\hline & {$[3]\left(\mathrm{PF}_{6}\right)$} & 4 \\
\hline CCDC number & 1993213 & 1993210 \\
\hline Empirical formula & $\mathrm{C}_{12} \mathrm{H}_{12} \mathrm{~F}_{6} \mathrm{PRe}$ & $\mathrm{C}_{19} \mathrm{H}_{18} \mathrm{NRe}$ \\
\hline Formula weight & 487.39 & 446.54 \\
\hline Temperature/K & $183(1)$ & $183(1)$ \\
\hline Crystal system & orthorhombic & triclinic \\
\hline Space group & Pnma & $\mathrm{P}-1$ \\
\hline $\mathrm{a} / \AA$ & $9.63900(9)$ & $5.9120(3)$ \\
\hline $\mathrm{b} / \AA$ & $27.0212(3)$ & $9.5757(6)$ \\
\hline $\mathrm{c} / \AA$ & $9.95098(11)$ & $13.3629(6)$ \\
\hline$\alpha /{ }^{\circ}$ & 90 & $100.995(4)$ \\
\hline$\beta /{ }^{\circ}$ & 90 & $90.737(4)$ \\
\hline$\gamma /{ }^{\circ}$ & 90 & $98.449(4)$ \\
\hline Volume $/ \AA^{3}$ & $2591.81(4)$ & $733.90(7)$ \\
\hline $\mathrm{Z}$ & 8 & 2 \\
\hline$\rho_{\text {calc }} \mathrm{g} / \mathrm{cm}^{3}$ & 2.498 & 2.021 \\
\hline$\mu / \mathrm{mm}^{-1}$ & 20.111 & 8.271 \\
\hline $\mathrm{F}(000)$ & 1824.0 & 428.0 \\
\hline Crystal size $/ \mathrm{mm}^{3}$ & $0.20 \times 0.04 \times 0.03$ & $0.14 \times 0.12 \times 0.01$ \\
\hline Radiation & $\operatorname{CuK} \alpha(\lambda=1.54184)$ & $\operatorname{Mo} \operatorname{K} \alpha(\lambda=0.71073)$ \\
\hline $2 \Theta$ range for data collection/ ${ }^{\circ}$ & 6.542 to 136.398 & 4.384 to 61.016 \\
\hline Index ranges & $-11 \leq \mathrm{h} \leq 11,-32 \leq \mathrm{k} \leq 32,-11 \leq 1 \leq 11$ & $-7 \leq \mathrm{h} \leq 8,-13 \leq \mathrm{k} \leq 12,-19 \leq 1 \leq 13$ \\
\hline Reflections collected & 29417 & 8973 \\
\hline Independent reflections & $2423\left[\mathrm{R}_{\mathrm{int}}=0.0374, \mathrm{R}_{\mathrm{sigma}}=0.0121\right]$ & $4473\left[\mathrm{R}_{\text {int }}=0.0517, \mathrm{R}_{\text {sigma }}=0.0723\right]$ \\
\hline Data/restraints/parameters & $2423 / 272 / 299$ & $4473 / 0 / 190$ \\
\hline Goodness-of-fit on $\mathrm{F}^{2}$ & 1.077 & 1.041 \\
\hline Final $R$ indexes $[\mathrm{I}>=2 \sigma(\mathrm{I})]$ & $\mathrm{R}_{1}=0.0245, \mathrm{wR}_{2}=0.0643$ & $\mathrm{R}_{1}=0.0435, \mathrm{wR}_{2}=0.0822$ \\
\hline Final $\mathrm{R}$ indexes [all data] & $\mathrm{R}_{1}=0.0247, \mathrm{wR}_{2}=0.0645$ & $\mathrm{R}_{1}=0.0531, \mathrm{wR}_{2}=0.0864$ \\
\hline Largest diff. peak/hole / e $\AA^{-3}$ & $2.57 /-1.87$ & $2.78 /-1.50$ \\
\hline
\end{tabular}




\section{$4.3\left[\operatorname{Re}\left(\eta^{5}-\mathrm{C}_{5} \mathrm{H}_{4} \mathrm{CH}_{2} \mathrm{CN}\right)\left(\eta^{6}-\mathrm{C}_{6} \mathrm{H}_{6}\right)\right](6)$}

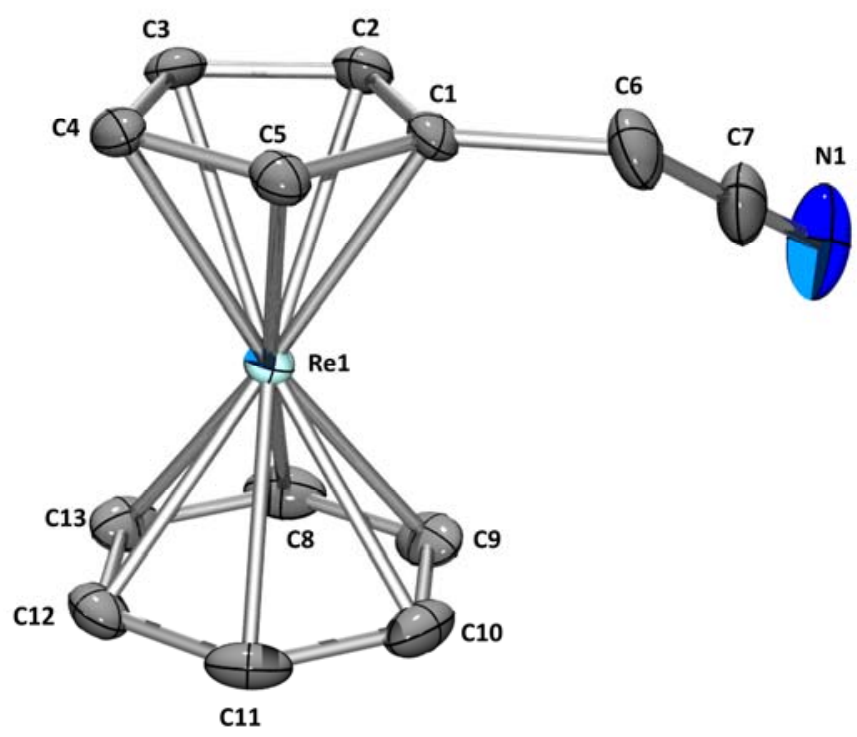

Figure S53. ORTEP representation of the optimized structure of $\left[\mathrm{Re}\left(\eta^{5}-\mathrm{C}_{5} \mathrm{H}_{4} \mathrm{CH}_{2} \mathrm{CN}\right)\left(\eta^{6}-\mathrm{C}_{6} \mathrm{H}_{6}\right)\right](6)$. Hydrogen atoms have been omitted for clarity; thermal ellipsoids represent $50 \%$ probability.

Table S4. Bond Lengths for $\left[\mathrm{Re}\left(\eta^{5}-\mathrm{C}_{5} \mathrm{H}_{4} \mathrm{CH}_{2} \mathrm{CN}\right)\left(\eta^{6}-\mathrm{C}_{6} \mathrm{H}_{6}\right)\right](6)$.

\begin{tabular}{|c|c|c|c|c|c|}
\hline Atom & Atom & Length/Å & Atom & Atom & Length/Å \\
\hline Re1 & $\mathrm{C} 1$ & $2.238(4)$ & $\mathrm{C} 1$ & $\mathrm{C} 5$ & $1.419(5)$ \\
\hline Re1 & $\mathrm{C} 2$ & $2.242(4)$ & $\mathrm{C} 1$ & C6 & $1.509(5)$ \\
\hline Re1 & $\mathrm{C} 3$ & $2.246(4)$ & $\mathrm{C} 2$ & $\mathrm{C} 3$ & $1.427(5)$ \\
\hline Re1 & $\mathrm{C} 4$ & $2.251(4)$ & $\mathrm{C} 3$ & $\mathrm{C} 4$ & $1.421(6)$ \\
\hline Re1 & $\mathrm{C} 5$ & $2.241(4)$ & $\mathrm{C} 4$ & $\mathrm{C} 5$ & $1.418(6)$ \\
\hline Re1 & $\mathrm{C} 8$ & $2.211(4)$ & C6 & $\mathrm{C} 7$ & $1.466(6)$ \\
\hline Re1 & $\mathrm{C} 9$ & $2.206(4)$ & $\mathrm{C} 8$ & C9 & $1.402(6)$ \\
\hline Re1 & $\mathrm{C} 10$ & $2.205(4)$ & $\mathrm{C} 8$ & $\mathrm{C} 13$ & $1.408(6)$ \\
\hline Re1 & C11 & $2.197(4)$ & C9 & $\mathrm{C} 10$ & $1.427(6)$ \\
\hline Re1 & C12 & $2.204(4)$ & $\mathrm{C} 10$ & $\mathrm{C} 11$ & $1.415(6)$ \\
\hline Re1 & C13 & $2.208(4)$ & $\mathrm{C} 11$ & $\mathrm{C} 12$ & $1.411(6)$ \\
\hline N1 & $\mathrm{C} 7$ & $1.136(6)$ & $\mathrm{C} 12$ & $\mathrm{C} 13$ & $1.417(6)$ \\
\hline $\mathrm{C} 1$ & $\mathrm{C} 2$ & $1.429(5)$ & & & \\
\hline
\end{tabular}




\section{$4.4\left[\operatorname{Re}\left(\eta^{5}-\mathrm{C}_{5} \mathrm{H}_{4} \mathrm{CH}_{2} \mathrm{OMe}\right)\left(\eta^{6}-\mathrm{C}_{6} \mathrm{H}_{6}\right)\right](8)$}

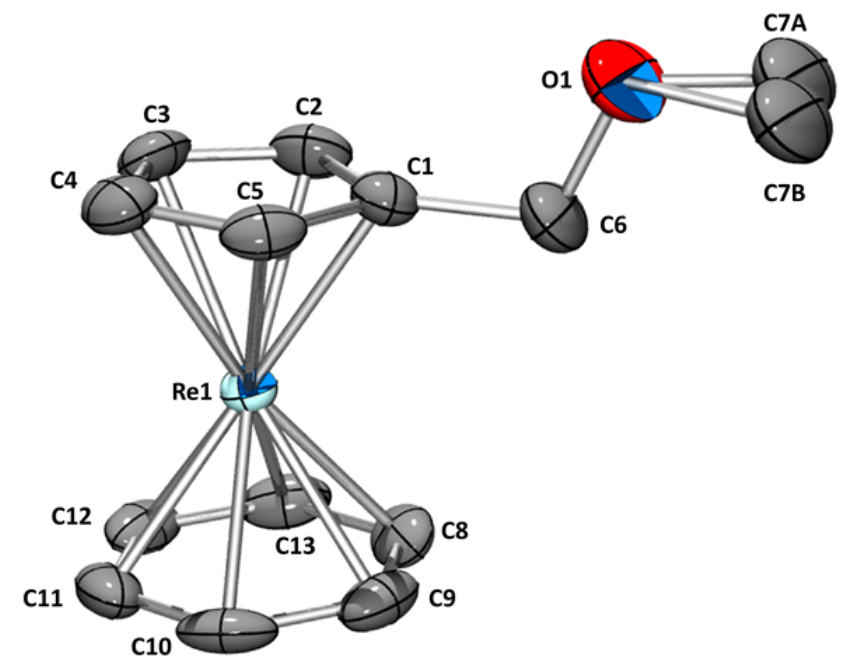

Figure S54. ORTEP representation of the optimized structure of $\left[\mathrm{Re}\left(\eta^{5}-\mathrm{C}_{5} \mathrm{H}_{4} \mathrm{CH}_{2} \mathrm{OMe}\right)\left(\eta^{6}-\mathrm{C}_{6} \mathrm{H}_{6}\right)\right](8)$. Hydrogen atoms have been omitted for clarity; thermal ellipsoids represent $50 \%$ probability.

\section{Special features}

The terminal $\mathrm{CH}_{3}$ group is disordered over two sets of positions with site-occupancy factors refined to $0.33(3)$ for C7A and 0.67(3) for C7B.

Table S5. Bond lengths for $\left[\mathrm{Re}\left(\eta^{5}-\mathrm{C}_{5} \mathrm{H}_{4} \mathrm{CH}_{2} \mathrm{OMe}\right)\left(\eta^{6}-\mathrm{C}_{6} \mathrm{H}_{6}\right)\right](8)$.

\begin{tabular}{|c|c|c|c|c|c|}
\hline Atom & Atom & Length/Å & Atom & Atom & Length/Å \\
\hline Re1 & $\mathrm{C} 1$ & $2.216(4)$ & $\mathrm{O} 1$ & $\mathrm{C} 7 \mathrm{~B}$ & $1.460(9)$ \\
\hline Re1 & $\mathrm{C} 2$ & $2.233(4)$ & $\mathrm{C} 1$ & $\mathrm{C} 2$ & $1.433(7)$ \\
\hline Re1 & $\mathrm{C} 3$ & $2.254(4)$ & $\mathrm{C} 1$ & $\mathrm{C} 5$ & $1.430(7)$ \\
\hline Re1 & $\mathrm{C} 4$ & $2.250(4)$ & $\mathrm{C} 1$ & C6 & $1.479(7)$ \\
\hline Re1 & $\mathrm{C} 5$ & $2.237(4)$ & $\mathrm{C} 2$ & $\mathrm{C} 3$ & $1.411(7)$ \\
\hline Re1 & $\mathrm{C} 8$ & $2.213(4)$ & $\mathrm{C} 3$ & $\mathrm{C} 4$ & $1.409(7)$ \\
\hline Re1 & C9 & $2.213(5)$ & $\mathrm{C} 4$ & $\mathrm{C} 5$ & $1.413(7)$ \\
\hline Re1 & $\mathrm{C} 10$ & $2.219(4)$ & $\mathrm{C} 8$ & C9 & $1.415(7)$ \\
\hline Re1 & $\mathrm{C} 11$ & $2.207(5)$ & $\mathrm{C} 8$ & $\mathrm{C} 13$ & $1.412(7)$ \\
\hline Re1 & $\mathrm{C} 12$ & $2.209(4)$ & C9 & $\mathrm{C} 10$ & $1.414(8)$ \\
\hline Re1 & $\mathrm{C} 13$ & $2.210(4)$ & $\mathrm{C} 10$ & $\mathrm{C} 11$ & $1.411(8)$ \\
\hline $\mathrm{O} 1$ & C6 & $1.414(6)$ & $\mathrm{C} 11$ & $\mathrm{C} 12$ & $1.403(8)$ \\
\hline $\mathrm{O} 1$ & C7A & $1.463(10)$ & $\mathrm{C} 12$ & $\mathrm{C} 13$ & $1.405(8)$ \\
\hline
\end{tabular}


Table S6. Crystal data and structure refinement parameters for $\mathbf{6}$ and $\mathbf{8}$.

\begin{tabular}{|c|c|c|}
\hline & 6 & 8 \\
\hline CCDC number & 1993214 & 1993212 \\
\hline Empirical formula & $\mathrm{C}_{13} \mathrm{H}_{12} \mathrm{NRe}$ & $\mathrm{C}_{13} \mathrm{H}_{15} \mathrm{ORe}$ \\
\hline Formula weight & 368.44 & 373.45 \\
\hline Temperature/K & $159.8(10)$ & $160(1)$ \\
\hline Crystal system & orthorhombic & trigonal \\
\hline Space group & $\mathrm{P} 2{ }_{1} 2_{1} 2_{1}$ & $\mathrm{R}-3$ \\
\hline $\mathrm{a} / \AA$ & $5.88427(18)$ & $30.9417(7)$ \\
\hline $\mathrm{b} / \AA$ & $9.9756(3)$ & $30.9417(7)$ \\
\hline $\mathrm{c} / \AA$ & $18.1013(6)$ & $5.9990(2)$ \\
\hline$\alpha /^{\circ}$ & 90 & 90 \\
\hline$\beta /{ }^{\circ}$ & 90 & 90 \\
\hline$\gamma /{ }^{\circ}$ & 90 & 120 \\
\hline Volume $/ \AA^{3}$ & $1062.53(6)$ & $4973.9(3)$ \\
\hline Z & 4 & 18 \\
\hline$\rho_{\text {calc }} \mathrm{g} / \mathrm{cm}^{3}$ & 2.303 & 2.244 \\
\hline$\mu / \mathrm{mm}^{-1}$ & 11.398 & 21.237 \\
\hline $\mathrm{F}(000)$ & 688.0 & 3168.0 \\
\hline Crystal size $/ \mathrm{mm}^{3}$ & $0.23 \times 0.11 \times 0.08$ & $0.11 \times 0.04 \times 0.02$ \\
\hline Radiation & $\operatorname{MoK} \alpha(\lambda=0.71073)$ & $\mathrm{CuK} \alpha(\lambda=1.54184)$ \\
\hline $2 \Theta$ range for data collection ${ }^{\circ}$ & 6.078 to 60.98 & 9.902 to 148.472 \\
\hline Index ranges & $-8 \leq \mathrm{h} \leq 8,-14 \leq \mathrm{k} \leq 14,-25 \leq 1 \leq 25$ & $-38 \leq \mathrm{h} \leq 28,-30 \leq \mathrm{k} \leq 38,-7 \leq 1 \leq 7$ \\
\hline Reflections collected & 16076 & 9167 \\
\hline Independent reflections & $3246\left[\mathrm{R}_{\text {int }}=0.0330, \mathrm{R}_{\text {sigma }}=0.0248\right]$ & $2255\left[\mathrm{R}_{\text {int }}=0.0363, \mathrm{R}_{\text {sigma }}=0.0300\right]$ \\
\hline Data/restraints/parameters & $3246 / 0 / 136$ & $2255 / 19 / 148$ \\
\hline Goodness-of-fit on $\mathrm{F}^{2}$ & 1.018 & 1.060 \\
\hline Final $R$ indexes $[\mathrm{I}>=2 \sigma(\mathrm{I})]$ & $\mathrm{R}_{1}=0.0154, \mathrm{wR}_{2}=0.0318$ & $\mathrm{R}_{1}=0.0230, \mathrm{wR}_{2}=0.0557$ \\
\hline Final $\mathrm{R}$ indexes [all data] & $\mathrm{R}_{1}=0.0164, \mathrm{wR}_{2}=0.0322$ & $\mathrm{R}_{1}=0.0275, \mathrm{wR}_{2}=0.0571$ \\
\hline Largest diff. peak/hole / e $\AA^{-3}$ & $0.57 /-0.58$ & $0.51 /-1.08$ \\
\hline Flack parameter & $-0.024(8)$ & - \\
\hline
\end{tabular}




\section{$4.5\left[\operatorname{Re}\left(\eta^{5}-\mathrm{C}_{5} \mathrm{H}_{4} \mathrm{CH}_{2} \mathrm{~S}\left(\mathrm{CH}_{2}\right)_{2} \mathrm{OH}\right)\left(\eta^{6}-\mathrm{C}_{6} \mathrm{H}_{6}\right)\right](10)$}

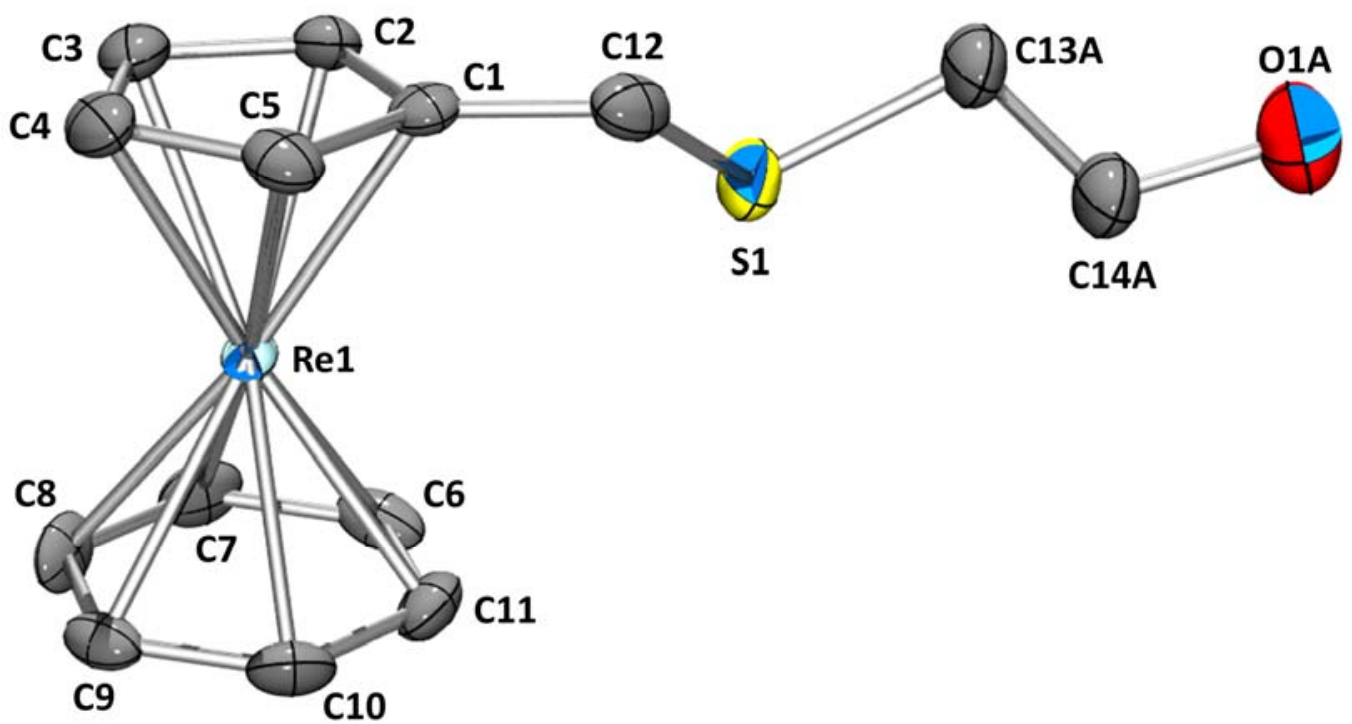

Figure S55. ORTEP representation of the optimized structure of $\left[\mathrm{Re}\left(\eta^{5}-\mathrm{C}_{5} \mathrm{H}_{4} \mathrm{CH}_{2} \mathrm{~S}\left(\mathrm{CH}_{2}\right)_{2} \mathrm{OH}\right)\left(\eta^{6}-\mathrm{C}_{6} \mathrm{H}_{6}\right)\right](\mathbf{1 0})$. Hydrogen atoms, the second crystallographically independent molecule and disorders have been omitted for clarity; thermal ellipsoids represent $50 \%$ probability.

Table S7. Bond lengths for $\left[\mathrm{Re}\left(\eta^{5}-\mathrm{C}_{5} \mathrm{H}_{4} \mathrm{CH}_{2} \mathrm{~S}\left(\mathrm{CH}_{2}\right)_{2} \mathrm{OH}\right)\left(\eta^{6}-\mathrm{C}_{6} \mathrm{H}_{6}\right)\right](\mathbf{1 0})$.

\begin{tabular}{|c|c|c|c|c|c|}
\hline Atom & Atom & Length/Å & Atom & Atom & Length/Å \\
\hline $\mathrm{C} 1$ & $\mathrm{C} 2$ & $1.43(1)$ & $\mathrm{C} 7$ & $\mathrm{C} 8$ & $1.41(1)$ \\
\hline $\mathrm{C} 1$ & $\mathrm{C} 5$ & $1.44(1)$ & $\mathrm{C} 7$ & Re1 & $2.209(9)$ \\
\hline $\mathrm{C} 1$ & $\mathrm{C} 12$ & $1.48(1)$ & $\mathrm{C} 8$ & C9 & $1.41(1)$ \\
\hline $\mathrm{C} 1$ & Re1 & $2.261(8)$ & $\mathrm{C} 8$ & Re1 & $2.214(8)$ \\
\hline $\mathrm{C} 2$ & $\mathrm{C} 3$ & $1.43(1)$ & C9 & $\mathrm{C} 10$ & $1.42(1)$ \\
\hline $\mathrm{C} 2$ & Re1 & $2.254(8)$ & C9 & Re1 & $2.221(9)$ \\
\hline $\mathrm{C} 3$ & $\mathrm{C} 4$ & $1.40(1)$ & $\mathrm{C} 10$ & $\mathrm{C} 11$ & $1.42(1)$ \\
\hline $\mathrm{C} 3$ & Re1 & $2.248(8)$ & $\mathrm{C} 10$ & Re1 & $2.211(9)$ \\
\hline $\mathrm{C} 4$ & $\mathrm{C} 5$ & $1.44(1)$ & $\mathrm{C} 11$ & Re1 & $2.207(8)$ \\
\hline $\mathrm{C} 4$ & Re1 & $2.249(9)$ & $\mathrm{C} 12$ & $\mathrm{~S} 1$ & $1.79(1)$ \\
\hline $\mathrm{C} 5$ & Re1 & $2.252(9)$ & $\mathrm{C} 13 \mathrm{~A}$ & C14A & $1.49(2)$ \\
\hline C6 & $\mathrm{C} 7$ & $1.43(1)$ & $\mathrm{C} 13 \mathrm{~A}$ & $\mathrm{~S} 1$ & $1.83(1)$ \\
\hline C6 & C11 & $1.42(1)$ & $\mathrm{C} 14 \mathrm{~A}$ & $\mathrm{O} 1 \mathrm{~A}$ & $1.49(2)$ \\
\hline C6 & Re1 & $2.210(8)$ & & & \\
\hline
\end{tabular}


Table S8. Crystal data and structure refinement parameters for $\mathbf{1 0}$.

\begin{tabular}{|c|c|}
\hline & 10 \\
\hline CCDC number & 1993211 \\
\hline Empirical formula & $\mathrm{C}_{14} \mathrm{H}_{17} \mathrm{OReS}$ \\
\hline Formula weight & 419.53 \\
\hline Temperature/K & $183(1)$ \\
\hline Crystal system & tetragonal \\
\hline Space group & $\mathrm{P} 4_{2}$ \\
\hline $\mathrm{a} / \AA$ & $20.7788(4)$ \\
\hline $\mathrm{b} / \AA$ & $20.7788(4)$ \\
\hline $\mathrm{c} / \AA ̊$ & $5.9803(2)$ \\
\hline$\alpha /{ }^{\circ}$ & 90 \\
\hline$\beta /^{\circ}$ & 90 \\
\hline$\gamma /{ }^{\circ}$ & 90 \\
\hline Volume $/ \AA^{3}$ & $2582.05(13)$ \\
\hline Z & 8 \\
\hline$\rho_{\text {calc }} \mathrm{g} / \mathrm{cm}^{3}$ & 2.158 \\
\hline$\mu / \mathrm{mm}^{-1}$ & 9.554 \\
\hline $\mathrm{F}(000)$ & 1600.0 \\
\hline Crystal size $/ \mathrm{mm}^{3}$ & $0.29 \times 0.02 \times 0.015$ \\
\hline Radiation & $\operatorname{Mo} \operatorname{K\alpha }(\lambda=0.71073)$ \\
\hline $2 \Theta$ range for data collection ${ }^{\circ}$ & 5.546 to 61.012 \\
\hline Index ranges & $-20 \leq \mathrm{h} \leq 29,-29 \leq \mathrm{k} \leq 29,-8 \leq 1 \leq 8$ \\
\hline Reflections collected & 24643 \\
\hline Independent reflections & $7853\left[\mathrm{R}_{\text {int }}=0.0403, \mathrm{R}_{\text {sigma }}=0.0534\right]$ \\
\hline Data/restraints/parameters & $7853 / 390 / 358$ \\
\hline Goodness-of-fit on $\mathrm{F}^{2}$ & 1.028 \\
\hline Final $\mathrm{R}$ indexes $[\mathrm{I}>=2 \sigma(\mathrm{I})]$ & $\mathrm{R}_{1}=0.0335, \mathrm{wR}_{2}=0.0575$ \\
\hline Final $\mathrm{R}$ indexes [all data] & $\mathrm{R}_{1}=0.0447, \mathrm{wR}_{2}=0.0595$ \\
\hline Largest diff. peak/hole / e $\AA^{-3}$ & $1.68 /-1.34$ \\
\hline Flack parameter & $-0.019(8)$ \\
\hline
\end{tabular}




\section{References}

(1) Meola, G.; Braband, H.; Schmutz, P.; Benz, M.; Spingler, B.; Alberto, R. Bis-Arene Complexes $\left[\operatorname{Re}\left(\eta^{6}-\right.\right.$ arene) $]^{+}$as Highly Stable Bioorganometallic Scaffolds. Inorg. Chem. 2016, 55, 11131-11139.

(2) Meola, G.; Braband, H.; Hernández-Valdés, D.; Gotzmann, C.; Fox, T.; Spingler, B.; Alberto, R. A MixedRing Sandwich Complex from Unexpected Ring Contraction in $\left[\mathrm{Re}\left(\eta^{6}-\mathrm{C}_{6} \mathrm{H}_{5} \mathrm{Br}\right)\left(\eta^{6}-\mathrm{C}_{6} \mathrm{R}_{6}\right)\right]\left(\mathrm{PF}_{6}\right)$. Inorg. Chem. 2017, 56, 6297-6301.

(3) Clark, R. C.; Reid, J. S. The analytical calculation of absorption in multifaceted crystals. Acta Cryst. A 1995, 51, 887-897.

(4) Rigaku Oxford Diffraction. 2020.

(5) Dolomanov, O. V.; Bourhis, L. J.; Gildea, R. J.; Howard, J. A. K.; Puschmann, H. OLEX2: a complete structure solution, refinement and analysis program. J. Appl. Crystallogr. 2009, 42, 339-341.

(6) Sheldrick, G. M. SHELXT - Integrated space-group and crystal-structure determination. Acta Cryst. A 2015, $71,3-8$.

(7) Sheldrick, G. M. Crystal structure refinement with SHELXL. Acta Cryst. C 2015, 71, 3-8.

(8) Spek, A. L. PLATON SQUEEZE: a tool for the calculation of the disordered solvent contribution to the calculated structure factors Acta Cryst. C 2015, 71, 9-18. 